\title{
CASCADE OF NEGATIVE MUONS IN ATOMS
}

\author{
THESIS BY \\ VICTOR R. AKYLAS
}

In Partial Fulfillment of the Requirements

FOR THE DEgREe OF

DOCTOR OF PHILOSOPHY

California Institute of Technology
Pasadena, California

1978

(Submitted May 5,1978 ) 


\section{ACKNOWLEDGEMENTS}

The author would like to take this opportunity to express his gratitude and appreciation for those whose help and contributions made it all possible:

Professor Felix H. Boehm for his leadership, encouragement, and sincere interest,

Dr. Petr vogel for his endless assistance, productive criticism and advice, most of the time beyond the call of duty, and for his personal attention to all work,

Professor Aage winther for his unlimited help and sharp insight,

Drs. Jean-Luc Vuilleumier and Alan A. Hahn for their interesting discussions and helpful remarks,

Mrs. Elsa Garcia for her constant interest and help with typing and technical matters,

Ali members of the Nuclear Spectroscopy Group for their hospitality, contributions, and relaxing diversions,

The Physics Department for the education and financial assistance,

The Energy Research and Development Administration and the Department of Energy of the linited States for their financial support under contracts AT[04-3]-63 and EY-76-C-03-0063,

The Earle C. Antinony Fellowship Fund for a graduate jellowship,

The Booth Computing Center of the Institute for their facilities used for this thesis free of charge,

The California Institute of Technology for the research environment and numerous instances of assistance,

All colleagues and friends for their help and support. 
The subject of this thesis is the study of the evolution of a negative muon captured in an atom and the formalism of energy loss associated with the muonic atom. The principal goals are to calculate reliably the muon $x$-ray intensities, given the initial population of the muonic orbits, to invert the problem and deduce the initial distribution from the $x$-ray intensities, to provide the experimentalist with a reasonably simple and convenient tool to correlate his observations, and finally, to systematize some questions of theoretical interest. The early part of the history of the muon in matter, including the atomic capture and classical phase of the atomic cascade are reviewed. In the quantal treatment of the transition rates, both radiative and electron Auger transitions are considered. In general, multipolarities up to $E 3$ and $\mathrm{K}, \mathrm{L}$, and $\mathrm{M}$ electronic shells are fully investigated. Multipole radiation is treated in the conventional way and presents no special problems. Magnetic type transitions between states with different principal quantum numbers are shown to be small. Auger electron ejection rates are more complicated and several approximations have been adopted. The basic results have been computed in terms of elementary functions. The relativistic retardation effect is significant at high transition energies, where Auger rates are unimportant. Similarly, the effect of the electron screening of the muon has no significant influence on the results. The calculation of the penetration makes the transition rates reliable. In the Auger transitions we have shown that magnetic multipoles can be safely neglected. The relative sizes of the rates corresponding to different multipoles are systematically studied. The $E 1$ Auger rates are generally largest, but the $E 0$ and $E 2$ transitions are also substantial. Penetration usually decreases rates, being mostly important for transitions with small continuum electron momentum. A comparison of our results is made with atomic photoelectric effect data and with the nuclear internal conversion coefficients. A general agreement is found, except around shell thresholds. The existing data of muonic x-ray intensities in iron and thallium are analyzed in a systematic way. The data are fitted with an initial $Z$-distribution and some other derived physical parameters. The quality of the fits is good. It is found that for Fe the initial l-distribution is almost $\mathrm{flat}$, whereas that for $\mathrm{Tl}$ is weighted towards the high $\mathrm{l}$ values, sharper than statistical. As a result of the investigations and in order to make our findings usable, a computer program has been developed. This program is superior to the present standard one, including more precise calculation of transition rates, wider choice of parameters, and a flexible input/output section. 


\section{$-i v-$}

TABLE OF CONTENTS

ACKNOWLEDGEMENTS

ABSTRACT

ii i

TABLE OF CONTENTS

LIST OF FIGURES, TABLES, AND INPUT/OUTPUT PAGES

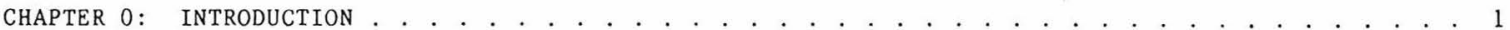

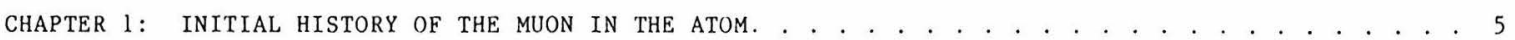

1.1 Energy Loss from the Continuum 5

1.1.1 Justification of the Classical Approach 5

1.1.2 Description of the Energy Loss Mechanisms 6

1.1 .3 Comments on the Calculation $\quad 8$

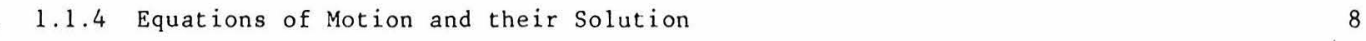

$\begin{array}{llr}1.1 .5 & \text { Cross Sections } & 9\end{array}$

1.2 Muon Capture from the Continuum 9

1.2.1 Angular Momentum at Capture 11

1.2.2 Compounds 11

1.3 Classical Part of the Cascade 13

1.4 Depolarization of the Muons $\quad 15$

1.4.1 Spin Deflection in Muon-Electron Collisions $\quad 15$

1.4.2 Depolarization Due to the Spin-Orbit Coupling 17

$\begin{array}{lll}\text { 1.4.3 Quantal Depolarization } & 17\end{array}$

$\begin{array}{llr}1.5 & \text { Semiclassical Approach } & 18\end{array}$

Chapter 2: Quantal Cascade and transition rates .......................... 21

$\begin{array}{ll}2.1 \text { Overview of the Quantal Mechanisms } & 21\end{array}$

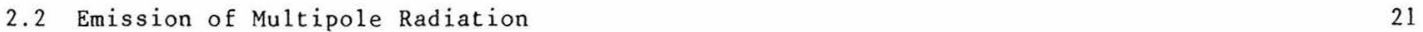

2.3 Energy Loss via Ejection of Atomic Electrons (Auger Transitions) 22

2.4 Improvements on the Transition Rates 24

$\begin{array}{llr}2.4 .1 & \text { Retardation } & 24\end{array}$

2.4.2 Electronic Screening $\quad 24$

$\begin{array}{llr}2.4 .3 & \text { Penetration } & 24\end{array}$

2.5 Relative Importance of the Various Rates - Effects of Corrections 26

2.5.1 Radiation Multipoles 26

2.5.2 Auger Rates 28

2.5 .3 Corrections - Penetration 28

$\begin{array}{llr}2.6 & \text { Further Corrections and Open Questions } & 32\end{array}$ 


\section{$-\boldsymbol{U}-$}

Chapter 3: TESTS AND COMPARISON WITH THEORY .......................... 33

3.1 Theoretical Checks $\quad 33$

3.2 Nuclear Internal Conversion Coefficients 33

3.3 Other Calculations of Penetration 35

3.4 Comparison with the Photoelectric Emission of Electrons 35

Chapter 4: applications on the experimental Results of iron And thallium. . . . . . . . . . . . . 37

4.1 General Comments on the Experiments 37

$\begin{array}{llr}4.2 & \text { Iron Results } & 37\end{array}$

4.2 .1 Features of the Data 37

4.2.2 Internal Consistency Checks 38

4.2.3 Details of the X-Ray Intensity Fits 38

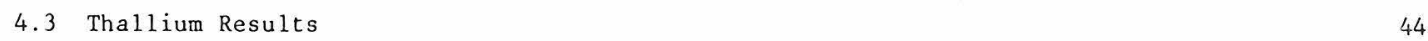

4.3.1 Features of the Data 45

4.3.2 Details of the X-Ray Intensity Fits 45

4.4 Other Experiments $\quad 45$

4.5 Conclusions on the Angular Momentum Distribution 45

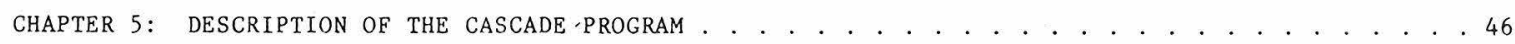

5.1 General Features $\quad 46$

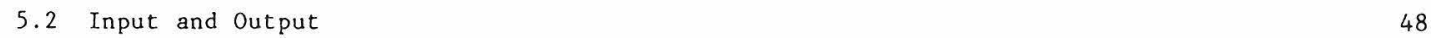

$\begin{array}{lll}5.3 & \text { Selection of Input Options } & 48\end{array}$

$\begin{array}{ll}5.4 \text { Timing } & 48\end{array}$

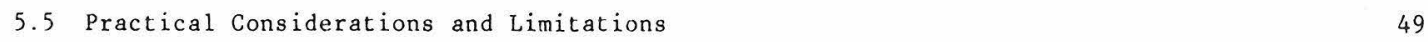

CHAPTER 6: CONCLUSIONS. . . . . . . . . . . . . . . . . . . . . . . . . . . . . 50

APpendix A: Mathematical detalls and COllection of Formulas ...................... 51

$\begin{array}{ll}\text { A.l Multipole Radiation } & 51\end{array}$

A.2 Relative Intensities of Spin Multiplets 51

A.3 Multipole Auger Transitions (no Penetration) 51

A.4 Retardation and Electronic Screening Effects in Auger Transitions 53

A.5 Penetration Effects in Auger Transitions 53

A.5.1 Muonic Integrals Done First 53

A.5.2 Electronic Integrals Done First $\quad 54$

appendix B: Sample input and output of the muonic atom cascade program. . . . . . . . . . . . . . . 64

APPENDiX C: IndeX Of NOtATION . . . . . . . . . . . . . . . . . . . . . . . . . . . . . . . 69

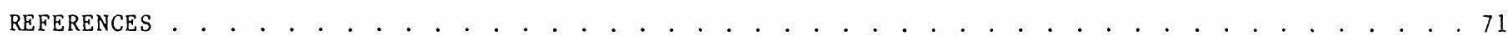


LIST OF FIGURES, TABLES, AND INPUT/OUTPUT PAGES

( $F=$ figure, $T=$ table, $P=$ input/output page)

T 0.1 Some useful properties of the muon and electron . . . . . . . . . . . . . . . . . . . 3

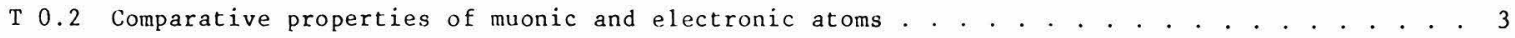

F 1.1 Capture cross sections of rubidium chloride . . . . . . . . . . . . . . . . . . . 10

F 1.2 Differential scattering cross sections of rubidium chloride................10

F 1.3 Energy distribution of uncaptured muons and capture energy spectrum . . . . . . . . . . . 12

F 1.4 Ratio of muons captured in each species of binary compounds... . . . . . . . . . . . . 12

F 1.5 Trajectory of a muon captured in an eccentric orbit . . . . . . . . . . . . . . . . . . . . 14

F 1.6 Evolution of the angular momentum distribution in the classical part of the cascade.....14

F 1.7 Angular momentum distribution at capture and later in the cascade . . . . . . . . . . . 16

F 1.8 Muon spin precession angle as a function of energy and relative angular momentum. . . . . . 16

F 1.9 Schematic representation of the setup for a semiclassically treated muon Auger transition . . 19

F 1.10 Comparison between the classical and semiclassical Auger rates. . . . . . . . . . . 19

F 2.1 Schematic representation of a radiative muon transition ..................23

F 2.2 Schematic representation of a muon Auger transition . . . . . . . . . . . . . . . . . 23

F 2.3 Effect of relativistic retardation on the Auger rates . . . . . . . . . . . . 25

F 2.4 Schematic representation of the strong dipole radiative muon transition in the cascade. . . . 25

F 2.5 Comparison of the relative intensity of the electron Auger multipole transitions. . . . . 27

F 2.6 Examples of total conversion coefficients in the cascade. . . . . . . . . . . . . . 27

F 2.7 Dependence of the electron Auger rate on energy and electronic shell. . . . . . . . . 29

F 2.8 Dependence of the conversion coeficient on energy and $y$-variable. . . . . . . . . . . 29

F 2.9 Modification of the monopole operator due to electronic screening . . . . . . . . . . 30

F 2.10 Effects of penetration on the electron Auger rates. . . . . . . . . . . . . . . . . 30

F 2.11 Derived fit for the effective nuclear charges . . . . . . . . . . . . . . . . . 31

T 2.1 Relative contributions of electronic subshells at threshold ............... 31

F 3.1 Comparison of our results with nuclear internal conversion coefficients . . . . . . . . . 34

F 3.2 Comparison of the penetration effect with other calculations. . . . . . . . . . . . . 34

F 3.3 Schematic representation of the photoelectric electron ejection . . . . . . . . . . . . 36

F 3.4 Comparison of our results with experimental photoelectric effect data . . . . . . . . 36

T 4.1 Results of the internal consistency check of the iron data. . . . . . . . . . . . . . 39

F 4.1 Fitted angular momentum distribution for iron at the beginning of the cascade...... . 39

F 4.2 Plot of the observed and fitted x-ray intensities of the muonic transitions in iron . . . . 40 
F 4.3 Some useful quantities of the cascade . . . . . . . . . . . . . . . . . . . . . . . . . .40

T 4.2 Detailed comparison of observed and fitted x-ray intensities for iron . . . . . . . . . .41

F 4.4 Fitted angular momentum distribution for thallium at the beginning of the cascade . . . . . 42

T 4.3 Detailed comparison of observed and fitted x-ray intensities for thallium . . . . . . . . . 43

F 5.1 Hierarchical arrangement of the subroutines in the program. . . . . . . . . . . . .46

T 5.1 Sample run times for best runs of the program . . . . . . . . . . . . . . . . . . . 49

F A.1 Comparison of the fitted and exact penetration functions. . . . . . . . . . . . . . 56

T A.l Multipole radiative rates . . . . . . . . . . . . . . . . . . . . . . . . . . . . 57

T A.2 Relative intensities of radiative and Auger transitions . . . . . . . . . . . . . . . . . 58

T A.3 Angular parts of multipole Auger transitions. . . . . . . . . . . . . . . . . . . . . . . 59

T A.4 Dipole Auger rates (no penetration). . . . . . . . . . . . . . . . . . . . . . . . 60

T A.5 Quadrupole Auger rates (no penetration) . . . . . . . . . . . . . . . . . . . .61

T A.6 Octupole Auger rates (no penetration) . . . . . . . . . . . . . . . . . . . . . . 62

T A.7 Selected results for radiation and electronic screening . . . . . . . . . . . . . . . . . 63

P B.1 Listing of test input . . . . . . . . . . . . . . . . . . . . . . . . 65

P B.2 Summary of input parameters . . . . . . . . . . . . . . . . . . . . . 66

P B.3 Summary of the results at the end of the cascade (last page). . . . . . . . . . . . .67

P B.4 Catalogue of x-ray intensities (last page). . . . . . . . . . . . . . . . . 68 
CHAPTER 0: INTRODUCTION

Since the discovery of new particles, scientists have been using them to probe matter in a continuous effort to unravel the structure of the world around us in its very smallest detail. In particular, negatively charged particles are mostly suited for investigating the atomic and nuclear structure, since, provided they live long enough, they can be captured by the attractive atomic potential and in their cascade to lower energies they can give information about the features of the atom and the interactions between the particle and both the atomic electrons and the nucleus.

The object of this thesis is to study the aforementioned processes with negative muons with the hope that at the end we can improve our understanding of the experimental data. More specifically we will concentrate on the quantal phase of the atomic cascade with the following goals in mind. a) Given the distribution of the muons at the onset of the cascade (sometimes referred to as "the initial l-distribution"), to calculate reliably the intensities of the muonic transition $x$ rays. This calculation depends in practice on some other physical quantities, for example, the population and rate of refilling of the electronic shells; part of the problem is the study of the dependence of the $x$-ray intensities on these not precisely known parameters. b) The inverse problem of deducing the distribution of the muons from the observed $x$-ray intensities is even more challenging. As one might expect the algorithms cannot be simply turned around! One has to work in an indirect way of searching for a good fit. The study of the feasibility of such a scheme and its reliability is equally important. c) After all work has been completed and to make the results more readily accessible to the parties interested, we provide the experimentalists with a reasonably simple tool to either predict (at least in principle) what results they should expect, or in retrospect to correlate their observations. d) As a byproduct of the above points we have derived results of theoretical interest.

We have chosen to concentrate on muons and muonic atoms for various reasons. They are much heavier than electrons (as all known negative particles are), which means that energy levels are larger in absolute value, mean radii smaller for given quantum numbers and velocities smaller for a given position in the atom, thus enabling the use of adiabatic approximation techniques. What is more important for the specific choice is that their lifetime is long enough that the whole history of such a process can be studied without decay or other terminal effect taking place in the middle of the cascade. The absence of strong interaction with the nucleus makes life a lot easier and even the weak interaction can be neglected for the purposes of the cascade since the rate of nuclear capture is far too slow compared to the cascade rates. Due to the fact that muons can be produced in copious quantities in accelerators, experimental data exist for a variety of phenomena in a multitude of elements and compounds. Weighing all these factors, one can see that muons are the ideal test particles for the study of electromagnetic phenomena in matter. To make the above points more clear and to give a short reference to the typical sizes of effects we are dealing with, we have included in tables 0.1 and 0.2 some of the relevant quantities we will be working with.

The subject of muonic atoms has been studied for some years now and a number of review articles 
have been written; among the best known are references [1-5], which include extensive original bibliographies. Rather than summarizing the results appearing in the review articles, we will only give a short account of the phenomena of interest, and proceed with the subject in question.

To present a general picture of the history of the muon, the problem can be broken down in six phases each of which has its own regions of validity and can be studied independently, given the results of the previous ones.

1) High Energy Slow-down. This process has been studied extensively by several authors [6,7,8] and it is understood quite well.

2) Low Energy Slow-down. At some energy, the velocity of the muon becomes comparable to the velocity of the outer shell electrons (of the order of $a c$ ). This condition can be written as

$$
\frac{1}{2} m_{\mu} \alpha^{2} c^{2} \geqslant E_{\mu} \approx 2 \mathrm{keV}
$$

This happens at an energy $E_{\mu} \sim 2 \mathrm{keV}$, corresponding to a wavelength $\lambda \sim 0.05 \AA$. From energies in the neighborhood of eq. (0.1) to capture, the energy loss formalism changes, one of the new features being large angle deflections. This process and the following two have been studied by other authors $[9,10,11]$, and by us $[12,13]$, although it is not yet completely understood; it will be summarized in the next chapter as a prelude to the core of the work.

3) Capture. The transition from positive energies (free state) to negative energies (bound state) is of special interest, especially if the target consists of a compound rather than a single element. At the time of capture the $Z$-distribution is fixed; the evolution of this distribution is one of our major topics of interest. In contrast to previous expectations, capture does not take place through a thermalization process of degradation of the energy by increasingly smaller steps.

4) Early Part of the Cascade. Until the energy of the muon is low enough to be adequately described by manageable quantum mechanics, the cascade must be treated in the same framework as the slow down and capture. This happens at principal quantum number $n \approx 20$ and the quantal phenomena starting to play a role are electron shell states and thresholds.

5) Late Part of the Cascade. Typically for $n \leqslant 20$ a practical quantal treatment is feasible and the results obtained can be directly compared with observations.

6) Decay or Nuclear Capture. This stage depends much on the nature of the particle and the nucleus. The low lying states of the muon can provide invaluable information about the nucleus, as among other things the nucleus has to readjust in the presence of a charged particle in its proximity. This polarization of the nuclear states due to the muon has been studied and systematized, among others, by us [14], but these results are beyond the scope of this thesis.

The bulk of this work is the exploration of step 5, given the outcome of steps 1 - 4 (or conversely, we might be able to say something about the preceding steps $1-4$ ). One might ask why do We need a new cascade program. Although the need will become apparent as we proceed, it is simple to 
TABLE 0.1

Some Useful Properties of the Muon and Electron ${ }^{\text {a) }}$.

\begin{tabular}{|c|c|c|}
\hline & MUON & ELECTRON \\
\hline Mass & $\begin{array}{ll}105.65948(35) & \mathrm{MeV} \\
206.7686(7) & m_{e}\end{array}$ & $0.5110034(14) \mathrm{MeV}$ \\
\hline Free lifetime (mean 1ife) & $2.197134(77) \mu \mathrm{sec}$ & stable \\
\hline $\begin{array}{l}\text { Nuclear capture lifetime } \\
\text { in atom }(Z, A) \text { (mean life) }\end{array}$ & $\sim 0.006 \frac{1}{z_{\text {eff }}^{2}} \frac{1}{\left(1-3 \frac{A-Z}{2 A}\right)} \sec ^{b)}$ & stable \\
\hline Magnetic moment & $\left.1.001165897(27) \frac{e \hbar}{2 m_{\mu} c} \quad c\right)$ & $1.0011596567(35) \frac{e \hbar}{2 m_{e^{c}}}$ \\
\hline Compton wavelength & $1.867590(7) \mathrm{fm}$ & $386.15905(64) \mathrm{fm}$ \\
\hline
\end{tabular}

a) Entries from ref. [16].

b) Semiempirical formula from ref. [17].

c) Numerical value corrected for typographical error as per a later edition of ref. [16].

TABLE 0.2

Comparative Properties of Muonic and Electronic Atoms a).

\begin{tabular}{|c|c|c|c|c|}
\hline QUANTITY & Definition & Expression & $\begin{array}{l}\text { Ratio between muonic } \\
\text { and electronic atoms }\end{array}$ & $\begin{array}{l}\text { Typical values for } \\
\text { muonic atoms }\end{array}$ \\
\hline Atomic radius & $\left\langle\frac{1}{r_{n}}\right\rangle^{-1}$ & $\frac{n^{2} \hbar^{2}}{2 e^{2}} \frac{1}{m}$ & $\frac{m_{e}}{m_{\mu}} \approx \frac{1}{207}$ & $5 \mathrm{fm} \quad(Z \approx 50)$ \\
\hline Energy levels & $E_{n}$ & $-\frac{(2 \alpha)^{2} c^{2}}{2 n^{2}} m$ & $\frac{m_{\mu}}{m_{e}} \approx 207$ & few MeV (med. $Z, n=1$ ) \\
\hline Fine structure & $\left\langle\frac{(\boldsymbol{L} \cdot \boldsymbol{\sigma}) 2}{m^{2} r^{3}}\right\rangle$ & $\propto Z^{4} m$ & $\frac{m_{\mu}}{m_{e}} \approx 207$ & $\begin{aligned} .2-.6 \mathrm{MeV} & \text { in heavy } \\
& \text { atoms }\end{aligned}$ \\
\hline $\begin{array}{l}\text { Velocity at a } b \text { ) } \\
\text { given point }\end{array}$ & $v$ & $\propto \sqrt{\frac{1}{m}}$ & $\sqrt{\frac{m}{m_{\mu}}} \approx \frac{1}{14}$ & $\begin{aligned} 5 \times 10^{-4} \mathrm{Zc} & \text { for electron } \\
& \mathrm{K}-\text { shell }\end{aligned}$ \\
\hline
\end{tabular}

a) Some entries from corresponding table in ref. [2].

b) Assuming that the total energy is given. 
note here that the predecessor of our code [15], the so called "Hüfner program", was written in order to describe results for muons in very low states (typically $n \leqslant 7$ ). That program included dipole radiative transitions and the dipole part of the muon-electron interaction. The monopole Auger rates were treated in a very crude way. It was assumed that the muon orbit is much smaller than the electron orbits and thus the penetration could be neglected. For its intended range it performs well. However, in the later years experimental results have become available for transitions from much higher levels. Extension of this program to high quantum numbers is insufficient if not disastrous! A host of otherwise negligible effects has been ignored, which at higher quantum numbers plays a key role. Other multipoles besides dipole, and the so called penetration correction to the Auger rates are two outstanding ones. Along with some procedural improvements, our program takes into account all important effects.

To preview the upcoming chapters, first we give some theory about the early stages of the muon, so that we can justify our later actions. Next comes the development of the theory with all ramifications of our expansion considered. Chapter 3 has the comparison with other theoretical and experimental results, that gives us confidence about our treatment. Chapter 4 discusses the application mostly intended for the program, namely the comparison and fitting of the experimental data for the iron and thallium $x$ rays. Chapter 5 is dedicated to the computer program and its details. The appendices include a compilation of the formulas involved, a reproduction of some typical input and output and an index of the notation used.
\end{abstract}


CHAPTER 1: INITIAL HISTORY OF THE MUON IN THE ATOM

To understand the main part of this work and to appreciate its contribution to the whole framework, we must examine the history of the muon prior to its quantal cascade. Moreover, we will gain insight about some parameters needed at the onset of the quantal calculation. Since this work has been presented elsewhere in detail [9-13], we will limit ourselves to an overview of the subjects with particular emphasis on the concepts most relevant to our main topic. References [12,13] constitute our contribution to the classical part of the muon history. The work has been a collaboration of several researchers ( $c f$. author list of papers) and the personal involvement of the author has been in the systematization of the details and development of the computer programs, rather than in the planning of the major steps.

1.1. Energy Loss from the Continuum. As the muons are slowed down in a material medium (target), starting from high energies, their velocities become comparable (or smaller) to the typical Fermi velocities of the atomic electrons. Whereas the mechanisms of energy loss of swift charged particles are well known and understood $[6,7,8]$, there are several problems associated with the corresponding processes when the muons are slower than the electrons in the atom. The stopping power of a target. as described, for example, by the expression [1]

$$
-\frac{\mathrm{d} E}{\mathrm{~d} s}=\frac{4 \pi e^{4}}{m_{\mu} v_{\mu}^{2}} N Z\left\{\ln \frac{2 m_{\mu} v_{u}^{2}}{I}+\ln \frac{1}{1-\beta^{2}}-\beta^{2}-\frac{C}{2}-\frac{1}{2} \delta\right\},
$$

where $d E / d s$ is the energy loss per unit path length, $\beta=v_{\mu} / c, N$ is the density of atoms per unit volume, $I$ is the mean excitation energy, and $C$ and $\delta$ are correction parameters, works fine at high energies, but such a description breaks down at low speeds, one reason being that the mean excitation energy $I$ is hard to estimate. Thus quantum mechanical methods become complicated (the Born approximation fails, since the action integral is larger than $\hbar$ ) and unreliable, partly due to the large number of bodies involved. As early as thirty years ago, Fermi and Teller in a comprehensive paper [18] laid the fundamental physical picture, using classical methods with rough predictions of physically observable quantities (capture rates etc.). Since that time a compilation of several experiments by a variety of groups [19-23] demonstrate that the early predictions, which depend smoothly on the atomic number $Z$, in reality show variations, which can be correlated with the atomic shell structure or the chemical details of the environment. Being motivated by the basically sound treatment of the classical description of the phenomena initiated by Leon and Seki [9], we undertook the task $[12,13]$ of refining the theory by including as many features as deemed feasible. The result is an improved description of the processes of slowing down, capture and classical cascade.

1.1.1. Justification of the Classical Approach. The fact that until very low energies the muon wave function has many oscillations over the extent of the atom (e.g., at a muon energy of a few eV and most muons get captured at higher energies - their wavelength is much smaller than $1 \AA$ ) suggests the use of a classical description of the muon motion, unlike the perturbation style approach of the 
higher energies. At energies lower than about $2 \mathrm{keV}$ the muon-electron interaction is so strong that first order quantal calculations are simply wrong! Nevertheless, what makes a calculation still manageable in this region is the fact that the muon (at position $\boldsymbol{x}^{\circ}$ ) moves slowly compared to the nearby electrons. The ratio of velocities is given by

$$
\frac{v_{\mu}}{v_{e}}=\sqrt{\frac{m_{e}}{m_{\mu}} \cdot \frac{E_{\mu}-V(r)}{E_{e}-V(r)}},
$$

where we have assumed that the potential energy $V(\boldsymbol{r})$ is the same for both particles. For the low muon entrgies and electrons close to the Fermi surface we find that over most of the atom the muon moves with a velocity much smaller than the velocities of the electrons, that is

$$
\frac{v_{\mu}}{v_{e}} \approx \sqrt{\frac{m_{e}}{m_{\mu}}} \approx \frac{1}{14} .
$$

This means that the motion of the slow muonic perturbation causes the electronic wave functions to adjust rapidly to the almost static field of the muon at $\boldsymbol{r}(t)$, and so we are dealing with a continuously changing two center problem for the atomic electrons. As a result of the presence of a negatively charged "impurity" in the atom, the density of electrons is diminished around the muon and the muon-electron interaction is shielded at large distances, the shielding length being of the order of $[18]$

$$
1 / \kappa=\sqrt{\alpha_{0} \lambda_{e}},
$$

where the local reduced electron wavelength $\lambda_{e}$ is given by

$$
\lambda_{e}=\hbar / m_{e} v_{F} \quad,
$$

and it depends on the Fermi velocity $v_{F}$ at that point. This reduction of the interaction corroborates the reduction of the lowest order perturbation term (one quantum exchange between the muon and the atom). From the slow motion of the muon, it follows that the maximum energy loss in each quantal collision is limited by the classical maximum momentum transfer given by [18]

$$
\Delta E_{\max }=2 m_{e} v_{F_{\mu}}(t) \quad,
$$

which is $\approx 2 / 7$ times the kinetic energy of the muon. Actually the most probable energy loss is only about $1 / 10$ of the maximum value given in eq. 1.6. This means that the trajectory of the muon is not greatly modified by the energy loss. These arguments fully justify the use of the classical description of the muon trajectory and its interaction with the electrons.

1.1.2. Description of the Energy Loss Mechanisms. The starting point is the probability density distribution of the energy transfer $\varepsilon=\hbar \omega$ from the muon to the electron cloud during the time interval $\mathrm{d} t$, given by [26] 


$$
\frac{\mathrm{d}^{2} p(\omega)}{\mathrm{d} \omega \mathrm{d} t}=\frac{e^{2}}{\pi \hbar v_{\mu}(t)} \int_{\omega / v}^{\infty} \frac{\mathrm{d} k}{k} \operatorname{Im}\left(\frac{1}{\varepsilon^{2}(k, \omega)}\right),
$$

where $\varepsilon^{2}(k, \omega)$ is the Fourier transform of the (local) longitudinal dielectric constant of the electrons treated as a free Fermi gas. The theory of electronic stopping, which we are employing here was developed by Lindhard and collaborators [24-26]. In that approach the electrons are treated as a Fermi gas and the dielectric constant is calculated in the time dependent self-consistent Hartree-Fock approximation. We have studied the ramifications of the theory including the variations of the electron density over the atom [private work - unpublished] and we have found that the corrections are too small to significantly change the basic results. From eq. (1.7) one can calculate the stopping power, by forming the average energy loss (per unit path length), i.e.,

$$
\frac{\mathrm{d} E}{\mathrm{~d} s}=\frac{1}{v_{\mu}} \frac{\mathrm{d} E}{\mathrm{~d} t}=\frac{1}{v_{\mu}} \int \hbar \omega \frac{\mathrm{d}^{2} p(\omega)}{\mathrm{d} \omega \mathrm{d} t} \mathrm{~d} \omega
$$

and performing the indicated integrations in (1.7) and (1.8) using the relevant approximations for the dielectric constant described in ref. [26]. In the present treatment [13] we keep the differential form of eq. 1.7 with respect to the energy loss and we simply integrate (1.7) along the muon trajectory through the atom to obtain the spectrum of energy losses $d p(\omega) / d \omega$ along such a trajectory, specified by the energy of the muon (at infinity) and the impact parameter. This resulting distribution is sharply peaked at losses of a few eV and it falls off at high energies (up to the maximum energy loss allowed of $\left.2 m_{e} v_{\mu}(t) v_{F}\right|_{\max }$, eq. 1.6) only as $1 / \omega^{2}$; large energy losses are rare, but contribute significantly to $\mathrm{d} E^{\prime} / \mathrm{d} s$ at small distances. The calculation of $\mathrm{d} p(\omega) / \mathrm{d} \omega$ for the collection of impact parameters from zero to the size of the atom and for energies from several hev to zero, is the result of this integration and all relevant quantities can be obtained from such a collection of distributions.

One additional mathematical complication, which was in fact expected, is that the total probability $p$ for any energy loss, given by

$$
p=\int \frac{\mathrm{d} p(\omega)}{\mathrm{d} \omega} \mathrm{d} \omega
$$

is in practice larger than unity. This simply means that the possibility of multiple scatterings in one atomic encounter is nonnegligible. In view of this we must consider the probability $\mathrm{d} P_{t}(\varepsilon) / d \varepsilon$ that at time $t$ the muon has lost the energy $\varepsilon$ (corrected for multiple scatterings). Such a quantity obeys the integral equation [13]

$$
\frac{d}{d t}\left(\frac{d P_{t}(\varepsilon)}{d \varepsilon}\right)=\int\left\{\frac{d P_{t}(\varepsilon-\delta)}{d \varepsilon}-\frac{d P_{t}(\varepsilon)}{d \varepsilon}\right\} \frac{d^{2} P(\delta)}{d \delta d t} d \delta
$$

which connects the rate of change of $\mathrm{dP}_{t}(\varepsilon) / \mathrm{d} \varepsilon$ (LHS) to the accumulation (1st term RHS) and depletion (2nd term RHS) of $\mathrm{dP}_{t}(\varepsilon) / \mathrm{d} \varepsilon$ via the "one interaction" energy loss distribution 


\section{$-8-$}

$d^{2} p(\omega) / d \omega d t$. Equation 1.10 can be solved analytically in some approximation (ref. [13] treats a realistic case) or numerically by an iterative scheme.

In our early treatment of the problem [12] we used the average energy loss per unit path length, i.e., stopping power (1.8), as the basic quantity. When the corresponding integral is evaluated, one finds that the stopping power is proportional to the velocity of the muon and, therefore represents a frictional force. Specifically we get

$$
\begin{aligned}
\frac{d E}{d s} & =-\frac{4}{3 \pi} \frac{m_{e}^{2} e^{4}}{\hbar^{3}} \frac{1}{2\left(1-\frac{1}{3} x^{2}\right)^{2}}\left\{\ln \left(\frac{1-\frac{2}{3} x^{2}}{x^{2}}\right)-\frac{1-\frac{1}{3} x^{2}}{1+\frac{2}{3} x^{2}}\right\} v_{\mu}, \\
X(r) & =\left\{\frac{e^{2}}{\pi \hbar v_{F}(r)}\right\}^{\frac{1}{2}} .
\end{aligned}
$$

Such a force correctly describes the average behavior of the muon, but it is inadequate for the description of the capture process [13]. This is because the question whether the muon is captured or not is decided by the condition that the energy loss is larger than the total energy minus the centrifugal potential barrier in the radial motion and not by the condition that the average energy loss is large enough.

1.1.3. Comments on the Calculation. The detailed description of the atomic cloud presents some problems in a classical approach. We have used both the Lenz-Jensen potential [27], an analytic approximation of the Thomas-Fermi model adjusted for densities near the origin and at large distances, and the Hartree-Fock potential [28]. The final results for all three potentials are similar. Note that in all cases the probability is calculated using the assumptions of the Fermi gas model (for the derivation of the dielectric constant), and only the details of the charge density are dependent on the particular potential used - this hybrid setup does not satisfy the Thomas-Fermi equations for the charge density. This regime breaks down at small radii $\left(r<a_{0} / Z\right)$ and large ones $\left(r>\alpha_{0}\right)$. For small distances the statistical density is wrong (the Hartree-Fock is more accurate), but the muon spends little time there; on the other hand, the statistical model is not applicable for very low electron densities - but at large distances the probability for any significant energy losses is trivially small. Another situation where this approach fails is at very low bombarding energies $\left(E_{\mu} \leqslant E_{\text {chemical }}\right)$, where chemical effects may become important.

In addition to the energy loss from collisions with electrons, the muon experiences energy loss by electromagnetic radiation. The radiative losses have been treated in the lowest order (dipole) [29] and they give appreciable damping only for orbits coming very close to the nucleus ( $x \leqslant 10^{-3} \AA$ ), arising from the high accelerations at the turning pericenter points, since the dipole radiation power is proportional to the square of the acceleration.

1.1.4. Equations of Motion and Their Solution. In the presently adopted probability oriented formulation it is relatively hard to incorporate the changes in the muon orbit due to the energy loss distribution; this was done in the case of the frictional force. A Monte Carlo type of calculation is 
still possible, but quite time consuming. As pointed out above, the muon during traversal of the atom on the average exchanges one quantum with the electrons and changes its total energy in a not very significant way. Thus, we decided to describe the trajectory by the elastic energy conserving one. Along this trajectory, the differential energy loss probability (1.7) is integrated; as we have seen, the errors introduced by moving along the elastic trajectory should not be too large, perhaps except at very low energies, where our free atom theory breaks down anyway. The collection of energy loss probability distributions as a function of the original muon energy and impact parameter constructed in sect. 1.1 .3 will be used in the following in order to obtain more useful quantities.

1.1.5. Cross Sections. The quantities of interest are the (differential) cross section for the muon of energy $E$ to lose energy $\varepsilon$ and the (total) cross section for the muon of energy $E$ to be captured. The first is obtained by integrating $\mathrm{dP}_{t}(\varepsilon) / \mathrm{d} \varepsilon$ over the atomic cross section according to

$$
\frac{\mathrm{d} \sigma_{\varepsilon}(E)}{\mathrm{d} \varepsilon}=2 \pi \int_{0}^{d} b \mathrm{~d} b \frac{\mathrm{dP}\left(\varepsilon<E-E_{\mathrm{bar}}(b)\right)}{\mathrm{d} \varepsilon},
$$

where $d P(\varepsilon) / d \varepsilon$ has been integrated along the muon trajectory (sect. 1.1.4), $E_{\text {bar }}$ is the height of the effective potential barrier [ef. ref. [12], fig. 4] and $d$ is a parameter characterizing the size of the atom [ef. ref. [12], eq. 5.1]. Quantal corrections for small impact parameters, large energy losses and bremstrahlung have been included in the actual calculation [13].

The capture cross section is a special case of (1.11) in the region $\varepsilon \geq E-E_{\text {bar }}$, given by

$$
\sigma_{\text {cap }}(E)=2 \pi \int_{0}^{d} b \mathrm{~d} b \int_{E-E_{\mathrm{bar}}(b)}^{\infty} \frac{\mathrm{dP}(\varepsilon)}{\mathrm{d} \varepsilon} \mathrm{d} \varepsilon .
$$

1.2. Muon Capture from the Continuum. Equation 1.12 describes the cross section for capture; figure 1.1 shows a typical situation for the case of rubidium chloride. Note the exponential drop at high energies, which nonetheless cannot be ignored. One would think that at the low energies (below $\sim 10 \mathrm{eV}$ ) capture would be the strongest and above that almost none should occur; this is certainly not the case. The reason is that there is a dynamic competition between scattering and capture. To further elucidate the issue, figure 1.2 shows the associated scattering cross section (several energies) of fig. 1.1. The importance of the high energy tails in these curves should be noted.

We now define the function $P(E)$ as the probability per unit energy and per unit area of having a muon of energy $E$ (not captured) in the medium. The evolution of $P(E)$ as a function of $E$ is governed by the integral-differential equation of the steady state condition [12]

$$
P(E)\left\{\sigma_{\text {cap }}(E)+\int_{0}^{E} \frac{\mathrm{d} \sigma_{\varepsilon}(E)}{\mathrm{d} \varepsilon} \mathrm{d} \varepsilon\right\}=\int_{0}^{\infty} \frac{\mathrm{d} \sigma_{\varepsilon}(E+\varepsilon)}{\mathrm{d} \varepsilon} P(E+\varepsilon) \mathrm{d} \varepsilon,
$$

where the LHS is the depletion of muons by capture or scattering to lower energies and the RHS is the feeding from the higher energies. The possibly divergent cross sections at $\varepsilon=0$ exactly cancel, thus 
Figure 1.1

Capture cross section of rubidium chloride.

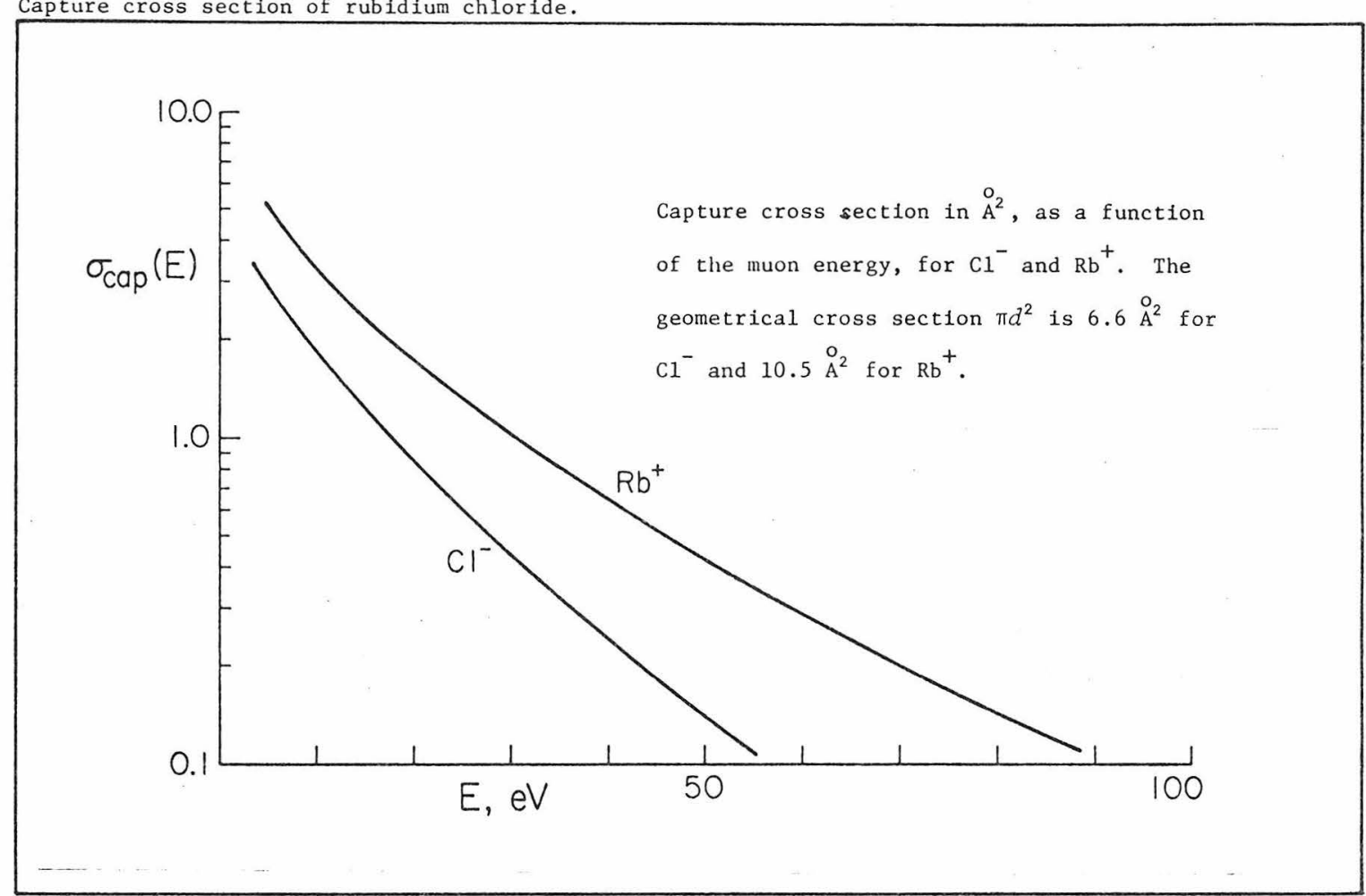

Figure 1.2

Differential scattering cross section of rubidium chloride

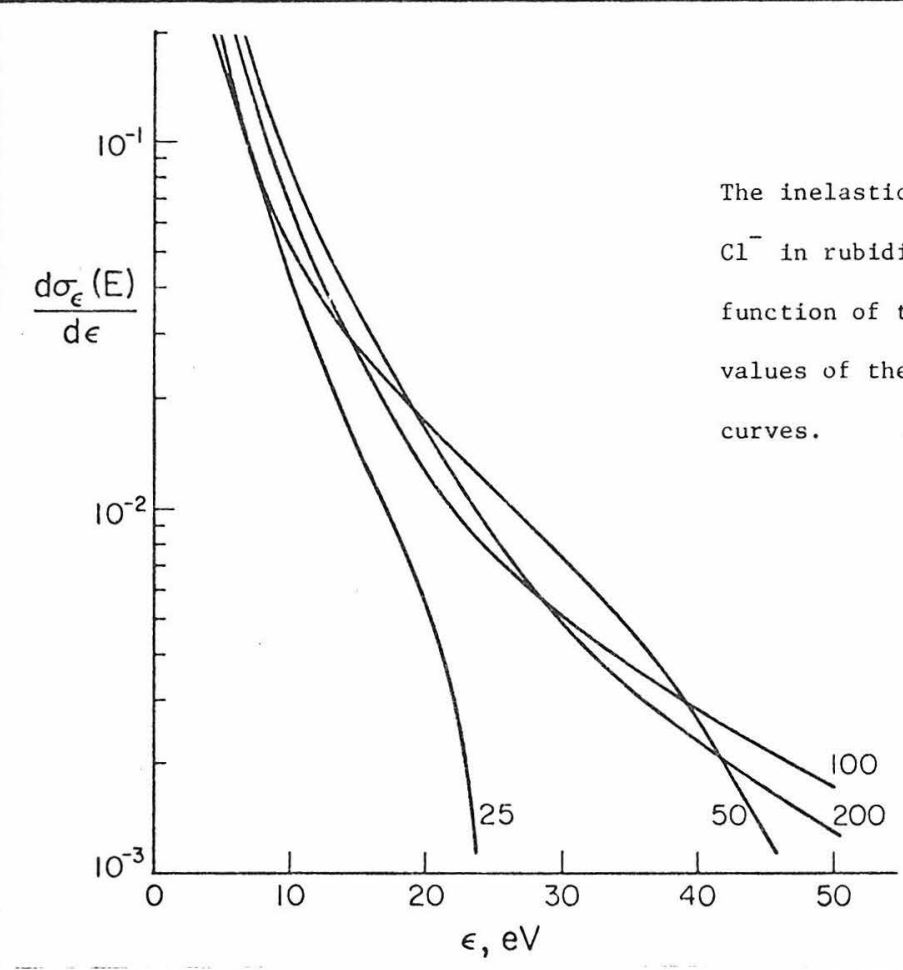


the lower limits of integration on both sides of eq. 1.13 could be replaced by some small energy $\varepsilon_{0} 0$. Figure 1.3 shows a numerical solution of this equation for the case of $\mathrm{Rb}^{+} \mathrm{i}$ used in figs. 1.1 and 1.2. The slight increase of the function $P(E)$ above unity at muon energies between 100 and $200 \mathrm{eV}$ is related to the detailed form of the inelastic cross section. Capture starts at around $50 \mathrm{eV}$, where the capture cross sections are still small. Only about $25 \%$ of the muons are captured near zero energy in this fairly typical case. This establishes roughly the relative role of capture in which the "free atom" picture described above is applicable. At smaller energies the chemical structure of the target plays a decisive role.

1.2.1. Angular Momemtum at Capture. The angular momentum distribution of the muons is of importance at the onset of the quantal phase of the cascade, and it either has to be obtained from the earlier stages of the calculation, or otherwise fitted from the data. A combination of the two is even better, since so many unaccounted phenomena contribute to its precise definition. This distribution at the moment of capture can be readily calculated from the capture probabilities and the distribution

$P(E)$. Indeed, if we define the number of muons per unit energy captured with angular momentum $L$ (in units of $\hbar$ ) as $N(L)$, then the differential form of this function due to muons captured within $\Delta L$ of $L$ is given by

$$
\Delta N(L)=\frac{\hbar^{2} L \Delta L}{m_{\mu}} \int_{0}^{\infty} \frac{P(E)}{E} \mathrm{~d} E 2 \pi \int_{0}^{d} b \mathrm{~d} b \Pi\left(b^{2}-b_{0}^{2}(L, \Delta L)\right) \int_{E^{\prime}-E_{\mathrm{bar}}(b)}^{\infty} \frac{\mathrm{dP}(\varepsilon)}{\mathrm{d} \varepsilon} \mathrm{d} \varepsilon,
$$

where the function $\Pi\left(b^{2}\right)$ assures that the angular momentum is between $L$ and $L+\Delta L$, being defined by

$$
\pi\left(b^{2}-b_{0}^{2}(L, \Delta L)\right)=\left\{\begin{array}{ll}
1 & \text { if } L(b) \in[L, L,+\Delta L] \\
0 & \text { otherwise }
\end{array} .\right.
$$

Also $b_{0}(L, \Delta L)$ stands for the solution of the impact parameter as a function of the angular momentum (from $\left.\hbar L=\left(2 m_{\mu} E\right)^{\frac{1}{2}} b\right)$. In the limit of infinitesimal $\Delta L$ the integration over $L$ collapses and a simpler result is obtained, viz.

$$
\Delta N(L)=\frac{h^{2} L \Delta L}{m_{\mu}} \pi \int_{0}^{\infty} \frac{P(E)}{E} \mathrm{~d} E \int_{E^{\prime}-E_{\mathrm{bar}}(L)}^{\infty} \frac{\mathrm{d} P(\varepsilon)}{\mathrm{d} \varepsilon} \mathrm{d} \varepsilon
$$

If the integrals depending on $L$ (or equivalently on $b$ ) do not in fact depend on $L$, then we get that

$$
\Delta N(L) \propto L \Delta L \quad,
$$

which is the well known statistical distribution. At the present time we have not studied in detail the behavior of these integrals and the deviation of the angular distribution from the statistical one. This distribution as evolved in the classical part of the cascade, can serve as a guideline for the initial 2 -distribution of the quantal cascade.

1.2.2 Compounds. For completeness we should mention that the above techniques can be applied to 
Figure 1.3

Energy distribution of uncaptured muons and capture energy spectrum.

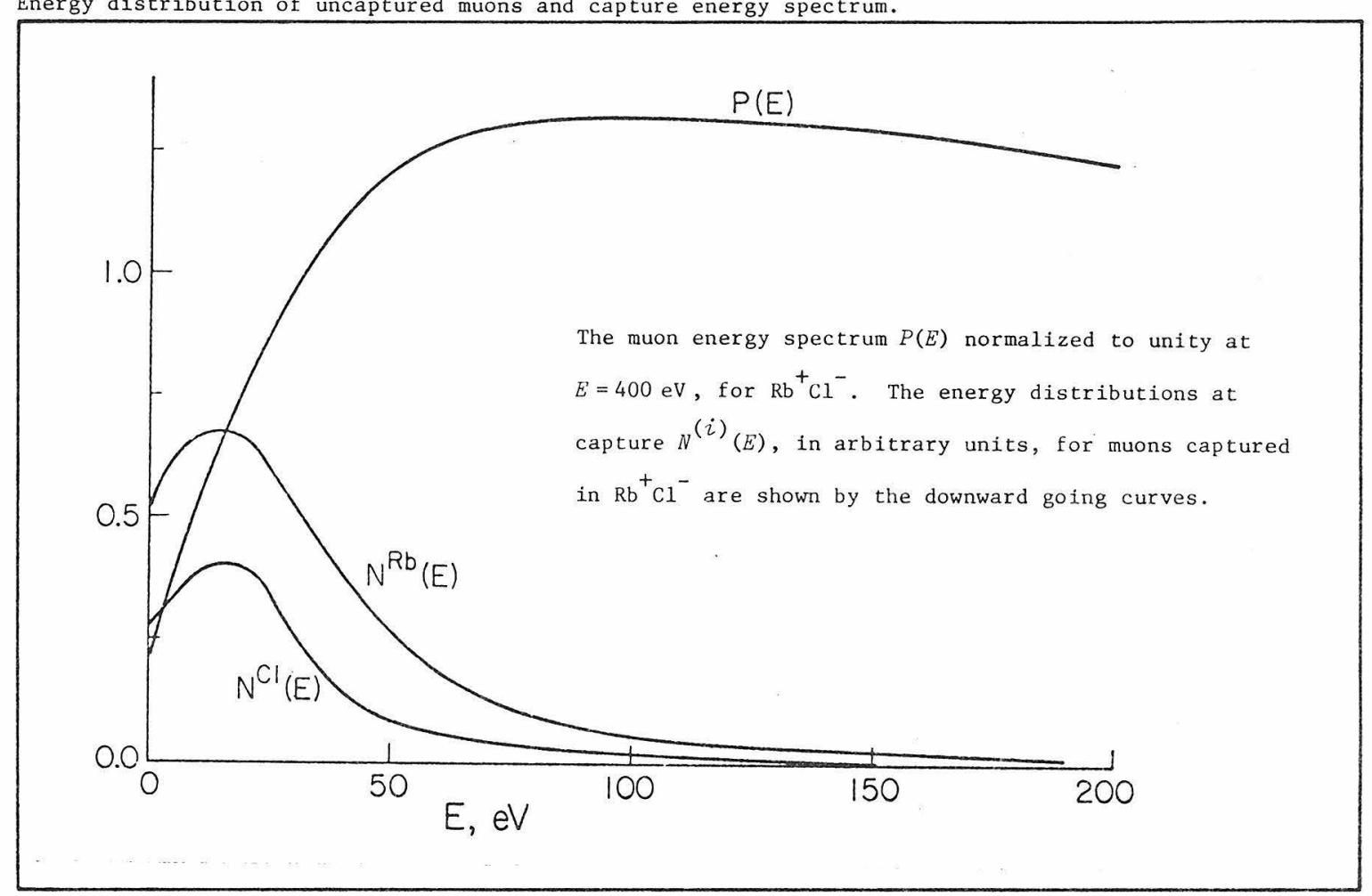

Figure 1.4

Ratio of muons captured in each species of binary compounds.

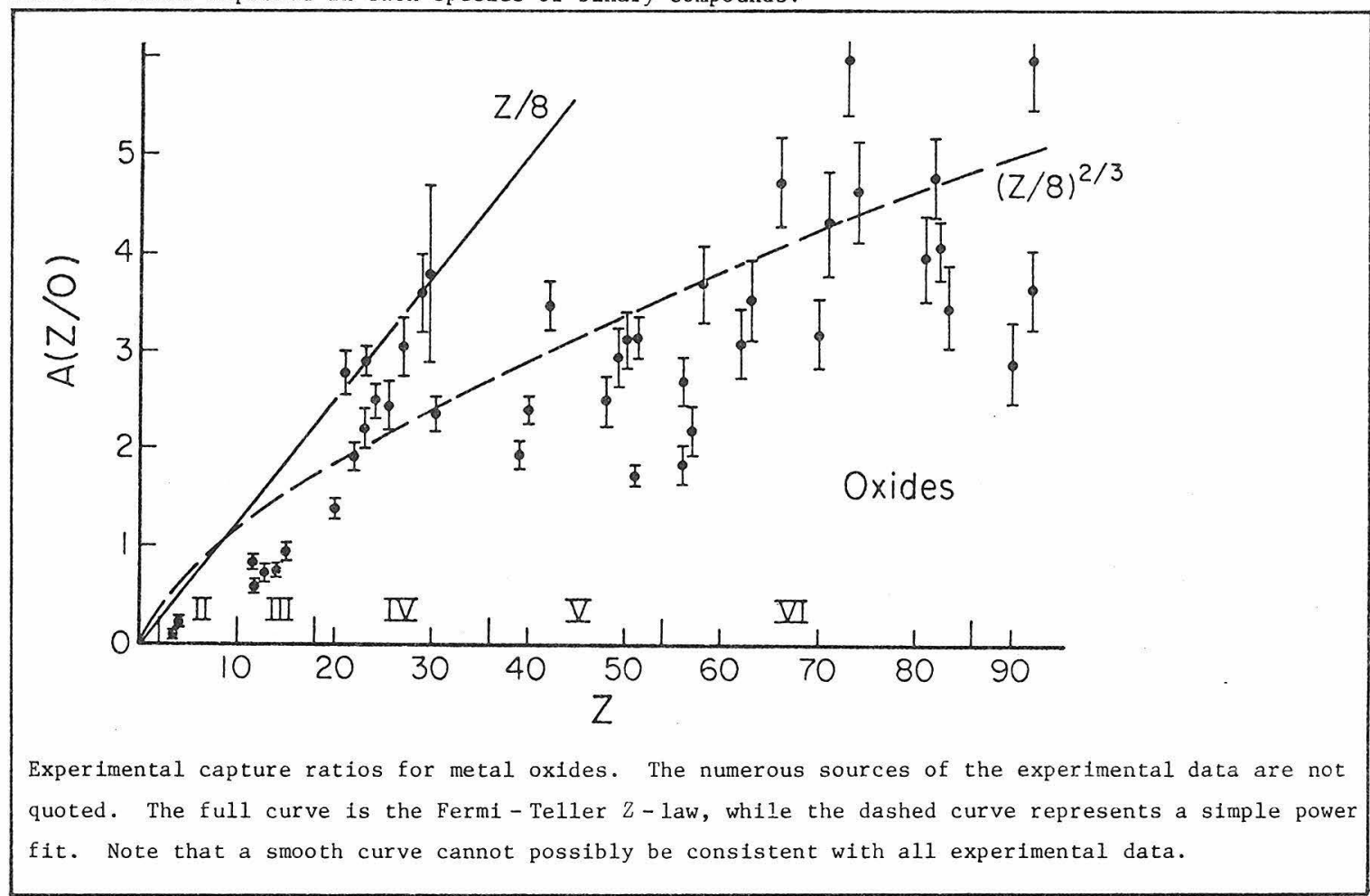


molecules, where the various constituents are treated as homogeneous mixtures, disregarding chemical bonds (but taking into account transfer of charge and distortion of the atomic potential). One of the advantages of treating binary compounds is the extensive collection of data (as an example a large number of oxides has been tested). In our treatment, each species of atoms or ions enters with its own capture and scattering cross section in the generalized version of eq. 1.13 for the unique function $P(E)$. A separate 2 -distribution can be evaluated for each species, and moreover, the ratio of muons captured in the various atoms can be calculated by noting that the number of muons per unit energy captured at energy $E$ for species $i$ is

$$
N^{(i)}(E)=n_{i} P(E) \sigma_{\text {cap }}^{(i)}(E),
$$

where $n_{i}$ is the (fractional) concentration of $i$. The total number captured in $i$ is obtained by integrating (1.18)

$$
N_{i .}=\int_{0}^{\infty} N^{(i)}(E) \mathrm{d} E
$$

For a two component compound we can form $N_{1} / N_{2}$, which can be derived from the experimental ratios of $x$-ray intensities. It has been predicted [18] under simple assumptions that this ratio is equal to the ratio of atomic numbers $Z_{1} / Z_{2}$; although there is a wild variation in the data, it seems that the observed ratios are closer to unity than the "z-1aw". As we have pointed out in ref. [13] a considerable uncertainty in these numbers can be attributed to the chemical effects of the compound in the energy region of a few ev. Our calculations, although not consistently in agreement with the data, seem to be closer to experiment. To illustrate how complicated the matter is, figure 1.4 shows some data with attempted fits to describe them.

1.3. Classical Part of the Cascade. The reasons of sect. 1.1 for the use of classical methods apply here too, since energies are small and the quantum numbers of the muon are large. We are going to describe the energy and angular momentum losses by the same equations of motion, but in doing so we must update the orbit of the muon (this is done in the average formalism of ref. [12]), so that it will actually enter more tightly bound orbits. Direct computation is lengthy (the muon typically makes thousands of loops before descending to the region where the quantal cascade takes over). This calculation has been performed for a selected number of cases mainly as a check on the more indirect methods below. To give a visual idea of what a high eccentric orbit looks like, we have included figure 1.5. The alternative to following the orbit is to derive an equation describing the evolution of $L$ and $E$. It is convenient to express $L$ as a fraction of the maximum angular momentum $L_{\text {max }}(E)$ (corresponding to the circular orbit at energy $E$ ) This maximum $L$ is determined from the equation

$$
E=\frac{L_{\max }^{2}(E)}{2 m_{\mu} r^{2}}+V(r),
$$

and the condition for maximum 
Figure 1.5

Trajectory of a muon captured in an eccentric orbit.

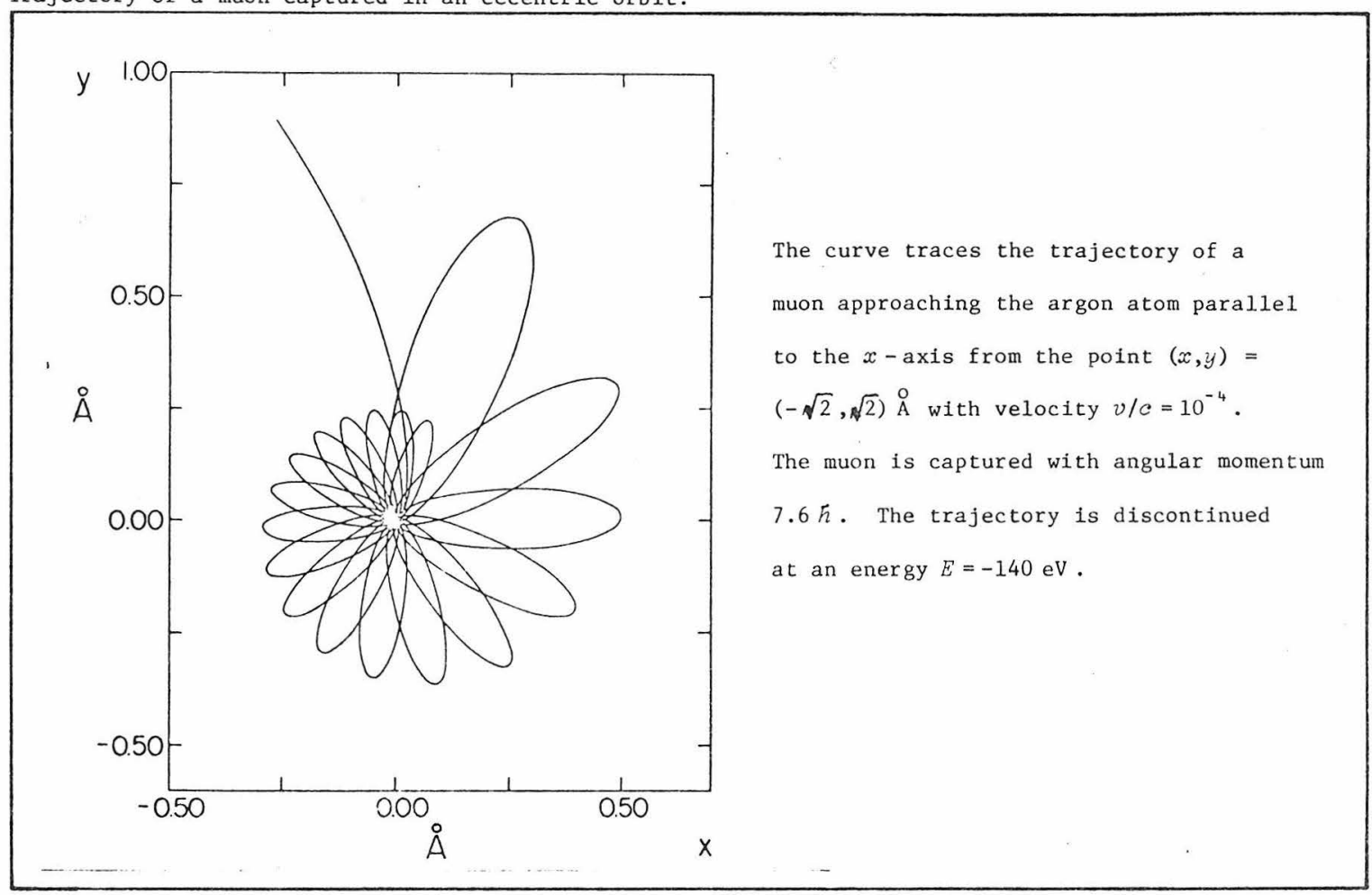

Figure 1.6

Evolution of the angular momentum distribution in the classical part of the cascade.

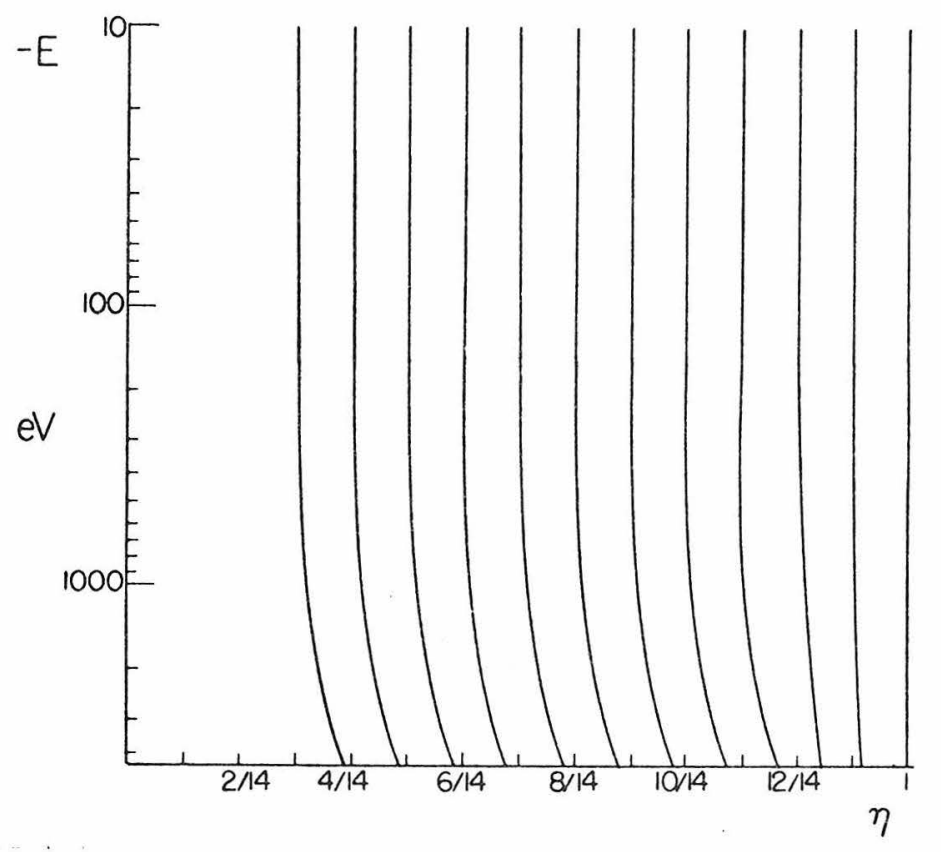

The variation of the parameter $n$ (cf. eq. 1.22) with energy $E$ in argon is shown for energies between zero and the electron $\mathrm{K}$ - shell energy for several initial choices of $\eta$. The parameter $\eta$ is nearly constant and the shape of the angular momentum distribution from zero energy of the muon to the $\mathrm{K}$ - shell electron binding energy is almost unchanged. 


$$
\frac{\mathrm{d}}{\mathrm{d} r}\left(\frac{L_{\max }^{2}(E)}{2 m_{\mu} r^{2}}\right)+V(r) \quad=0 .
$$

If $L$ is expressed as a fraction of $L_{\max }\left(E^{\prime}\right)$, i.e.,

$$
\eta(E)=L(E) / L_{\max }(E)
$$

we find that the change of $n$ over an entire loop of the muon is related to the energy loss $\triangle E$ over the same period $t_{1} \Rightarrow t_{2}$ by

$$
\frac{1}{\eta} \frac{\Delta \eta}{\Delta E}=\frac{\int_{t_{1}}^{t_{2}}\left[f(r)+\frac{\gamma}{m_{\mu} r} \frac{\mathrm{d} V}{\mathrm{~d} r}\right] \mathrm{d} t}{\int_{t_{1}}^{t_{2}}\left[2 f(r) T+\frac{\gamma}{m_{\mu}}\left(\frac{\mathrm{d} V}{\mathrm{~d} r}\right)^{2}\right] \mathrm{d} t}-\frac{1}{L_{\max }} \frac{\mathrm{d} L_{\max }}{\mathrm{d} E},
$$

where $f$ is the friction coefficient ( $c f$. eq. 2.10, ref. [12]) and $\gamma=2 / 3 e^{2} / c^{3}$ coming from the radiation. The use of the friction (i.e., average energy loss) is a demerit of the treatment at this point, but it can be justified by noting the relatively long time spent by the muon on this trajectory. Equation 1.23 can be solved numerically and a sample case is shown in figure 1.6. The vertical almost straight lines show that although there is an overall loss of angular momentum (a smaller orbit cannot support as much $L$ as a large one), the quantity $\eta$ is preserved and therefore the shape of the $l$-distribution remains unchanged. For the circular orbits $(n=1)$ this is exactly correct, whereas extremely eccentric orbits converge to more circular ones. In practice the distribution tends to accumulate some high $L$ states, so that at the point where the quantal cascade takes over, the distribution is visibly steeper than the one at capture, as exhibited by figure 1.7.

1.4. Depolarization of the Muons. [30] The study of the loss of polarization of the captured and cascading muons can reveal information about the (weak) muon-nucleus interaction in addition to the solid state and chemical applications. The muon beams produced by decaying pions are almost fully polarized and the residual polarization in the muonic atom is measured from the asymmetry of the decay electrons, or from the circular polarization of the muonic $x$ rays [31]. Our goal is to study the deflection of the spin of the muon by electron collisions and by the spin-orbit interactions. Previous discrepancies by factors of 1.5 to 2 between the theory [5] and experiment, accounted by a slower cascade or additional depolarization mechanisms, have been substantially bridged and such additional hypotheses may be unnecessary in many cases. The two mechanisms of depolarization are outlined next, the quantal case following.

1.4.1. Spin Deflection in Muon-Electron Collisions. The Larmor precession angular velocity in such a collision (where the slow muon is treated as stationary) is given by

$$
\Omega \hbar=\frac{\mu_{e} \cdot \mu_{\mu}}{r^{3}}-3 \frac{\left(\mu_{e} \cdot \mathbf{r}\right)\left(\mu_{\mu} \cdot \boldsymbol{r}\right)}{r^{5}},
$$

where $\boldsymbol{\mu}$ is the magnetic moment and $\boldsymbol{r}$ is the radius vector. Integrating $\Omega$ over a muon loop (in a simplified fashion of a straight trajectory through the center of the atom) and inserting an estimate 
Figure 1.7

Angular momentum distribution at capture and later in the cascade.

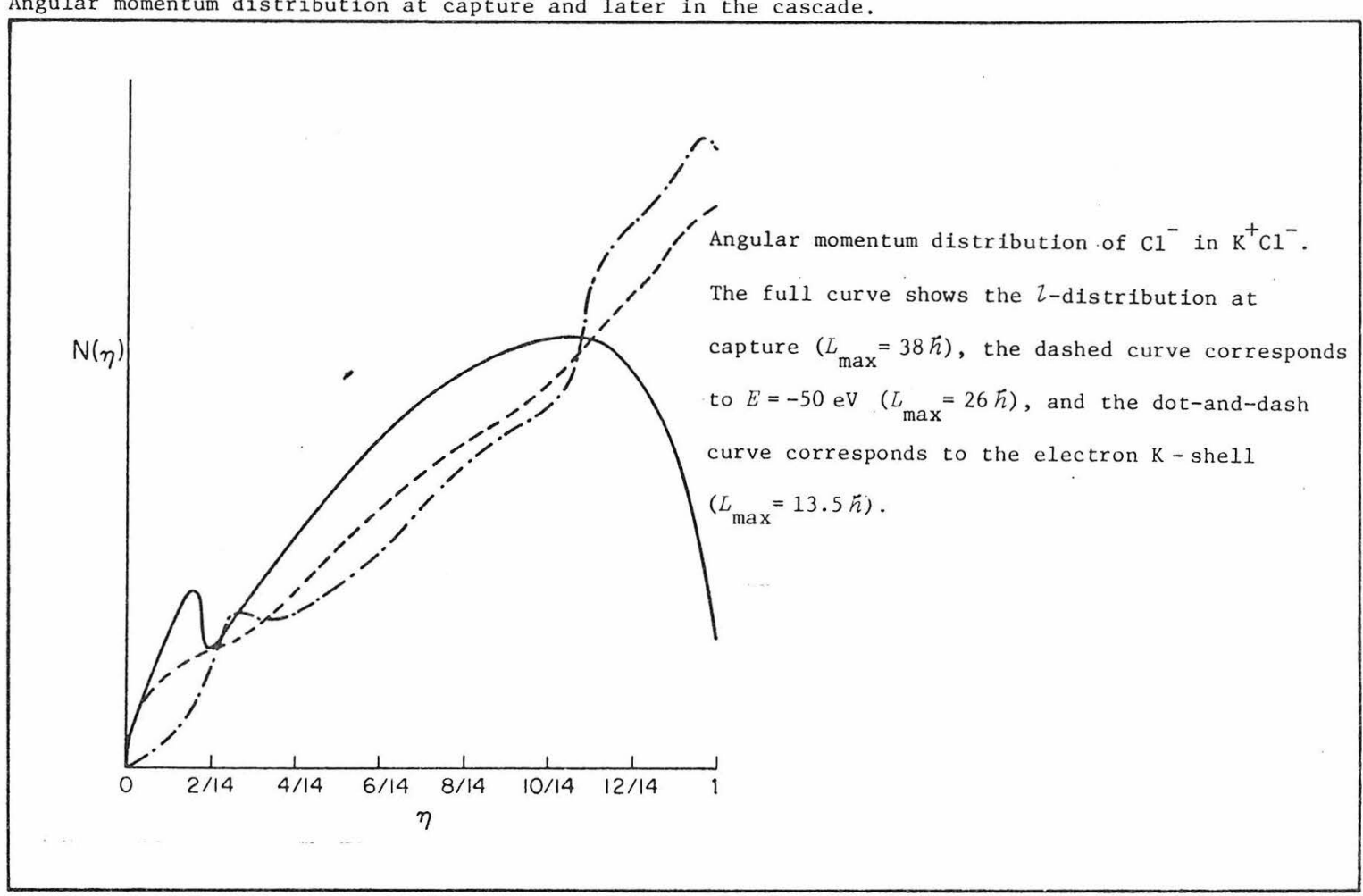

Figure 1.8

Muon spin precession angle as a function of energy and relative angular momentum.

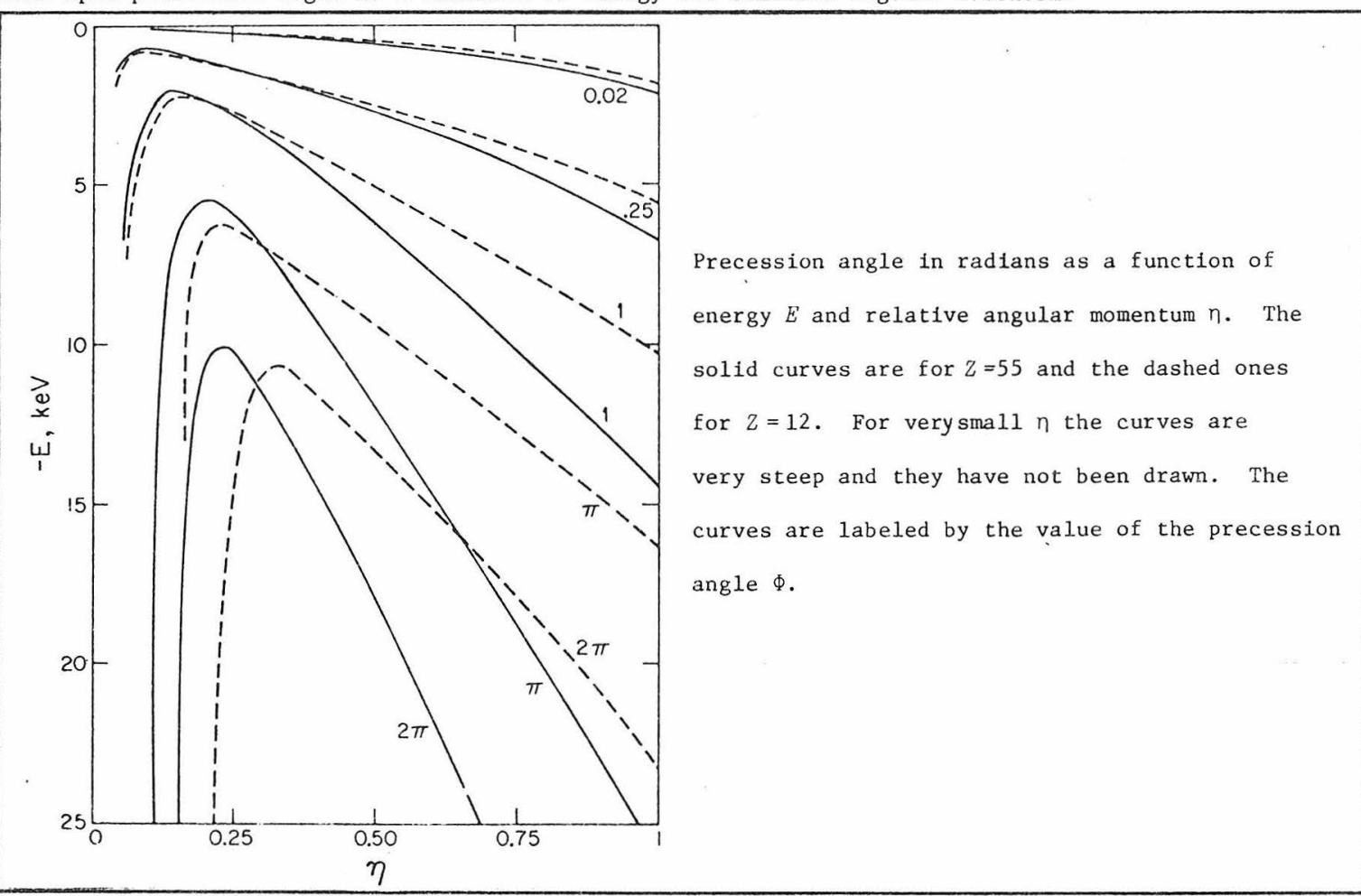


for the minimum approach distance [18], we obtain the accumulated deflection angle $\Phi$ for the spin

$$
\Phi=\frac{1}{4} \alpha^{4} \frac{\hbar}{m_{\mu} c}\left(3 \pi^{2} \rho\right)^{\frac{1}{3}} \sqrt{\frac{m_{e} e^{2}}{r \mathrm{~d} V / \mathrm{d} r}}\left\{\boldsymbol{\mu}_{e} \cdot \mu_{\mu}-3\left(\boldsymbol{\mu}_{e} \cdot \hat{\boldsymbol{r}}\right)\left(\boldsymbol{\mu}_{\mu} \cdot \hat{\boldsymbol{r}}\right)\right\},
$$

where $\rho$ is the electron (number) density and $\hat{\boldsymbol{r}}$ denotes a unit vector. The factor in braces is at most unity. Inserting a typical size of a muon orbit we get $\Phi \sim 10^{-7}-10^{-6}$ rad. This, coupled with the fact that the muon makes $10^{3}$ muon-electron collisions in the slowing down and cascade processes, shows that there is insignificant depolarization caused by this mechanism.

1.4.2. Depolarization Due to the Spin-Orbit Coupling. When the spin-orbit coupling splitting becomes larger than the line width [30], the muon spin is no longer a "good quantum number". The original polarization $P_{1 n} \equiv\left\langle\sigma_{z}\right\rangle$ is then reduced by a factor $1 / 3$. Classically one has to project the spin $s$ on the angular momentum $\boldsymbol{j}$ axis (assumed random) and back to the original spin direction. Thus the net polarization is

$$
P=P_{\text {in }} \frac{\int_{0}^{\frac{1}{2} \pi} \sin ^{2} \theta d(\sin \theta)}{\int_{0}^{\frac{1}{2} \pi} d(\sin \theta)}=\frac{1}{3} P_{\text {in }} .
$$

The analogous quantal expression is

$$
P=P_{\text {in }} \frac{(\boldsymbol{s} \cdot \boldsymbol{j})^{2}}{(\boldsymbol{s})^{2}(\boldsymbol{j})^{2}}=\frac{1}{3} P_{\text {in }}\left(1 \pm \frac{2}{2 \tau+1}\right) \text { for } j=\imath \pm \frac{1}{2} \text {. }
$$

As we shall establish below, $j$ becomes a good quantum number at relatively large values of the principal quantum number $n$; this in conjunction with the fact that the $l$-distribution is peaked at high 2 values, makes (1.26) and (1.27) practically equivalent. The large deflections experienced by the muon at each collision during the slowing down support the assumption that the direction of $l$ (and therefore $j$ ) at capture is random.

A detailed numerical calculation of the accumulated angle $\Phi$ due to the Larmor precession, given by

$$
\Phi=\frac{1}{2 m_{\mu} c} \int r(\theta) \frac{\mathrm{d} V}{\mathrm{~d} r} \mathrm{~d} \theta
$$

where the integral is taken around the orbit, has been performed. Figure 1.8 shows the iso- 2 ines as functions of the muon binding energy and relative angular momentum $\eta$. The point where $\Phi \approx 1$ determines the transition region for $j$ becoming a good quantum number. Alternatively, the comparison between the total width of the quantum state $(n, l)$ and the fine structure splitting, given by

$$
\Delta E_{\text {f.s. }}=\frac{0.15 Z}{n^{3} z(2+1)} \quad \mathrm{eV} \quad \text {, }
$$

yields $\Phi \approx 1$. Note that for different $l$ states, this takes place at different energies (or $n$ values).

1.4.3. Quantal Depolarization. In a transition $i \Rightarrow f$ of multipolarity $L$ the polarization is reduced by a factor $\beta$, i.e., $P_{f}=\beta P_{i}[30]$, where 


$$
\beta=\frac{j_{f}\left(j_{f}+1\right)-2_{f}\left(z_{f}+1\right)+3 / 4}{j_{i}\left(j_{i}+1\right)-\tau_{i}\left(l_{i}+1\right)+3 / 4} \cdot \frac{j_{i}\left(j_{i}+1\right)+j_{f}^{\left(j_{f}+1\right)-L(L+1)}}{2 j_{f}\left(j_{f}+1\right)} .
$$

This reduction has been programmed in the depolarization part of the computer program. To illustrate its use, we can analytically estimate the residual polarization of the muon at the 1 s state. By considering the strong dipole transitions only $\left(z_{f}=Z_{i}-1\right)$, assuming a statistical 2 -distribution and using an approximation for the energy where the depolarization (1.27) becomes effective, we find that

$$
P_{1 \mathrm{~s}} \approx \frac{3}{20} \frac{n_{0}+10 / 3}{n_{0}}
$$

where $n_{0}$ is the spherical orbit at which the spin-orbit splitting equals the total decay width.

Reference [30] compares our findings with other theories [32-38] and with experimental results $[39,40]$. In the cascade program, the point at which (1.27) takes effect can be externally specified and the most straightforward way of determining it is by examination of the line width and spin-orbit energy output of a preliminary run.

1.5. Semiclassical Approach. A formalism halfway between the essentially classical description of this chapter and the purely quantal one of the following chapter can be obtained by considering the muon moving in a classical (Kepler like) trajectory through the atom, while the electrons and their interaction with the muon are treated in a quantal sense. Since we are interested in muon orbits that come quite close to the nucleus (penetrate the electron $\mathrm{K}$-shell, for example), we can take exact Keplerian orbits for the muon. We can describe both positive energy (hyperbolic) and negative energy (elliptic) orbits in this approach. The probability that between time $t_{1}$ and $t_{2}$ a transition (summarized in figure 1.9) takes place, is given by the first order perturbation result

$$
P=\left|\frac{1}{\hbar} \int_{t_{1}}^{t_{2}} \mathrm{~d} t\left\langle f\left|\sum_{\substack{p \\ \text { (all electrons) }}} \frac{e^{2}}{\left|\boldsymbol{r}_{i}(t)-\boldsymbol{r}_{p}\right|}\right| i\right\rangle \exp \left[i \frac{\Delta E}{\hbar} t\right]\right|^{2} \text {. }
$$

The various multipolarities of the interaction can be extracted by expanding the operator $e^{2} /\left|\mathbf{r}_{i}(t)-r_{p}\right|$ in spherical harmonics as in the quantal cases of chapter 2 . We will not elaborate on the mathematical details of transforming (1.32) into an algebraic formula for a transition rate. As the limits of integration tend to $-\infty$ and $+\infty$ respectively, the resulting formulas for the low multipolarities and low electronic shells involve Bessel functions (modified Bessel functions) for closed (open) orbits and all integrals can be evaluated in terms of these and other elementary functions. Similarly, for a Kepler orbit the multipole radiative rates can be evaluated (this approximation is still adequate, since the orbits that radiate significantly come very close to the nucleus where the potential is essentially Coulomb like).

We have compared these results for elliptic orbits with both their classical and quantal counterparts. For the comparison with the quantal results we have examined the rate for a dipole transition of the muon $\left(n_{1}, l_{1}\right) \Rightarrow\left(n_{2}, l_{2}\right)$ and the corresponding semiclassical rate, using as an effective $n$ the value 
Figure 1.9

Schematic representation of the setup for a semiclassically treated muon Auger transition.

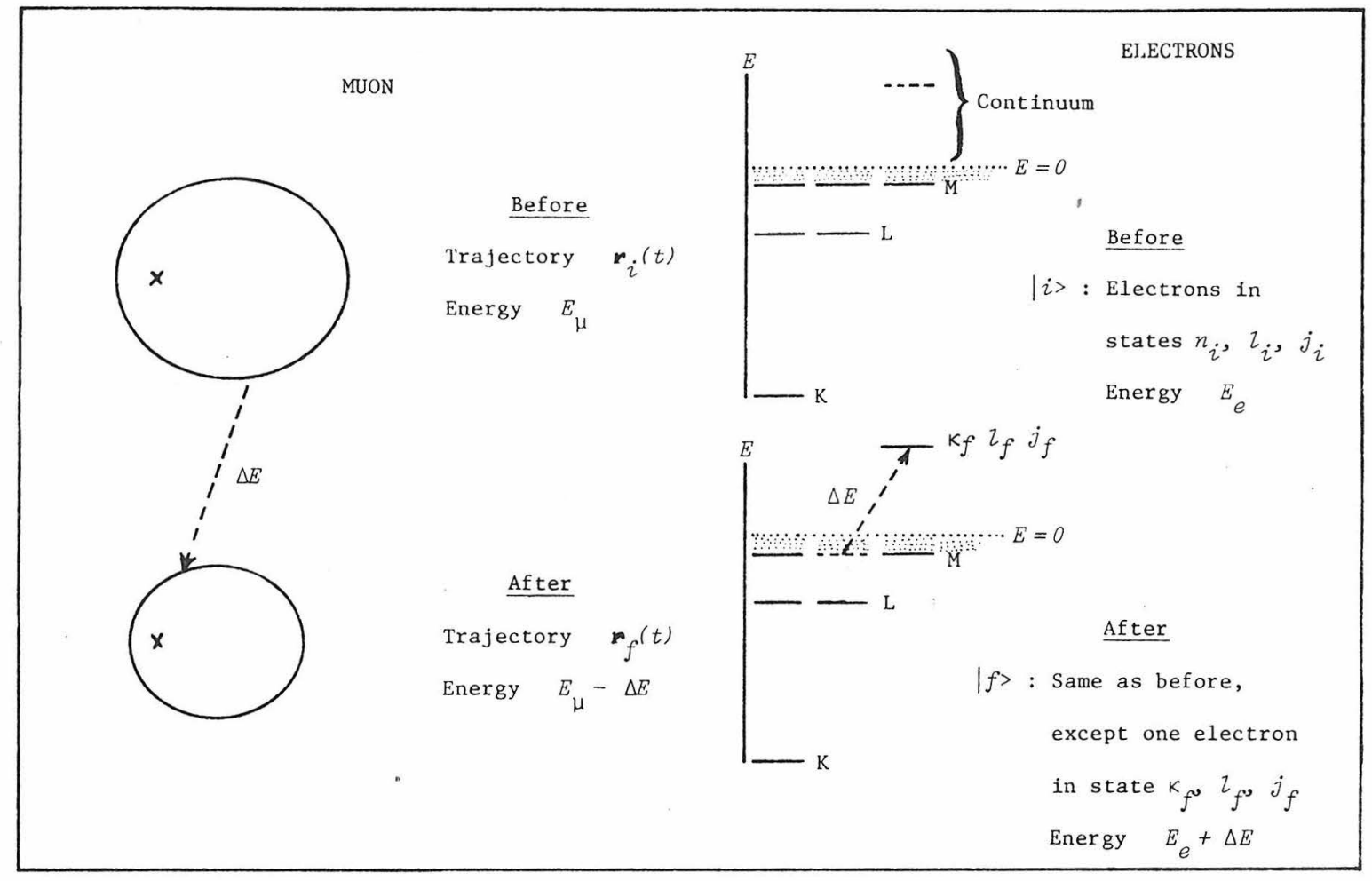

Figure 1.10

Comparison between the classical and semiclassical Auger rates.

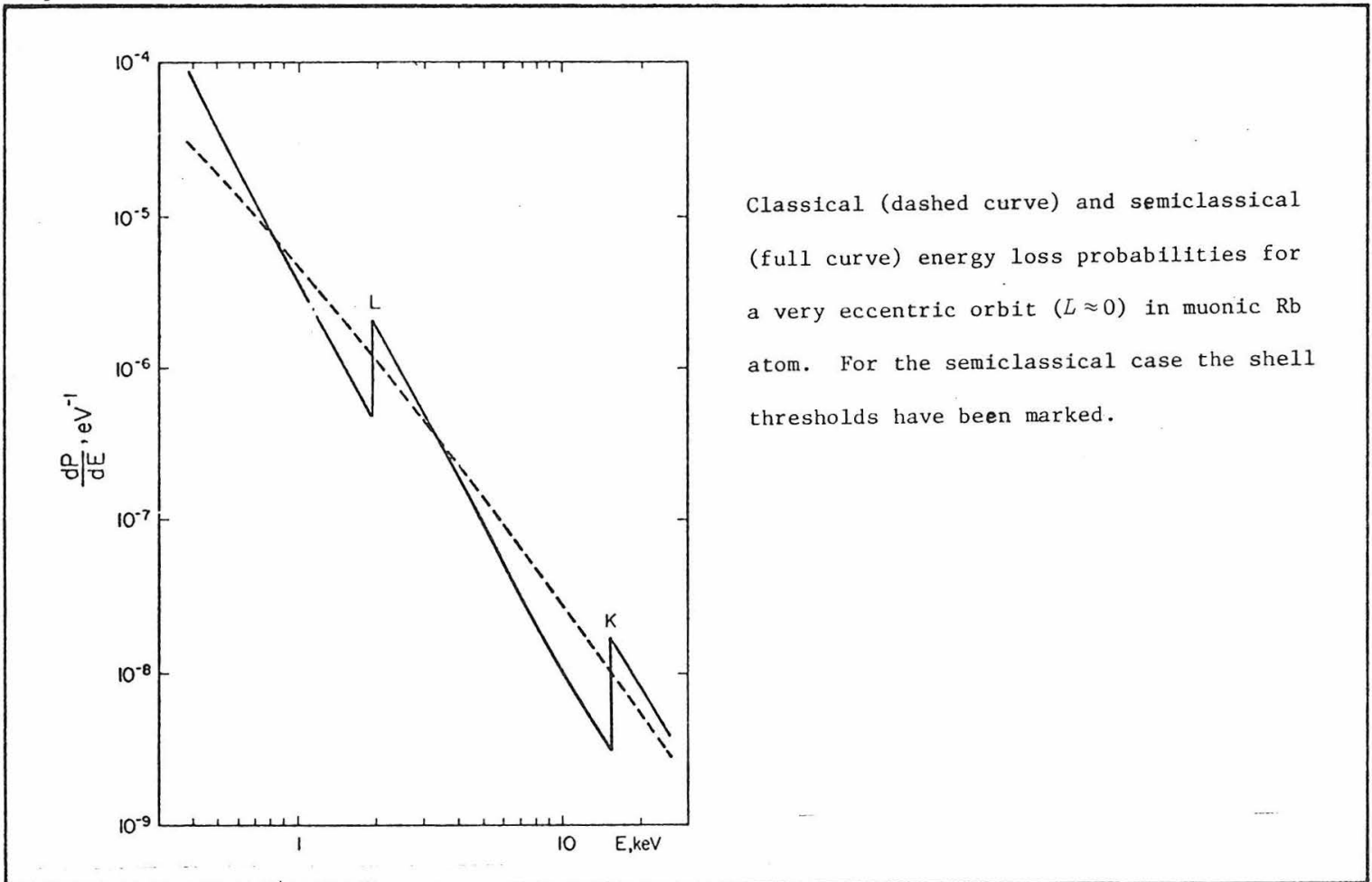




$$
n_{e}=\left(\frac{2 n_{1}^{2} n_{2}^{2}}{n_{1}+n_{2}}\right)^{\frac{1}{3}}
$$

and as an effective $Z$ the value

$$
z_{e}=\frac{1}{2}\left(z_{1}+z_{2}+1\right) \quad \text { (1.34) }
$$

For transitions with all quantum numbers large the differences are less than $5 \%$ (often less than $1 \%$ ).

For large $n_{1}-n_{2}$ (and therefore $l_{1}, l_{2}$ small) there is a marked deviation (up to factors of $3-5$ ), the quantal rates being systematically smaller. For the comparison with the classical rates, we can examine these rates as a function of energy transfer to the electron. The probabilistic formulation has been used for the classical results. Figure 1.10 has the comparison of two such typical cases. The agreement is perfect, considering the fact that the semiclassical rate exhibits threshold effects at the binding energies of the corresponding shells. It is believed that at small energy losses ( $\sim$ a few eV) the classical formalism is superior. No such comparison can be readily made, because the wave functions of the valence electrons are in no acceptable approximation Coulomb like.

The findings of this section have bridged the gap between a punely classical and a purely quantal description and the agreement of all three theories at their overlapping regions reassures us that a smooth transition of formalism could be attained without severe change of the quantities involved. 
CHAPTER 2: QUANTAL CASCADE AND TRANSITION RATES

Armed with the results of the previous chapter and having an approximate idea of the distribution of muons at the energies where the transition from classical to quantal description occurs, we embark on the task of describing the subsequent energy loss and depolarization mechanisms in a full quantal regime. The pioneer work of references [41-44] has been of great help, as we have sifted through the various possibilities to establish which phenomena can be safely neglected and which can be markedly improved. Appendix A contains most of the mathematical details and a compilation of formulas involved in this development.

2.1. Overview of the Quantal Mechanisms. The general approach of a quantal calculation of transition rates that cannot be solved exactly, is in the framework of perturbation theory. The justification of the implementation of this method can be validated by computing higher order corrections and showing that they are indeed small. In the following we will restrict ourselves to the lowest (nonvanishing) order terms in any perturbation series expansion.

The fundamental formula used in relation to any transition rate between an initial state $i$ and a final state $f$ is the Fermi golden rule, giving the reciprocal life time of such a transition

$$
\frac{1}{\tau}=\frac{2 \pi}{\hbar} \oint\left|\mathscr{H}_{f i}\right|^{2} \frac{\mathrm{d} N(E)}{\mathrm{d} E}
$$

where $\mathscr{H}_{f i}$ is the matrix element of the perturbation Hamiltonian operator, $\mathrm{d} N(E) / \mathrm{d} E$ is the density in energy of the final states available, and $S$ denotes a summation over propagation directions, polarization states, and magnetic quantum numbers (or other possible unobserved parameters). Note that most of the problem is concentrated in evaluating the matrix element of the perturbation Hamiltonian

In both radiative and Auger transitions, it is useful to expand the interaction Hamiltonian into multipole terms. This is a mathematically general expansion in principle, but in our case, where $k r \ll 1$ ( $k$ is a typical wave number in the problem, derived, say, from the energy transfer, and $r$ ranges over the muonic orbit dimensions), such an expansion converges quite rapidly. Moreover, the various multipole parts connect initial and final states according to specific selection rules of the quantum numbers; as a result for small quantum numbers, only a limited number of multipolarities is allowed in any case. In the following we will discuss in detail the two principal modes of energy loss and their implications on the cascade.

2.2. Emission of Multipole Radiation. In figure 2.1 we show the transition of a muon between the two states indicated, all taking place in the Coulomb field created by the atom. The lowest order interaction Hamiltonian is given in the non-relativistic approximation by [45]

$$
\mathscr{H}(\boldsymbol{A})=\frac{e}{m_{\mu} c} \frac{\boldsymbol{p} \cdot \boldsymbol{A}+\boldsymbol{A} \cdot \boldsymbol{p}}{2}-\mu_{\mu} \frac{e \hbar}{2 m_{\mu} c} \boldsymbol{\sigma} \cdot \boldsymbol{H},
$$

where $\boldsymbol{A}$ is the vector potential, $\boldsymbol{H}$ is the magnetic field, $\boldsymbol{\sigma}$ is the Pauli spin vector and $\boldsymbol{p}$ is the momentum operator. Furthermore, expanding in multipoles and separating the electric type terms from the magnetic type terms one obtains for the transition rates in the long wave length approximation 


$$
\Gamma_{i \rightarrow f}^{\sigma L M} \equiv\left[\frac{1}{\tau}\right]_{i \rightarrow f}^{\sigma L M}=\frac{8 \pi(L+1)}{L[(2 L+1) ! !]^{2}} \frac{1}{\hbar}\left(\frac{\omega}{c}\right)^{2 L+1}\left|\left\langle f\left|\mathcal{M}_{\mathrm{LM}}^{\sigma}\right| i\right\rangle\right|^{2}
$$

where the "reduced" multipole operators $\mathscr{M}_{L M}^{\sigma}$ are given by

$$
\begin{aligned}
& \mathcal{M}_{L M}^{E}=e r^{L} Y_{L M}^{*}-i \frac{\mu_{\mu} e \hbar \omega}{2 m_{\mu} c^{2}} \frac{1}{L+1}(\boldsymbol{\sigma} \times \boldsymbol{r}) \cdot\left[\nabla\left(r^{L} Y_{L M}\right)\right]^{*}, \\
& \mathcal{M}_{L M}^{M}=\frac{e \hbar}{m_{\mu} c} \frac{1}{L+1} L \cdot\left[\nabla\left(r^{L} Y_{L M}\right)\right]^{*}+\frac{e \hbar}{2 m_{\mu}{ }^{c}} \mu_{\mu} \cdot \cdot\left[\nabla\left(r^{L} Y_{L M}\right)\right]^{*} .
\end{aligned}
$$

Note that the second term in each expression (2.4) arises from the muon spin.

For $E(L)$ transitions the spin term is generally small, and if one neglects it completely, the formulas of Appendix sect. A.l can be readily obtained. For the magnetic type transitions, it turns out that all radial dependence drops out for $L=1$ (due to the fact that $\nabla\left(r Y{ }_{1 M}\right.$ ) is not a function of $r)$. But in such a case, the transition rate between states of different $n$ vanishes, due to the orthogonality of the radial parts of the wave functions. This means that $M 1$ transitions in this approximation can go only between different fine structure components (such transitions are of no particular interest to us). However, higher $M(L)$ transitions can connect different $n$ states, the intensity of such lines being exceedingly small.

As a digression, we can estimate the relativistic corrections to the $M 1$ operator, which indeed provides some rate between different $n$ states, due to its $r$ dependence. More specifically, the non-relativistic operator

$$
\left.\mathcal{M}_{1 \mathrm{M}}^{\mathrm{M}}\right)_{\mathrm{n} \cdot \mathrm{r} .}=\frac{e \hbar}{2 m_{\mu} c}\left(\boldsymbol{L}+\mu_{\mu} \approx\right) \cdot\left[\nabla\left(r Y_{1 M}\right)\right]^{*},
$$

becomes with the first order relativistic corrections [46]

$$
\left.\mathcal{M}_{1 M}^{M}\right)_{\operatorname{Re} 1}=\frac{e \hbar}{2 m_{\mu} c}\left\{1+\frac{1}{10}\left(\frac{\omega}{c}\right)^{2} r^{2}+\frac{1}{2} \frac{p^{2}}{m_{\mu}^{2} c^{2}}+\cdots\right\}\left(L+\mu_{\mu} \sigma\right) \cdot\left[\nabla\left(r Y_{1 M}\right)\right]^{*} .
$$

As an order of magnitude estimate (for $n_{1}-n_{2} \ll n_{1}, n_{2} \approx n$ ) we can say that

$$
\left.\left\langle M_{1 M}^{M}\right)_{\operatorname{Re} 1}\right\rangle_{n_{1} \neq n_{2}} \simeq \frac{e \hbar}{m_{\mu} c} \frac{(Z \alpha)^{2}}{n} .
$$

We will later see how this compares to the electric multipole rates.

2.3. Energy Loss via Ejection of Atomic Electrons (Auger Transitions). Whereas radiation results in the emission of a free photon, while the muon is making a quantal transition in the field of the atomic potential, this photon can be virtual and interact with an atomic electron and get absorbed by i.t, giving rise to an electron with increased energy. This second order process, depicted in figure 2.2, not only cannot be neglected (as higher order), but under certain conditions it is the predominant channel of energy loss in a transition between two states. This process is, of course, nothing else than a quantal picture of the muon-electron collisions, described classically in chapter 2 . The 
Figure 2.1

Schematic representation of a radiative muon transition.

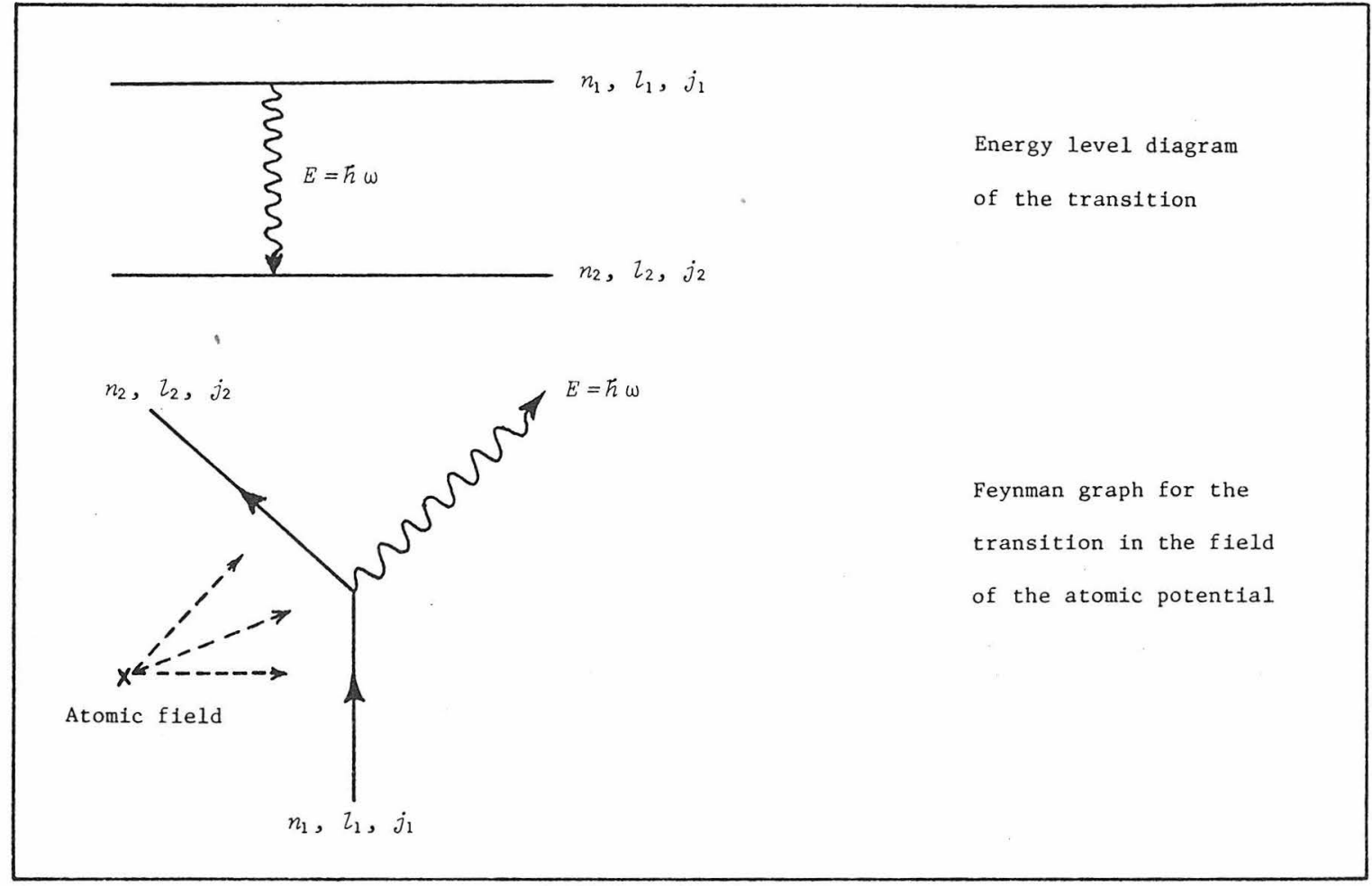

Figure 2.2

Schematic representation of a muon Auger transition.

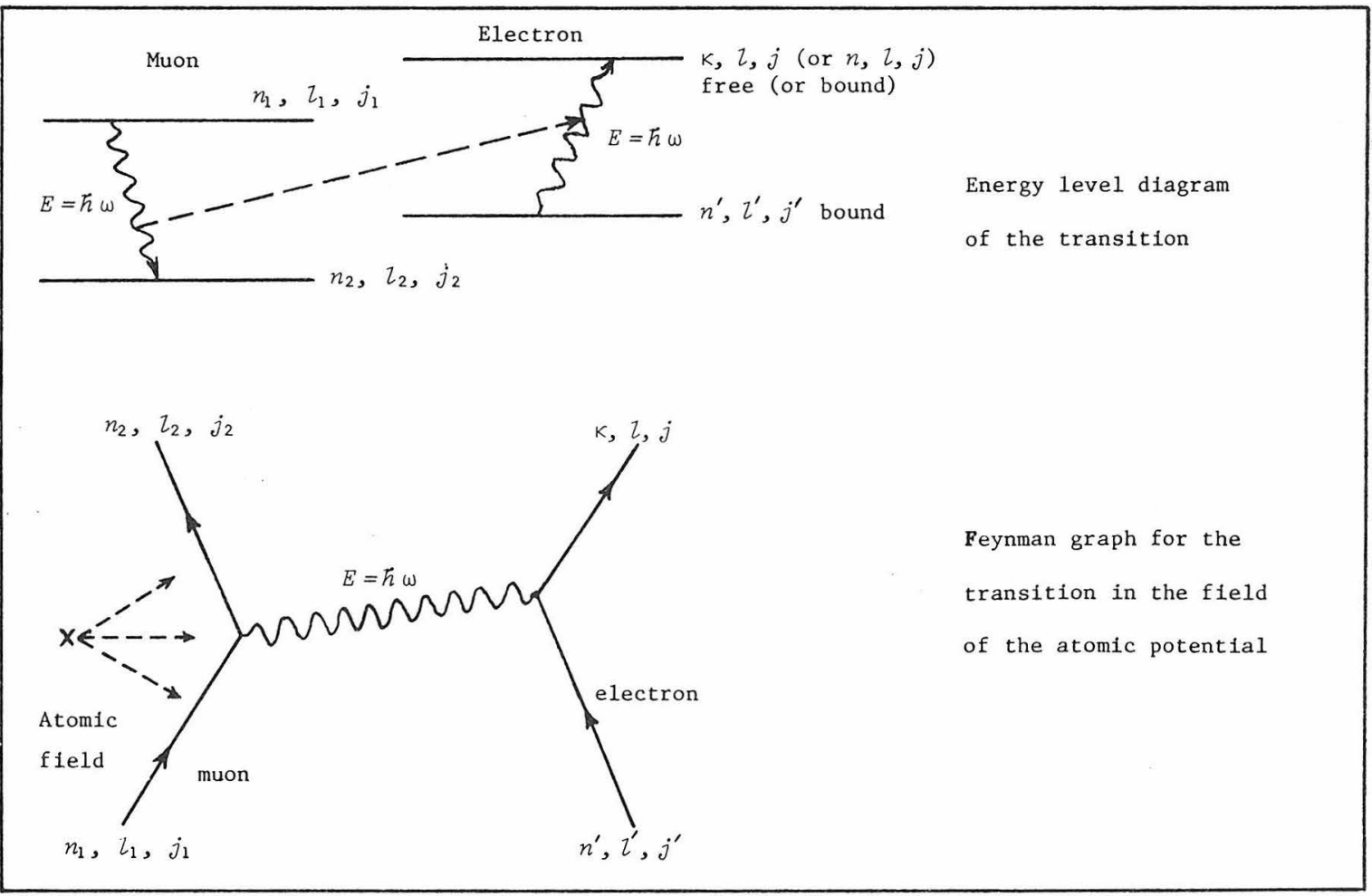


interaction Hamiltonian in a non-relativistic approximation is

$$
\mathscr{H}_{12}=e^{2} \frac{\mathrm{e}^{-i K r_{12}}}{r_{12}}+\text { Angular momentum related parts } \text {. }
$$

It turns out that we can safely limit ourselves in the treatment of the electric part of the interaction indicated; an order of magnitude estimate for the magnetic type transition rates will follow later. Section A.3 has the mathematical development of the rate formulas. One rnust realize that a considerable amount of effort has been devoted in the calculation and verification of these expressions.

2.4. Improvements on the Transition Rates. Whereas the formulation of the radiation is simple, straightforward, and quite reliable, the same is not even remotely true for the Auger transitions. The only possible flaws in the radiation are the use of simplified wave functions for the muon and the neglect of the spin-related terms in the Hamiltonian. On the other hand, the Auger transitions suffer from the electronic wave functions, which are more crude, and a host of other inaccuracies. We have investigated the most obvious ones, arriving at some answers. Following are some of the most important corrections.

2.4.1. Retardation. In a simple treatment one omits the exponential factor in eq. 2.8, pretending that the interaction is mediated by a photon of a range that is much shorter than its wave length. This is, of course, the limit of a four point interaction, where the ejection of the electron occurs "simultaneously" with the change of levels of the muon. The exponential retardation factor, as a direct result of relativity, provides for the necessary oscillations of the photon field until it has been absorbed in the other vertex. As expected, the correction should be substantial for high energy transitions; figure 2.3 shows the modification of the 1 s and $2 \mathrm{~s}$ rates as a function of energy, with this refinement. Since the onset of any significant deviation from unity occurs at energies $\hbar \omega \geqslant \frac{1}{2} m e_{e} c^{2}$, where as we will see, Auger rates are small, this correction can be retained for academic purposes only, and in fact it has not been implemented in the computer program.

2.4.2. Electronic Screening. Ideally one would like to solve the full atomic potential with a muon in a particular state, in order to determine the modified eigenfunctions of the many body problem. This task is conceivably possible in a Hartree-Fock self consistent manner; since this exceeds the scope of this thesis, we can only study the perturbation of the muon on the atomic wave functions in a phenomenological fashion. Guided by classical considerations $[24,25,26$, and private work unpublished] of the "bubble" formed around the muon (i.e., the rarefaction of the electronic cloud in the proximity of the muon), we can simulate this reduction of the electromagnetic interaction by introducing a Yukawa type multiplicand in the expression of the Hamiltonian. Section A.4 has some details on this; the impact of this refinement is discussed in section 2.5.

2.4.3. Penetration. This is not a conceptual correction per se, but rather the result of a mathematical complication, which, if neglected, leads to simplified expressions. This effect is related to the fact that the electron orbits lie partially inside the muon orbits. On the other hand, 
Figure 2.3

Effect of relativistic retardation on the Auger rates.

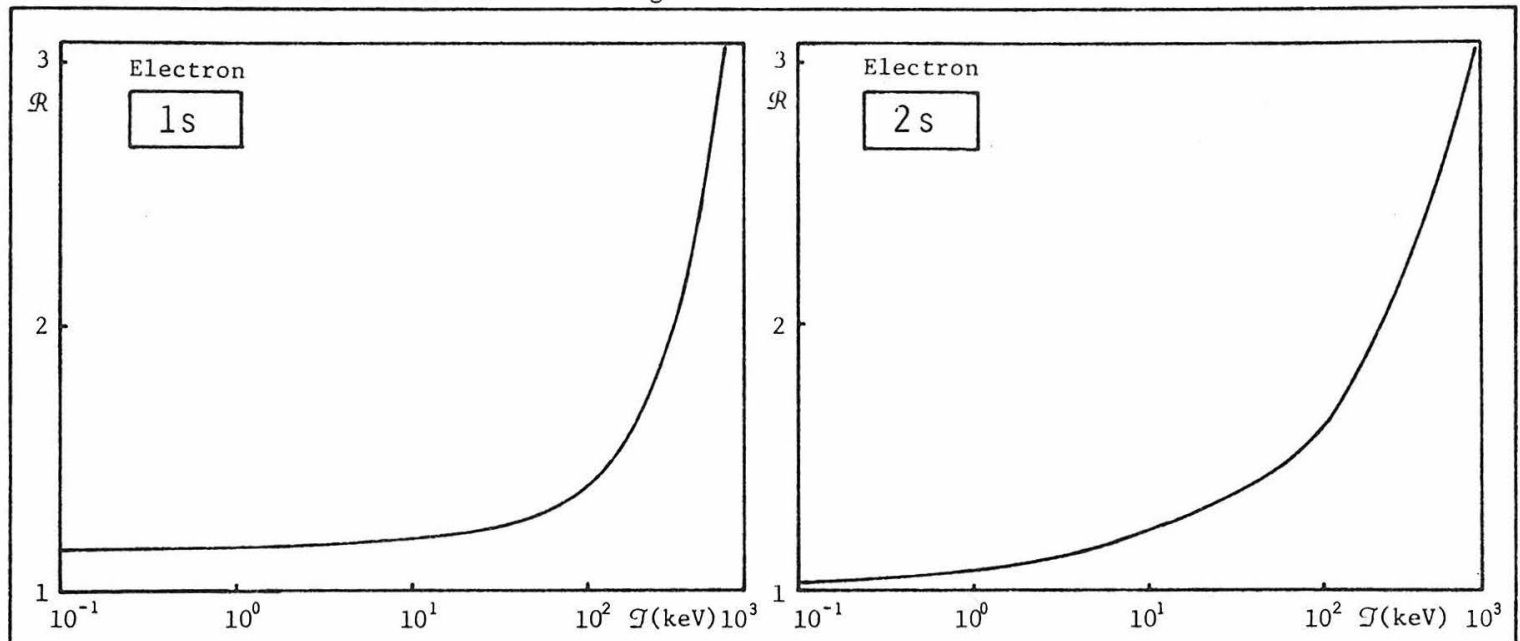

Plot of the ratio $R$ of the rate with retardation to the rate without retardation versus the kinetic energy $I$ of the continuum electron. Note that due to the non-uniform convergence of these functions at the limit $I \Rightarrow 0, R$ does not tend to unity, but to the following limits:

$$
\lim _{\mathfrak{I} \Rightarrow 0} R_{1 \mathrm{~s}}=\left[\frac{4}{4+\zeta}\right]^{2} \exp \left\{\frac{4 \zeta}{4+\zeta}\right\}, \quad \lim _{\mathfrak{I} \Rightarrow 0} R_{2 s}=\frac{(1-\zeta)^{2}}{(1+\zeta)^{4}} \exp \left\{\frac{8 \zeta}{1+\zeta}\right\} ; \quad \zeta=\left(Z^{*} \alpha\right)^{2}
$$

Figure 2.4

Schematic representation of the strong dipole radiative muon transitions in the cascade.

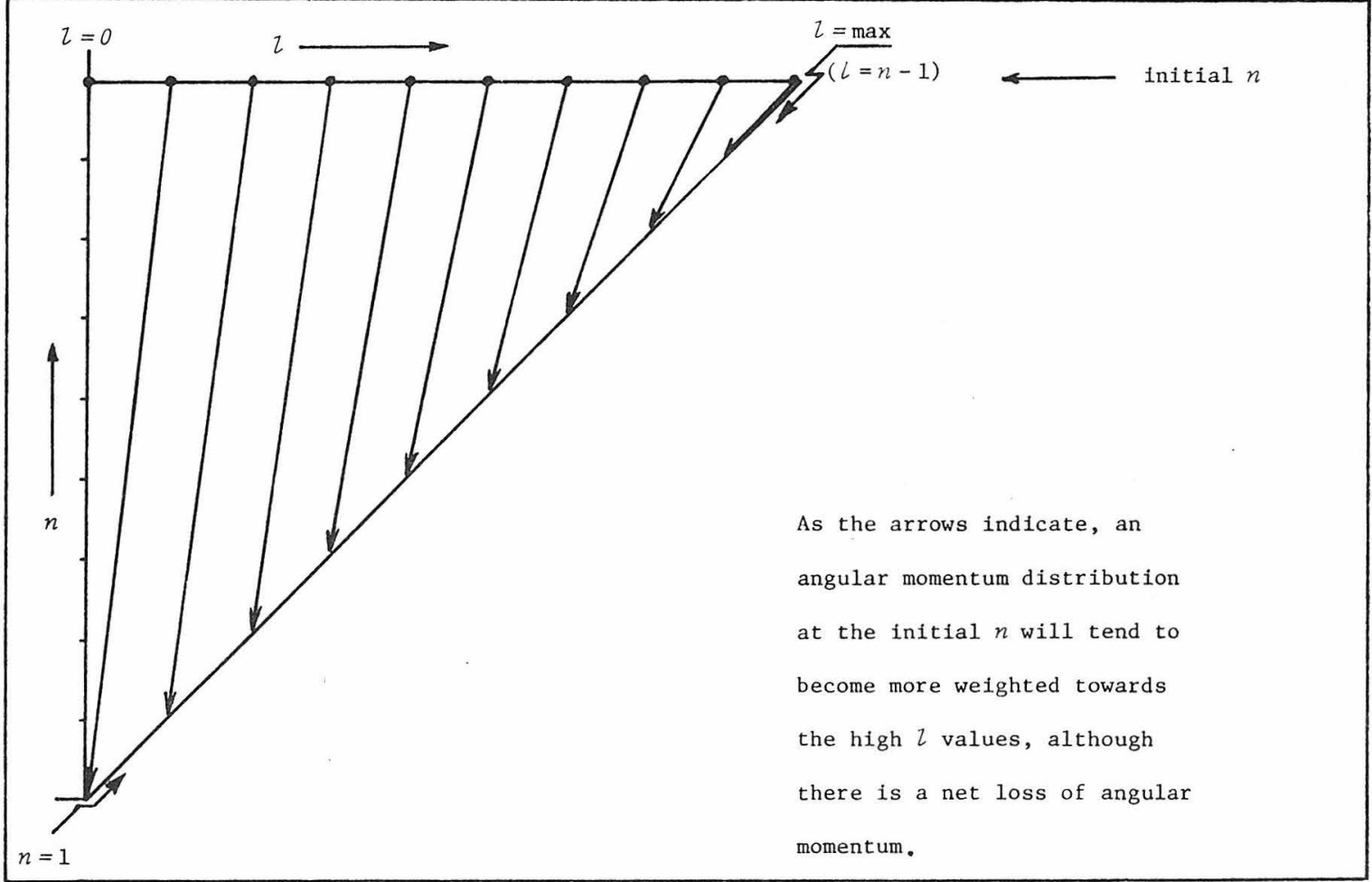


these simplifications are of limited utility, since the corrections are in the majority of interesting cases more sizable than the original terms. Section A.5 gives a comprehensive analysis of this computational effect, including a step by step description of our effort to find a solution that is numerically acceptable and computationally efficient. Unfortunately, to list a detailed table of fitted coefficients and the accompanying analytical formulas would be rather lengthy and of special interest only. The reader can recover these results from the code of the cascade program.

2.5. Relative Importance of the Various Rates - Effects of Corrections. Up to now we have examined only half of the problem; the rates and their corrections. The other half is the examination of these results in order to solidify our understanding of the underlying principles and, from the practical point of view, to be able to distinguish which rates are large, and thus have to be computed more accurately, and which are small and can be safely neglected. Following is a systematic discussion of the sizes of rates.

2.5.1. Radiation Multipoles. Monopole radiation does not exist, as exemplified by the fact that the radial parts of hydrogen like wave functions are orthogonal. The relative intensity of the various multipoles is found by using the order of magnitude estimates for the multipole matrix elements

$$
\begin{aligned}
& \left|\left\langle i\left|x^{L}\right| f\right\rangle\right| \sim \frac{n^{2 L}}{(Z \alpha)^{L}} \quad\left(n \sim n_{1}, n_{2}\right), \\
& \omega \sim(Z \alpha)^{2} / n^{2} \quad .
\end{aligned}
$$

Then apart from factors of the order of unity, the ratio between two consecutive multipoles is roughly

$$
\frac{\Gamma_{\mathrm{R}}^{\mathrm{L}+1}}{\Gamma_{\mathrm{R}}^{\mathrm{L}}} \leqslant \frac{(Z \alpha)^{2}}{n^{2}} \ll 1
$$

In addition a similar rough estimate yields fron eq. 2.7

$$
\frac{\Gamma_{R}(M 1)}{\Gamma_{R}(E 1)} \sim \frac{(Z \alpha)^{6}}{n^{6}} .
$$

The moral of this is the well known finding in atomic physics that the higher multipoles fall off quite rapidly (for very small quantum numbers such high multipoles are not allowed). Experimentally transitions up to $E 2$ have been observed [47,48] for high $Z$ atoms. To be on the safe side we have included in our calculations terms up to $E 3$. Note that for all practical purposes magnetic transitions are non existent.

The second comparison of intensities may be done within the same multipolarity and initial state, but with different final states. The strong dependence of the rates is in the transition frequency $\omega$, the rates being proportional to $\omega^{2 L+l}$. Therefore, other things being equal, the transition with the smallest $n_{2}$ is the most intense. This means that radiation tends to transfer the muon to the lowest available energy state. Finally, with the same initial state and final $n_{2}$, the transition which has $Z_{2}=l_{1}-l_{1}$ (if possible) is the strongest (an argunent about matching, the number of nodes of the radial parts of the initial and final wave functions is the reason here), the transition(s) with larger $I_{2}$ being progressively less intense. Putting all three comparisons together we can assert that 
Figure 2.5

Comparison of the relative intensity of the multipole Auger electron transitions.

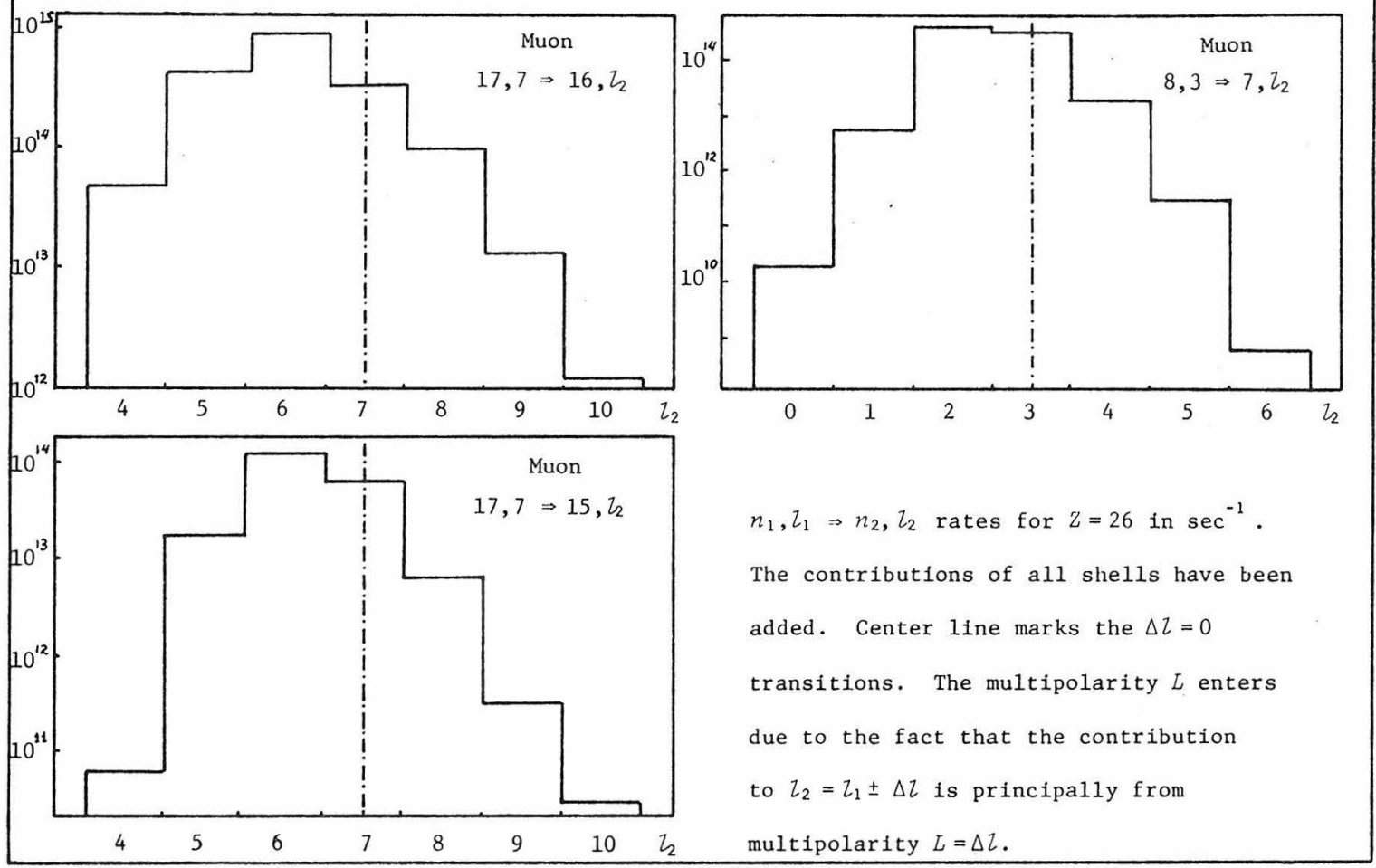

Figure 2.6

Examples of total conversion coefficients in the cascade.

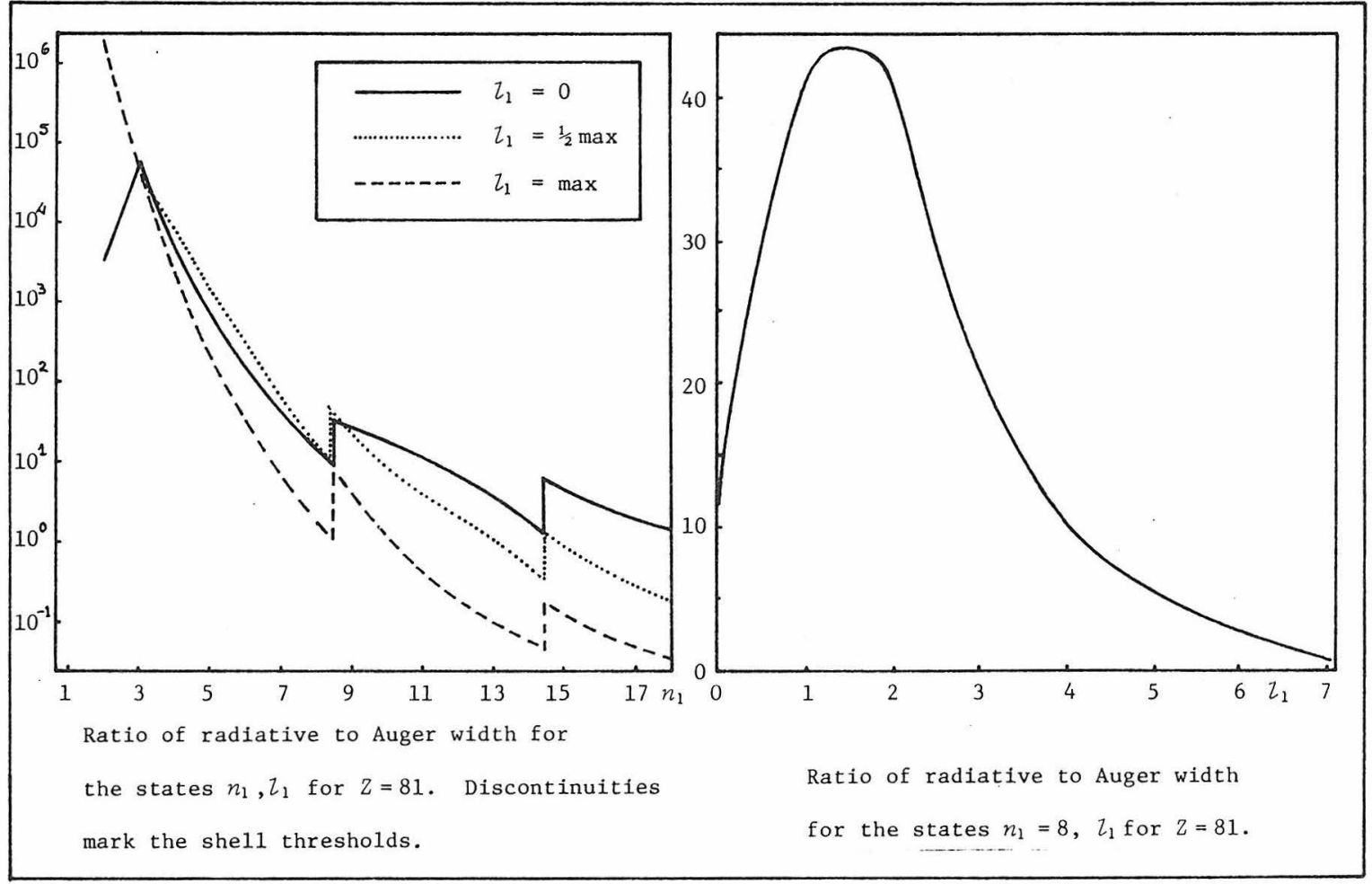


radiation tends to push the distribution of muons towards the high $Z$ states; figure 2.4 shows schematically a typical cascade situation with the strong transitions from the initial level $n_{1}$ indicated. Although the angular momentum decreases, the relative angular momentum does increase, due to the larger jumps in $n$. Whenever radiation is predominant, this picture is applicable. In the following subsection we will see the quite unsimilar features of the Auger rates.

2.5.2. Auger Rates. The Auger rates decrease with increasing multipolarity much slower than the radiative rates. In most cases, the strong dipole transition is indeed the strongest, but this is not all of the story; $E 0$ and $E 2$ transitions compete and cannot be safely neglected. Octupole and higher ones can be disregarded with no consequences; equally, the $M 1$ Auger transitions are small since $M 1$ radiation is down (eq. 2.10) and the conversion coefficients (ratio of Auger rate to radiation for the same muonic transition) are close to the $E 2$ rates [49]. Figure 2.5 demonstrates three typical cases of the comparison of the multipolarities. Examination of these rates and the radiative rates confirms the fact that the conversion coefficient increases with increasing order of the multipolarity [50].

Within each multipolarity it is instructive to examine the contribution of each shell and subshell (even each unresolved continuum $Z$ ) to the total width. The rule of thumb - found empirically and in general agreement with the formulas - is that the shell closest to threshold, i.e., the one with highest binding energy, and still allowed to go to the continuum) contributes the most, the ratio of intensities of consecutive shells being in the range 5 - 10. However, unlike radiation, Auger transition intensities are maximal for the smallest change of energy (provided that a particular subshell is open). This phenomenon counterbalances the effect of displacing the muons towards the high angular momentum states caused by the radiation. The total conversion coefficient for an initial state is usually larger than unity for high $n_{1}$ and trivially small for $n_{1} \sim 1$. Figure 2.6 shows this ratio for some specific cases; also figure 2.7 depicts the ratios for the contribution of shells to the Auger width as a function of transition energy. Note that in figs. $2.5,2.6$, and 2.7 penetration has been included. There is a wide variation of values as the quantities change rapidly with $y$. Figure 2.8 shows the energy and $y$ dependence of the dipole $x, L$, and $M$ conversion coefficients; note the similarities of the curves. Finally table 2.1 shows the ratios of the contributions of the various portions (subshells and continuum $Z$ states) at threshold - this test is not fair, since at most one shell can be very close to threshold.

2.5.3. Corrections -- Penetration. Since we have seen that the Auger transitions are not particularly important for small $n_{2}$, or equally for large $\Delta n$, we can safely disregard the relativistic retardation. Also we can dispose of the electronic screening. As a demonstration, we show in figure 2.9 the modification of the monopole operator in the presence of an unrealistically strong screening. On the other hand, penetration is quite important; to pinpoint the exact size of it is rather hard: only a few general remarks can be made. a) Penetration is most important for high muon principal quantum numbers; for $n_{1} \leqslant 5$ it is insignificant by itself and by the fact that radiation is overwhelming. b) Penetration is a necessity for monopole transitions and cannot be a priori 
Figure 2.7

Dependence of the electron Auger rates on energy and electronic shell.

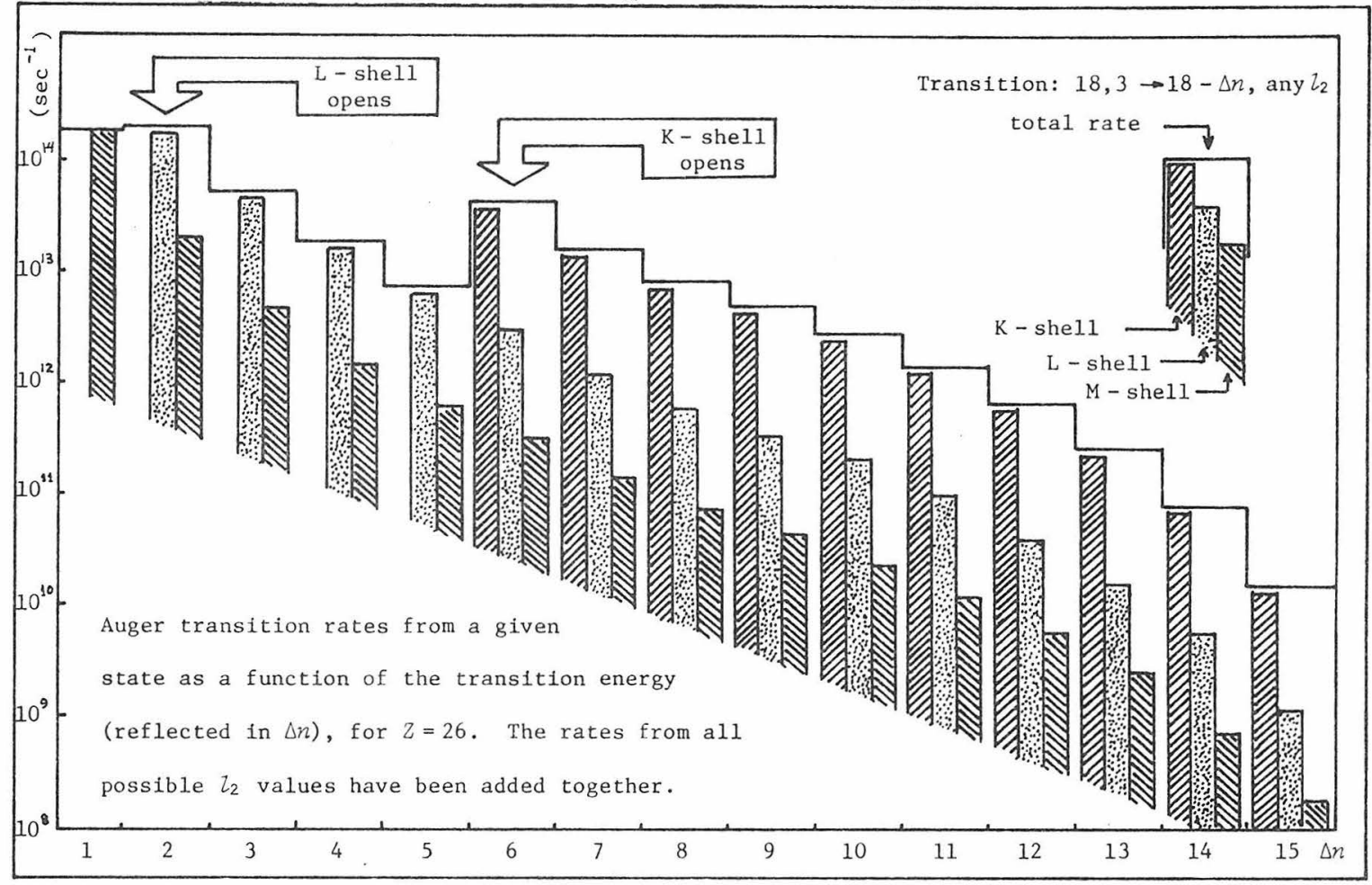

Figure 2.8

Dependence of the conversion coefficient on energy and $y$ variable.

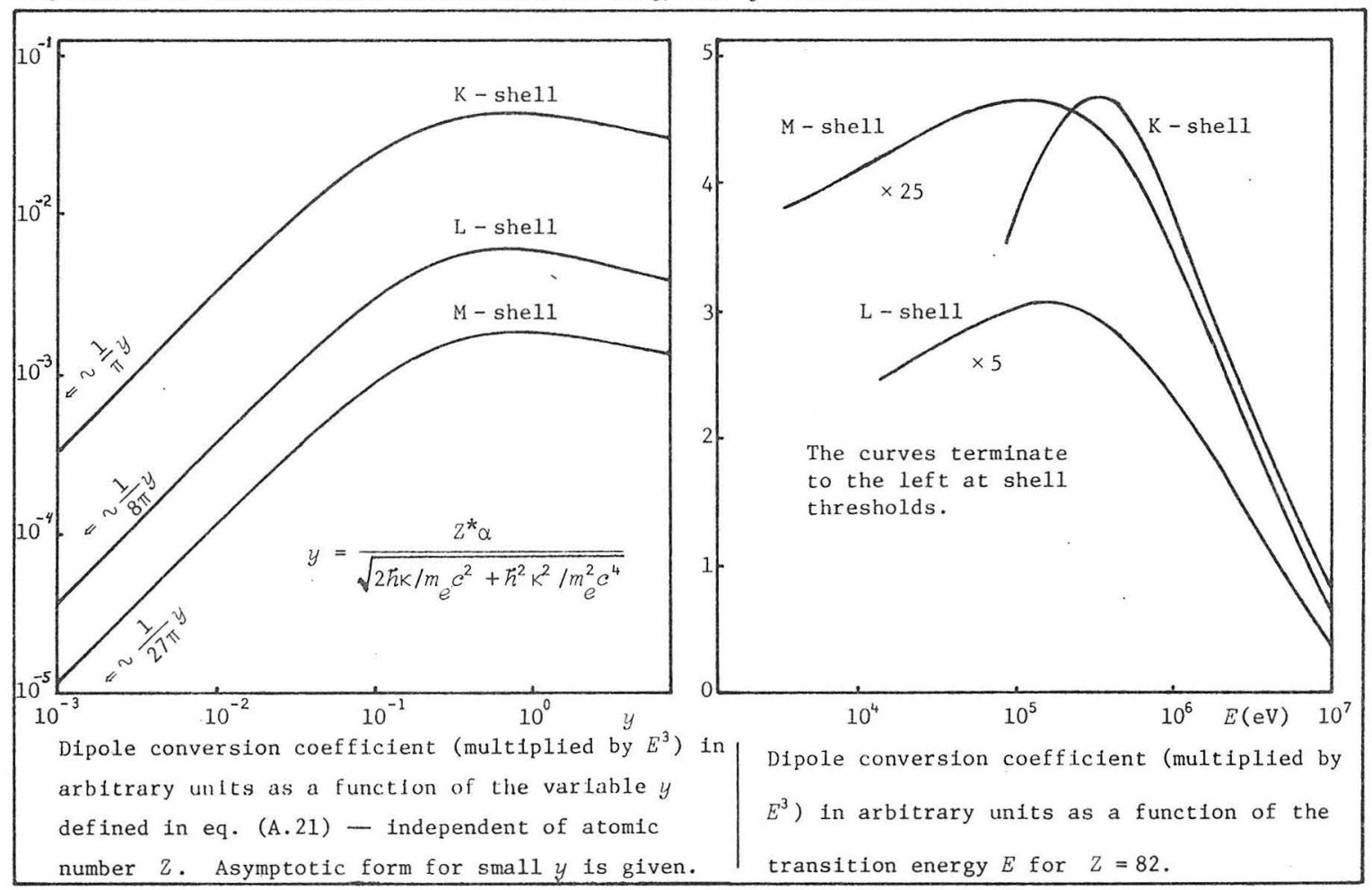




\section{$-30-$}

Figure 2.9

Modification of the monopole operator due to electronic screening.

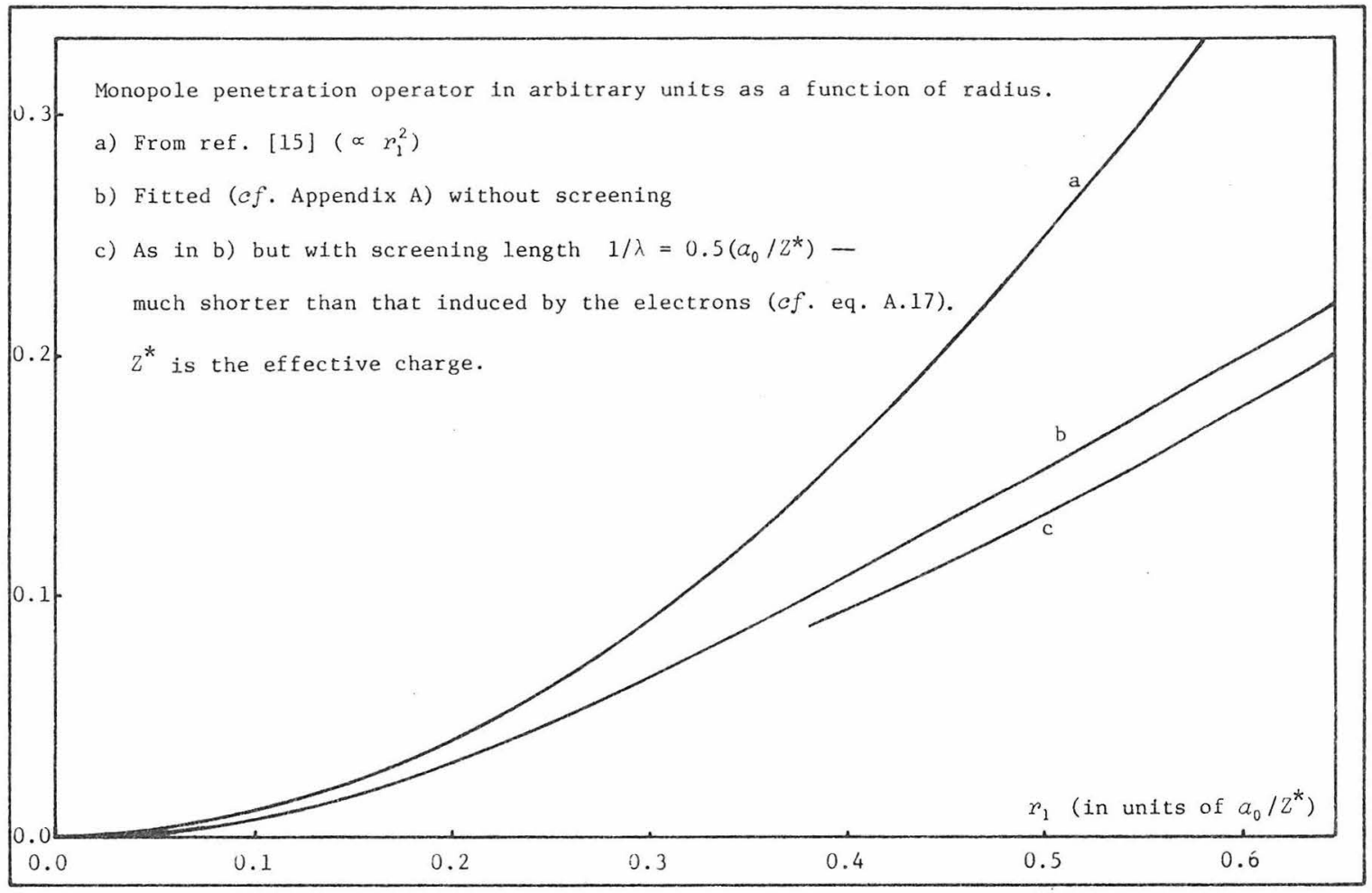

Figure 2.10

Effects of penetration on electron Auger rates.

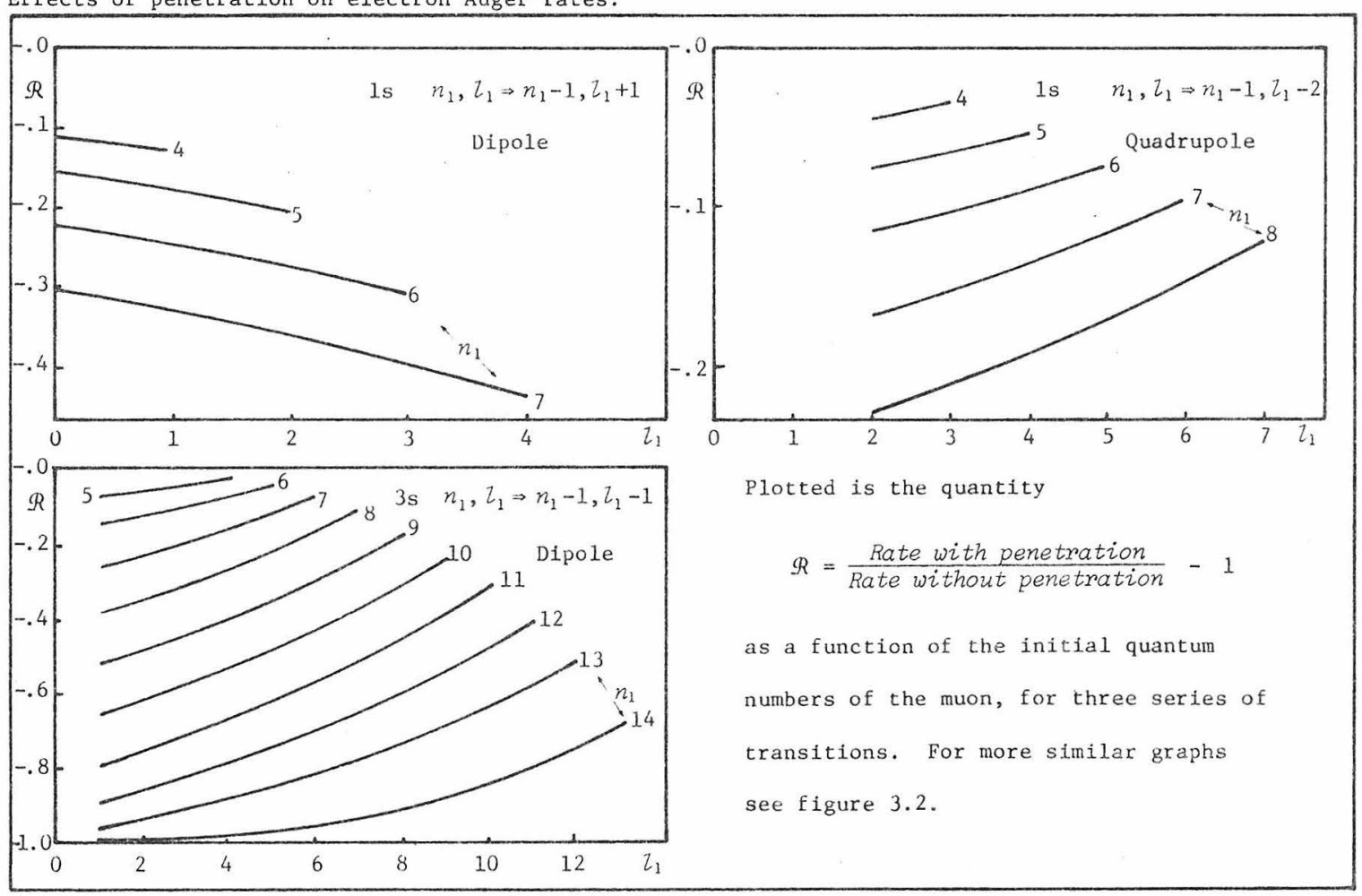


Derived fit for the effective nuclear charges.

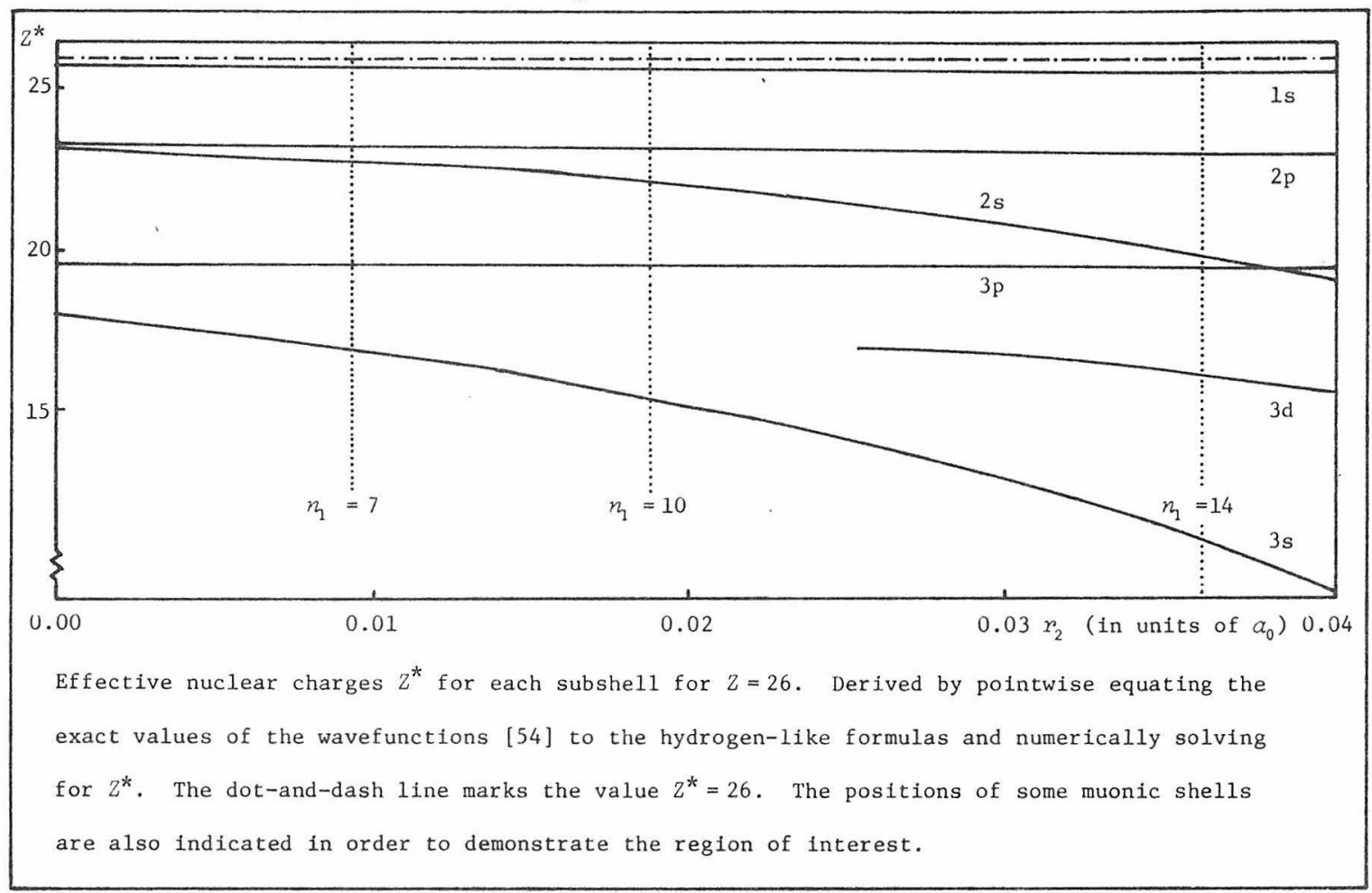

TABLE 2.1

Relative contributions of electronic shells at threshold.

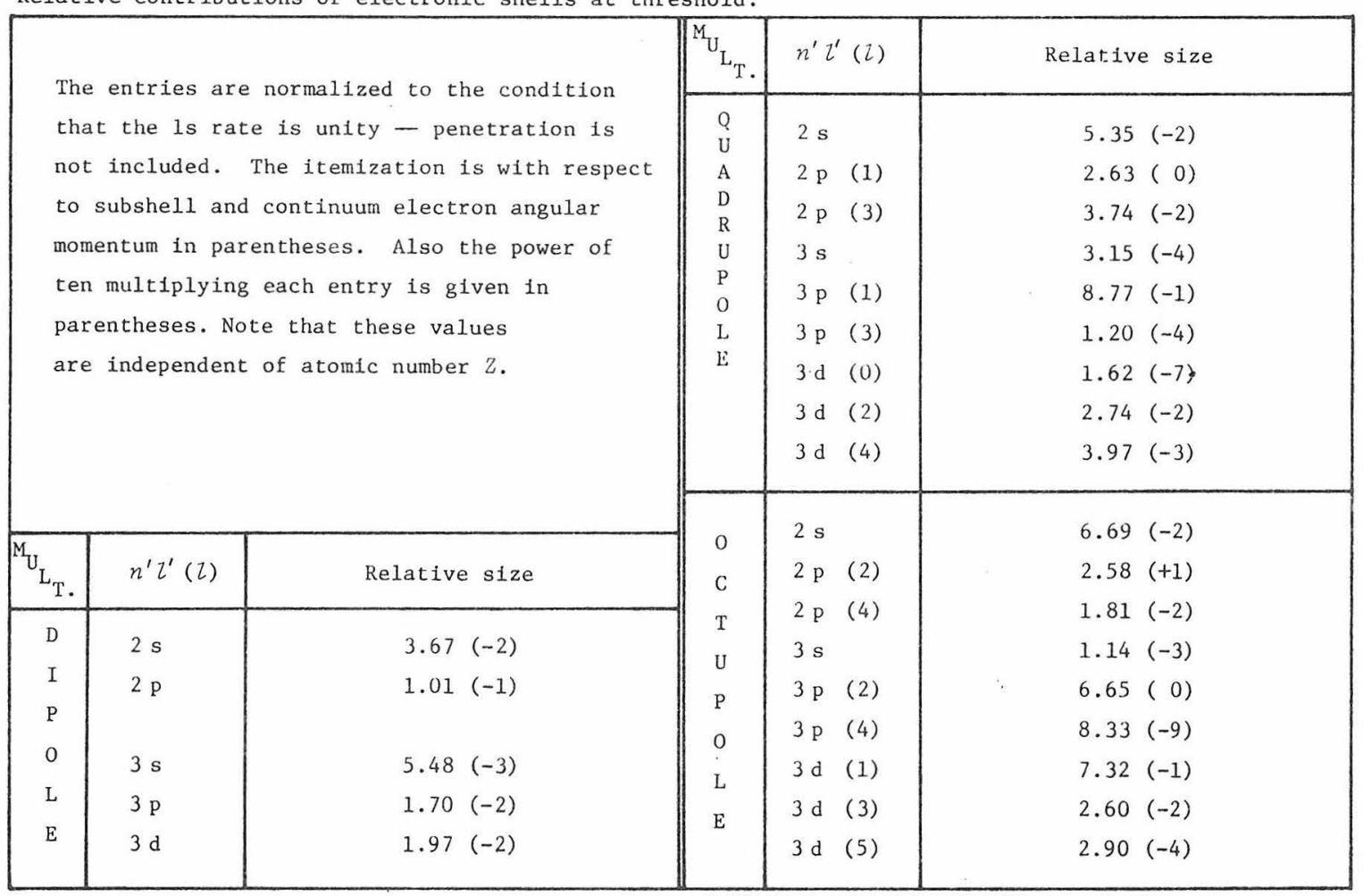


disregarded for higher multipolarities. c) With respect to electronic shells, it is mostly important for $s$ electrons (typical reduction of rates is $30 \%$ for $1 \mathrm{~s}$ and up to $100 \%$ for $2 \mathrm{~s}$ and $3 \mathrm{~s}$ electrons), has essentially no effect for the $3 d$ electrons and reduces the $2 p$ and $3 p$ rates by typically $5 \%$. $d$ ) With respect to the muonic states, the highly eccentric ones are affected more, whereas the spherical states do not penetrate much. e) The general trend, as we have mentioned in passing, is that penetration reduces rates. Nevertheless, for multipolarities $E 2$ and higher there are natural zeroes in the expressions for the rates without penetration (cf. Appendix A and ref. [51]); in cases that rates are small due to $y$ being in the vicinity of a zero, or in some other cases that cannot be readily systematized, penetration can increase the rates. Also for inherently small rates, the numerical inaccuracies of the penetration fits come in, but with inconsequential errors. To demonstrate the above points, we show in figure 2.10 some penetration graphs in the notation of ref. [52].

2.6. Further Corrections and Open Questions. Up to here we have considered effects under specific assumptions; now it is time to reconsider these approximations in the hope of estimating overall margins of safety. Hydrogen like non-relativistic wave functions, for example, are easy to work with, but are they really reliable? For some transitions there is a systematic cancellation of terms with small remainders; this precarious balance could be upset by the shifts of the peaks and nodes of the true wave functions. Such corrections, however, will influence mostly small rates and will have no effect on the overall cascade. Let us summarize some problems of intermediate difficulty.

1) Refilling of Electronic Shells. At present we handle the two ls electrons rather correctly according to ref. [53] and the rest in a crude way (refill or no refill, $i . e$, infinite or zero refilling rate respectively). A systematic algorithm for the lowest two or three shells is possible, but to implement this correctly would require a substantial expansion of the computer code. For example, several refilling rates have to be introduced $(2 p \Rightarrow 1 s, 3 p \Rightarrow 1 s, \ldots)$ and keeping track of the probabilities of all electrons is quite complicated. The refilling processes can significantly influence the cascade, especially for low $Z$ atoms.

2) Effective Charge. To correct somewhat the electronic wave functions, we have introduced an effective charge $Z^{*}$ for each shell. The best way to choose these numbers is to fit $Z^{*}$ from the Hartree-Fock wave functions (e.g. ref.[54]) in the region of small radii, where we are most sensitive. Then an average value of the subshell $Z^{*}$ could be used for the whole shell. Figure 2.11 shows such a fit and the derived effective charges.

3) Initial Distribution of the Muon. This is a wide open question, where the classical portion of the cascade could serve as a guide. In the final version of the program we have made provisions that the initial population of the muon can be spread over the entire $(n, l)$ spectrum, to account for "leakage" of muons through the starting $n$. The decision of how to spread the muon population is up to the ingenuity of the user, the agreement with the experimental data being the final judge. 
CHAPTER 3: TESTS AND COMPARISON WITH THEORY

Apart from the obvious tests of verifying the correctness of our expressions, there is a limited collection of ways to get some confidence that our approach is the correct one. Here we describe some indirect ways of checking ourselves and we leave the comparison with the experimental muonic x-ray data for next chapter, since it deserves more attention.

3.1. Theoretical Checks. As we have noted earlier, there has been a lot of pioneer work in the field of Auger transitions. Some of of the rate formulas (no penetration) can be found in the early literature $[55,56]$ and some of these results have been coded in the earlier generation cascade computer program [15]. Our results agree with those calculations to the letter, except for one very important point. In the treatment of monopole transitions ( $K$ shell, leading term of $1 / y^{2}$ expansion ( $c f$. eqns. A.21 and A.24) only, approximation for small radii) the version of the program in our possession, apart from the aforementioned approximations includes the simplification

$$
\frac{\mathrm{e}^{\pi y}}{\sinh \pi y} \sim \frac{1}{\pi y} \text {. }
$$

This unnecessary liberty, valid for $\pi y \ll 1$ is completely unacceptable where penetration, and therefore the monopole transition, is really important, i.e., for $y \geqslant 1$. In the region of interest the correct value of the expression 3.1 is approximately 2 and not a rapidly decreasing function of $y$; this means that monopole rates (the only one presently used) are underestimated by factors of as much as 10 or more, where it really counts. This irregularity has been rectified in our work.

3.2. Nuclear Internal Conversion Coefficients. The phenomenon of ejection of bound electrons to the continuum is not unique to exotic atoms. Radiation resulting from other phenomena has a chance to convert electrons to unoccupied states of the bound spectrum and more commonly to the continuum. In atomic physics one has the Auger transitions like the KLL, where a $\mathrm{K}$ shell hole is filled by an $\mathrm{L}$ electron with the simultaneous ejection of another L electron. In nuclear electromagnetic transitions a similar process takes place, that is, radiation instead of being emitted as a $\gamma$ ray gets absorbed by an atomic electron which jumps free. The main difference is that nuclear dimensions are so small that penetration is almost non existent. It turns out that in the case of no penetration the conversion coefficient (that is, the number of electrons ejected per $\gamma$ ray observed) is independent of the way that $\gamma$ ray was produced (nuclear or muonic transition or any transition for that matter). So we may compare our conversion coefficients with those of nuclear calculations; Hager and Seltzer [50] made extensive calculations using self consistent relativistic electronic wave functions. Figure 3.1 shows some typical comparison between our results and those of ref. [50]. Note that for the rates that have natural zeroes in the formulas, the nuclear calculations show a shoulder. For high electronic angular momenta and/or high multipolarities the conversion coefficients change too fast with energy and a meaningful comparison is impossible. In all these comparisons our rates are not particularly good around the threshold, where details make much more difference, like the precise position of the nodes 
Figure 3.1

Comparison of our results with nuclear internal conversion coefficients.

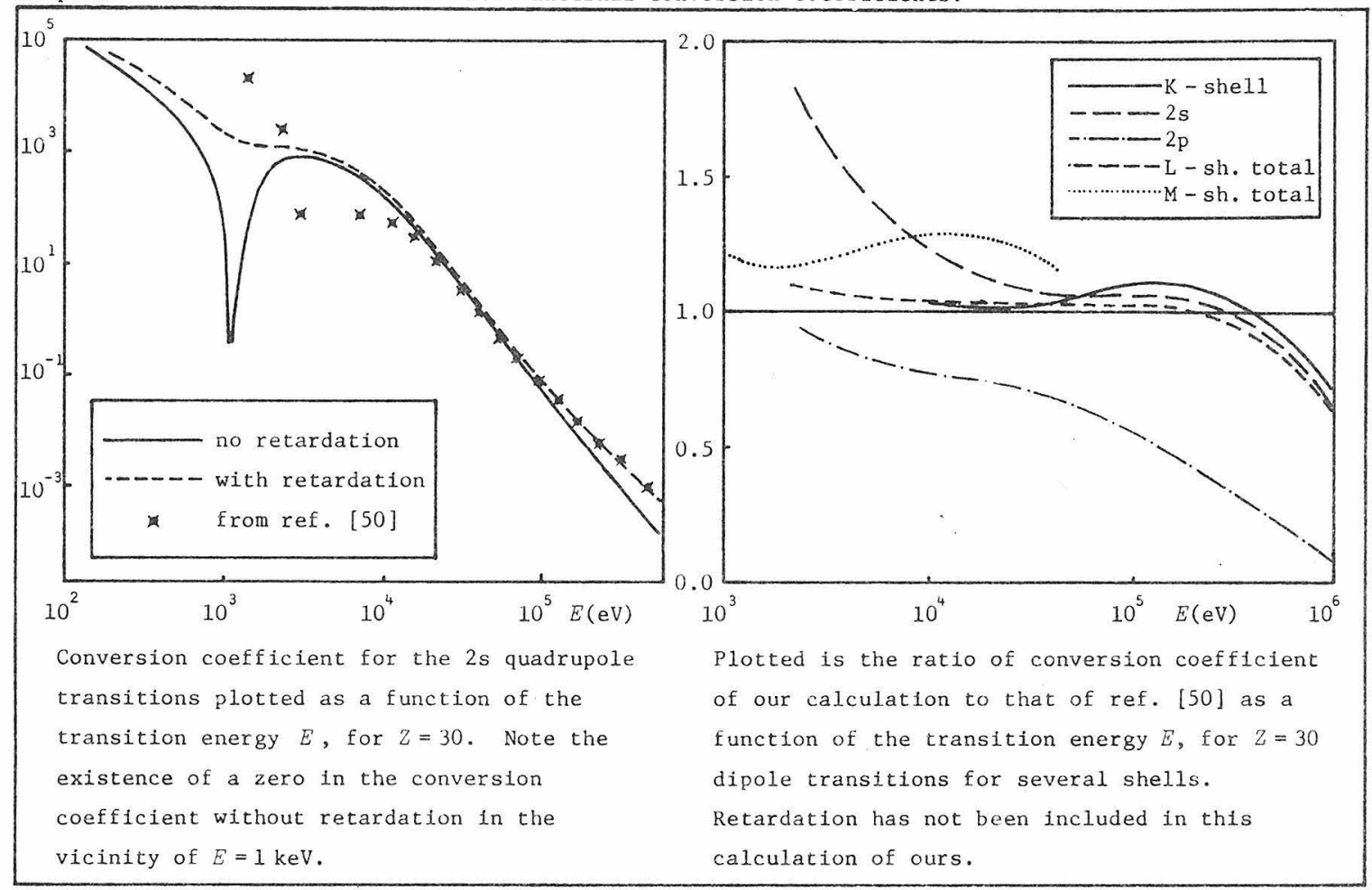

Figure 3.2

Comparison of the penetration effect with other calculations.

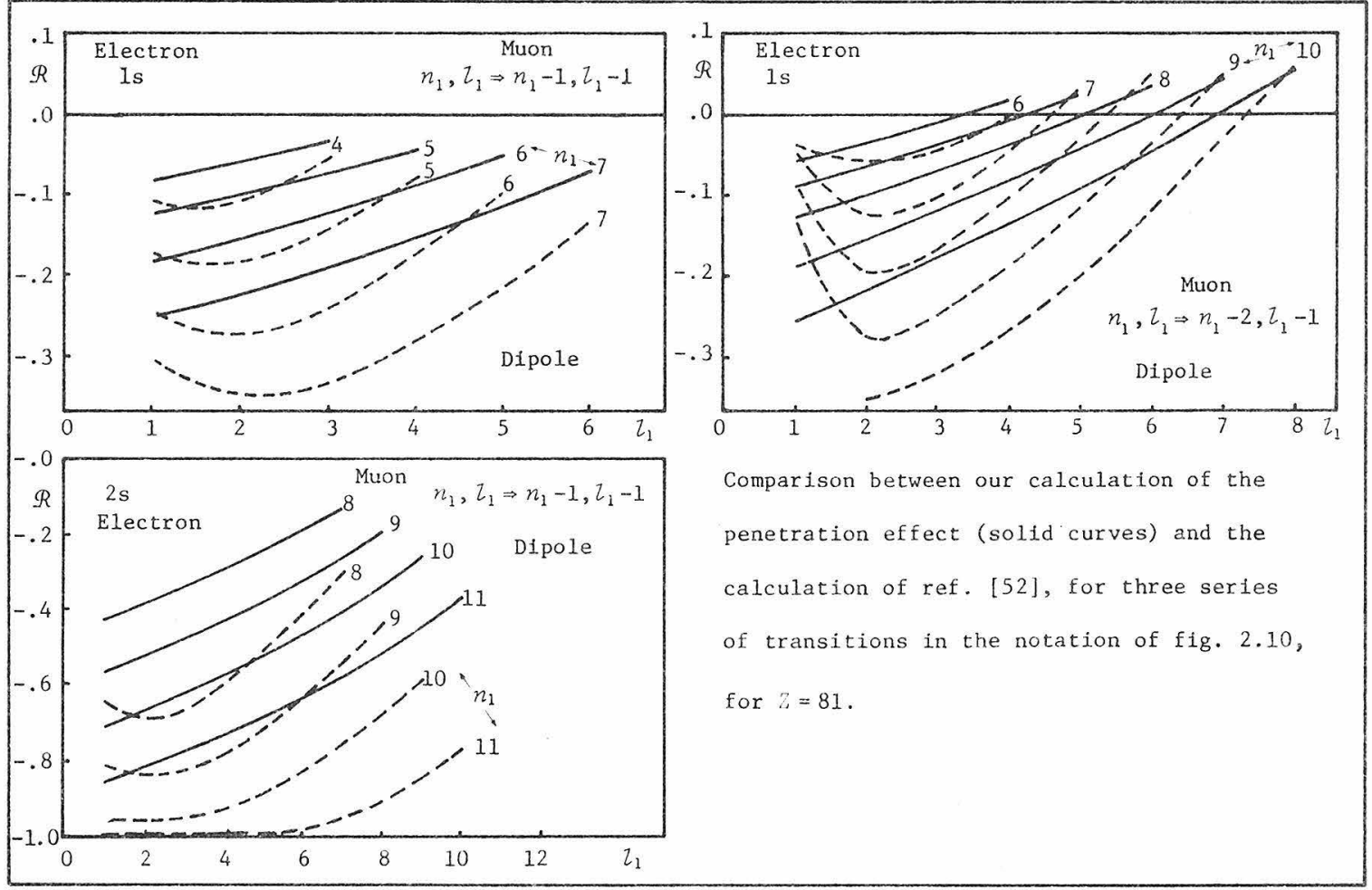


of the bound and continuum states. Nevertheless the agreement in most of the energy range is acceptable, considering the approximations involved.

3.3. Other Calculations of Penetration. Researchers in the past worried about the calculation of penetration; reference [52] is dedicated to the evaluation of penetration alone. Again, since this is a specialized piece of work, they have implemented sophisticated techniques in dealing with all the atomic subtleties. In particular for $Z=81$ the relativistic effects treated exactly in ref. [52] are quite important. We have reproduced some of the relevant figures of their paper in figure 3.2 , with our results superimposed; as one can see the two families of curves follow each other closely.

3.4. Comparison with the Photoelectric Emission of Electrons. There is a similarity between an Auger process conversion coefficient and the rate of ejection of electrons via the photoelectric effect, shown in figure 3.3. The interested reader should consult refs. [9,57] for a detailed discussion. The final result is that without too many approximations the ratio of the conversion coefficient to the photoelectric cross section is given by

$$
\frac{C(Z)}{\sigma^{\gamma}(z-1)}=\frac{1}{(Z-1)^{2}} \frac{1}{\sigma_{T}} \text {, }
$$

where $C(Z)$ is the conversion coefficient and $\sigma_{T}$ is the Thompson cross section defined by

$$
\sigma_{T}=\frac{8 \pi}{3}\left(\frac{e^{2}}{m_{e} e^{2}}\right)^{2} \doteq 0.665 \text { barns }
$$

of course, for high energies we must include retardation for the comparison to make any sense. Figure 3.4 shows such a comparison; we have plotted the combination

$$
f=\frac{C(Z)}{\sigma^{\gamma}(Z-1)}(Z-1)^{2} \sigma_{T},
$$

which ideally should be unity. The data are from reference [58], which essentially are identical to those of reference [59]. Note that in this case penetration has no meaning and that we have used dipole transitions only. The problems around thresholds still plague us as in previous tests. The effective charges $Z^{*}$ can be adjusted to get a better agreement, on the average.

The comparisons above have shown that in general our formulation is sound; problems do exist around thresholds and a perfect agreement with results that are simply more accurate cannot be expected. A method of proceeding could be to empirically discover the necessary corrections needed to minimize the discrepancies and to use them in calculating the transition rates. Rather than using such a non elegant solution, we chose to leave things as they are; we will be justified by the accomplishments of next chapter. 
Figure 3.3

Schematic representation of the photoelectric electron ejection.

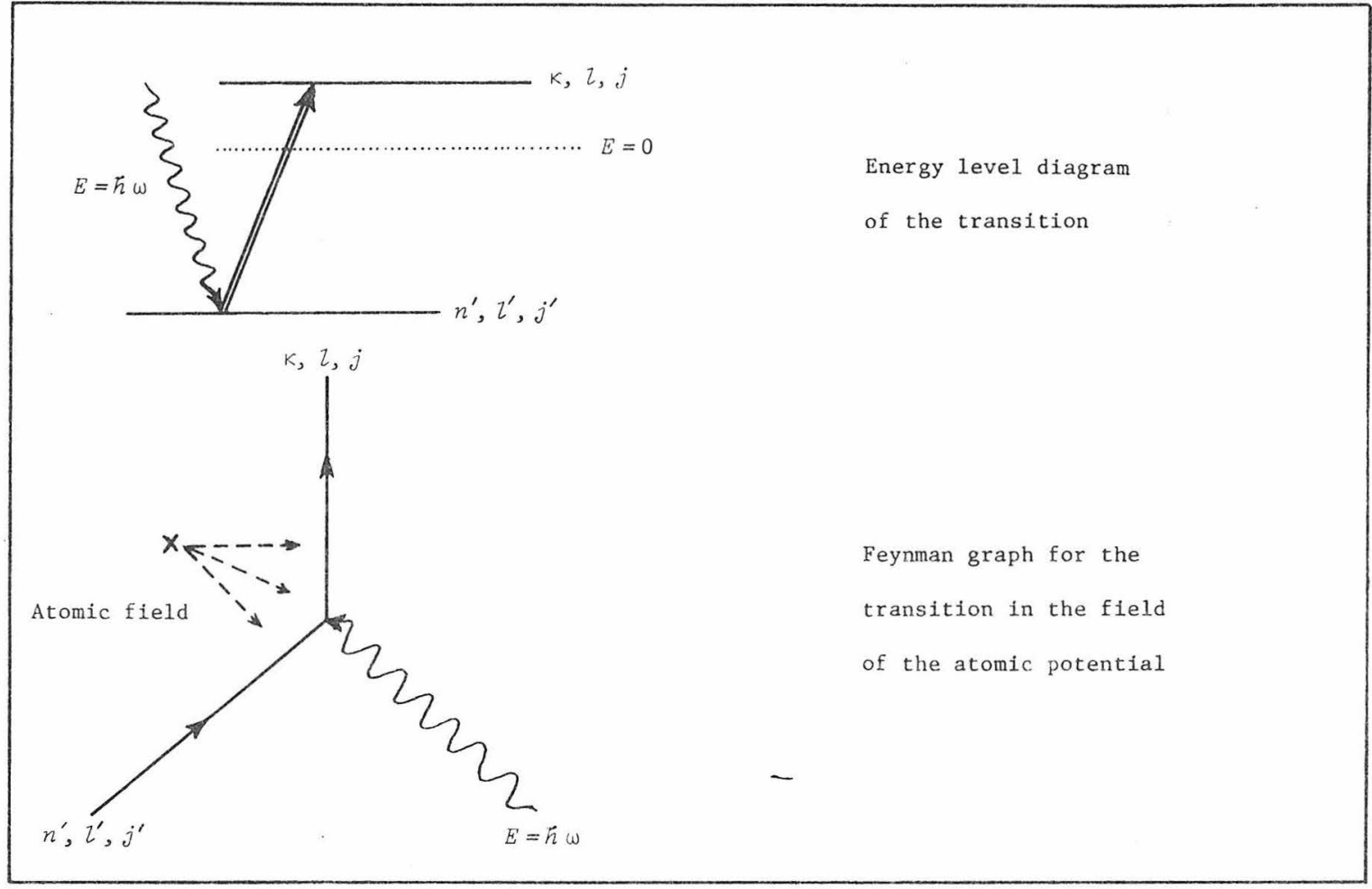

Figure 3.4

Comparison of our results with experimental photoelectric effect data.

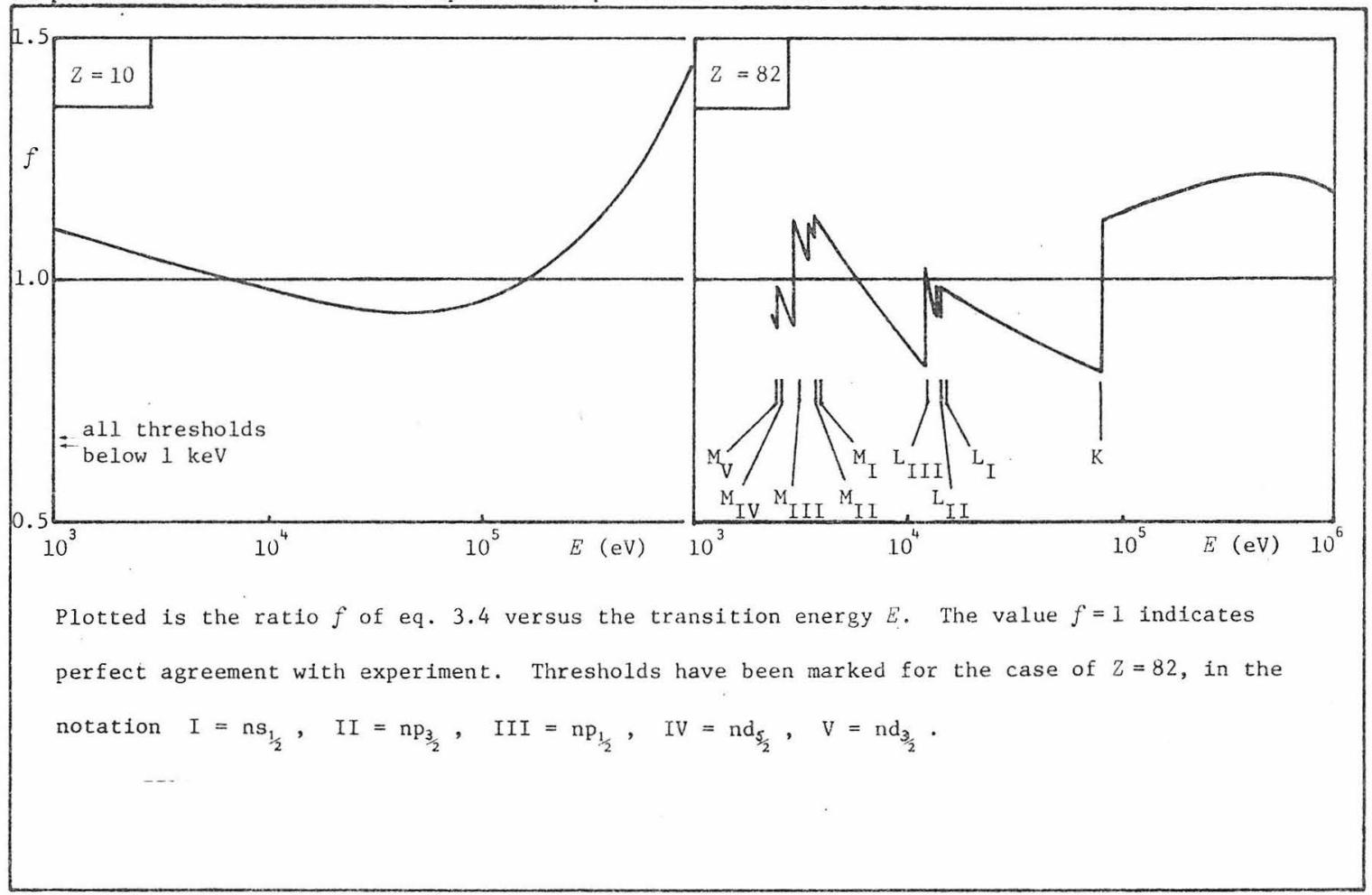


CHAPTER 4: APPLICATIONS ON THE EXPERIMENTAL RESULTS OF IRON AND THALLIUM

The acid test of our theory is to make sense of the outcome of specific muonic atom experiments. We have chosen two such experiments [48,60], which are detailed enough for our theory to give meaningful results. We shall take a closer look at them with the specific goal to understand what is happening.

4.1. General Comments on the Experiments. In view of the current state of technology, we must keep in mind that the present experiments can only measure the intensity of the muon radiative transitions above an energy threshold, over a minimum of intensity and with a specific resolution; all other information, including the Auger transitions, cannot be retrieved. Low energy transitions are naturally weak ( $e f$. the $\propto E^{2 L+1}$ basic dependence), are overwhelmed by the low energy noise and in general cannot be easily seen. It is only for low quantum numbers that individual fine structure components can be separated and only for high $Z$ atoms; in effect, the recent experimental techniques can distinguish only several tens of individual transitions and combinations thereof. Our goal is to explain the ratios found, or, if you like, we can predict these ratios for a future experiment. In trying to fit the existing data we can learn something more about the muonic atom in its early stages, by recovering some of the derived parameters. Next we examine in detail the two experiments and see hat we can learn from them.

4.2. Iron results [60]. This experiment performed by a German group at the muon channel of SIN in Switzerland and reported in 1976, has quantitatively observed 103 individual transitions or combinations in the energy range of 3 to $1900 \mathrm{keV}$ with a typical accuracy of $5 \%$ for the majority of not very weak transitions. Five series of transitions were observed ( $K, L, M, N$, and 0 , representing transitions to $n_{2}=1,2,3,4$, and 5 respectively). For the lower two series some of the fine structure components were distinguishable. To understand our problems with the quality of the fits, we must pay a closer look at the data.

4.2.1. Features of the Data. The multitude of transitions in all series make this experiment invaluable for a real test. Fractional intensities of weak lines are observed down to a few hundredths of one percent (per captured muon) with errors not more than $30 \%$ at this level. Of course, there are some difficulties in separating the close spaced weak lines, as figure 1 of reference [60] demonstrates (for example, one observes a "blob" of transitions $n_{1}$, all $l_{1} \Rightarrow 1 \mathrm{~s}$ for $n_{1} \geq 10$ ), but careful analysis has yielded values with quite tight errors on them. The other unique feature of this experiment is that there are several transitions in each of the five series. Our analysis has shown that each series is mostly sensitive to a particular region of angular momenta in the initial 2 -distribution. Thus the presence of so many, affords us the precise determination of the absolute muon population in an extended portion of the angular momentum range. In general, this is the best set of data available for this kind of experiment, and coupled to the not so high $Z$ (so that muon wave functions are reliable down to the ls level), makes it a good candidate for the determination of the reliability of our predictions. 
4.2.2. Internal Consistency Checks. It is possible to calculate the consistency or degree of "reliability" of the data, practically independent of any cascade calculation. This is done by comparing the ratio of the intensities of the transition families

$$
n p-1 s
$$

to the prediction of the theory. This way the population of the initial states cancels and since the reliability of the calculation of radiation is good (note that $2 \alpha=0.19 \ll 1$ ), we can be quite sure of the calculation. As a further means of estimating any theoretical errors due to the imperfect wave fuactions, we have performed the calculation using the uncorrected Dirac energies for all states (except the $1 \mathrm{~s}$ ) and then using the experimentally observed energies, including the effect of nuclear size, for all the low lying states. Table 4.1 compares the experimentally observed ratios (with their derived errors) with both types of predicted values. The discrepancy is indicated in the form of chi-squared per degree-of-freedom $\left(x^{2} / D F\right)$. If our predictions were perfect, this would give an unequivocal measure of the quality of the measurements and associated errors. Rather than blaming the discrepancies on the experiment, we can outright say that at least part of the disagreement is due to the imperfect calculation of the matrix elements (particularly the imperfections of the 2s wave function and the crucial position of the node for the cancellation with the highly oscillatory high-n, low- $l$ wave functions). In any case this check gives us an indication of what to expect in the comparison with the absolute rates.

4.2.3. Details of the X-Ray intensity fits. This was the first trial of our program; we decided to group together the spin multiplets (this is not really necessary, but it reduces the number of transitions to some 60 without any loss of significance). The type of parameter fit for the initial Z-distribution was decided to be the quadratic kind, since early runs of the program pointed to the fact that the population of the first few $Z$ states must be roughiy equal (see also ref. [60]). A full $(n, z)$ distribution was discarded, since it introduces an uncontrollable number of new choices. Furthermore, we had to make some choice for a few poorly determined parameters; the effective charges were taken from fittings of the wave functions around the origin (cf. fig. 2.11) and for the refilling we used values typically smaller than those found from atomic data [6l] (atomic refilling width is approximately $0.8 \mathrm{eV})$. Figure 4.1 shows the $Z$-distribution of several fits and the principal specifications under which they have been obtained. To illustrate the sensitivity of the scheme to small variations, we have plotted three similar distributions which, nevertheless give significantly higher $x^{2}$. Although the best $x^{2} / D F$ of 4.3 can not be considered satisfactory, it determines the precise shape of the 2 -distribution (assuming a model) with quite tight limits (cf. fig. 4.1). The interesting feature of all fits close to the minimal $X^{2}$ is the flatness of the distribution (although an $\tau$ independent distribution is bad $-\chi^{2} / D F=5.2$, but still far better than the unacceptable statistical distribution $\left.-\chi^{2} / D F \approx 20\right)$, predicted to a lesser extent by the fits performed by the experimenters, using the earlier cascade program. Table 4.2 shows the experimental measurements and 
TABLE 4.1

Results of the internal consistency check of the iron data.

\begin{tabular}{|c|c|c|c|c|c|c|c|}
\hline$n$ & $\mathrm{np} \Rightarrow 1 \mathrm{~s}$ & $\mathrm{np} \Rightarrow 2 \mathrm{~s}$ & Ratio $\frac{n p \Rightarrow 1 s}{n p \Rightarrow 2 s}$ & $\begin{array}{l}\text { Calculated } \\
\text { Ratio (1) }\end{array}$ & $\begin{array}{l}\chi^{2} \\
(2)\end{array}$ & $\begin{array}{l}\text { Calculated } \\
\text { Ratio (3) }\end{array}$ & $\begin{array}{c}x^{2} \\
(2)\end{array}$ \\
\hline 3 & $8.17 \pm 0.25$ & $1.21 \pm 0.10$ & $6.75 \pm 0.60$ & 5.43 & 4.84 & 7.07 & 0.28 \\
\hline 4 & $2.82 \pm 0.12$ & $0.46 \pm 0.06$ & $6.13 \pm 0.84$ & 5.26 & 1.07 & 6.34 & 0.06 \\
\hline 5 & $1.75 \pm 0.12$ & $0.36 \pm 0.04$ & $4.86 \pm 0.63$ & 5.14 & 0.20 & 6.15 & 4.19 \\
\hline 6 & $2.24 \pm 0.11$ & $0.43 \pm 0.04$ & $5.21 \pm 0.55$ & 5.16 & 0.01 & 6.04 & 2.28 \\
\hline 7 & $2.10 \pm 0.08$ & $0.34 \pm 0.04$ & $6.18 \pm 0.76$ & 5.20 & 1.66 & 5.98 & 0.07 \\
\hline 8 & $1.54 \pm 0.07$ & $0.29 \pm 0.04$ & $5.31 \pm 0.77$ & 5.21 & 0.02 & 5.93 & 0.65 \\
\hline 9 & $2.03 \pm 0.09$ & $0.42 \pm 0.03$ & $4.83 \pm 0.41$ & 5.16 & 0.65 & 5.94 & 7.33 \\
\hline 10 & $1.44 \pm 0.07$ & $0.17 \pm 0.03$ & $8.47 \pm 1.55$ & 5.14 & 4.62 & 5.91 & 2.72 \\
\hline 11 & $1.37 \pm 0.07$ & $0.33 \pm 0.03$ & $4.15 \pm 0.43$ & 5.13 & 5.19 & 5.90 & 16.56 \\
\hline 12 & $1.38 \pm 0.07$ & $--(4)$ & -- & 5.13 & -- & 5.89 & -- \\
\hline 13 & $0.58 \pm 0.06$ & $0.18 \pm 0.03$ & $3.22 \pm 0.63$ & 5.13 & 9.19 & 5.89 & 17.96 \\
\hline 14 & $0.64 \pm 0.06$ & $0.16 \pm 0.03$ & $4.00 \pm 0.84$ & 5.13 & 1.81 & 5.89 & 5.06 \\
\hline 15 & $0.40 \pm 0.06$ & $0.08 \pm 0.03$ & $5.00 \pm 2.02$ & 5.13 & 0.00 & 5.89 & 0.19 \\
\hline 16 & $0.21 \pm 0.06$ & $0.06 \pm 0.03$ & $3.00 \pm 1.55$ & 5.13 & 1.89 & 5.89 & 3.48 \\
\hline 17 & $0.21 \pm 0.06$ & $0.05 \pm 0.03$ & $4.20 \pm 2.79$ & 5.13 & 0.11 & 5.88 & 0.36 \\
\hline 18 & $0.26 \pm 0.06$ & $0.09 \pm 0.03$ & $2.89 \pm 1.17$ & 5.13 & 3.67 & 5.88 & 6.53 \\
\hline & & $x^{2}$ for & $\mathrm{s}$ & & 34.91 & & 67.72 \\
\hline
\end{tabular}

All incensities are normalized to 100 muons.

(1) Calculation performed using Schrodinger energy value for the $2 \mathrm{~s}$ muonic state.

(2) The error used in the ratio (and hence in the $x^{2}$ is calculated using the formula:

$\Delta\left(\frac{a}{b}\right)=\left\{\left(\frac{\Delta a}{b}\right)^{2}+\left(\frac{a}{b^{2}} \Delta b\right)^{2}\right\}^{\frac{1}{2}}$, where $\Delta$ stands for error.

(3) Calculation performed using experimental energy for the $2 \mathrm{~s}$ muonic state. (4) Not separable.

Figure 4.1

Fitted angular momentum distribution for iron at the beginning of the cascade

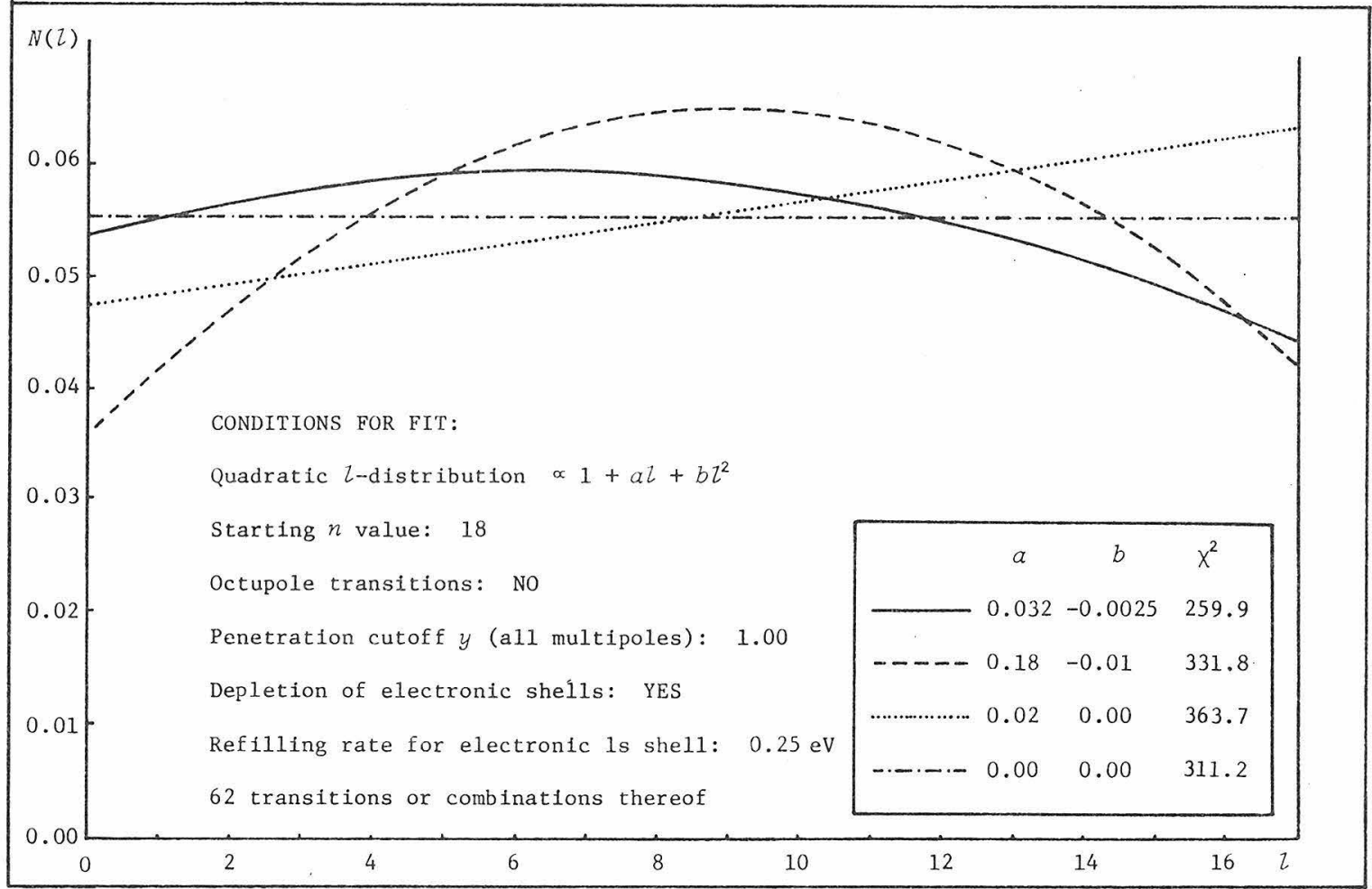


Figure 4.2

Plot of the observed and fitted $x$-ray intensities of the muonic transitions in iron.

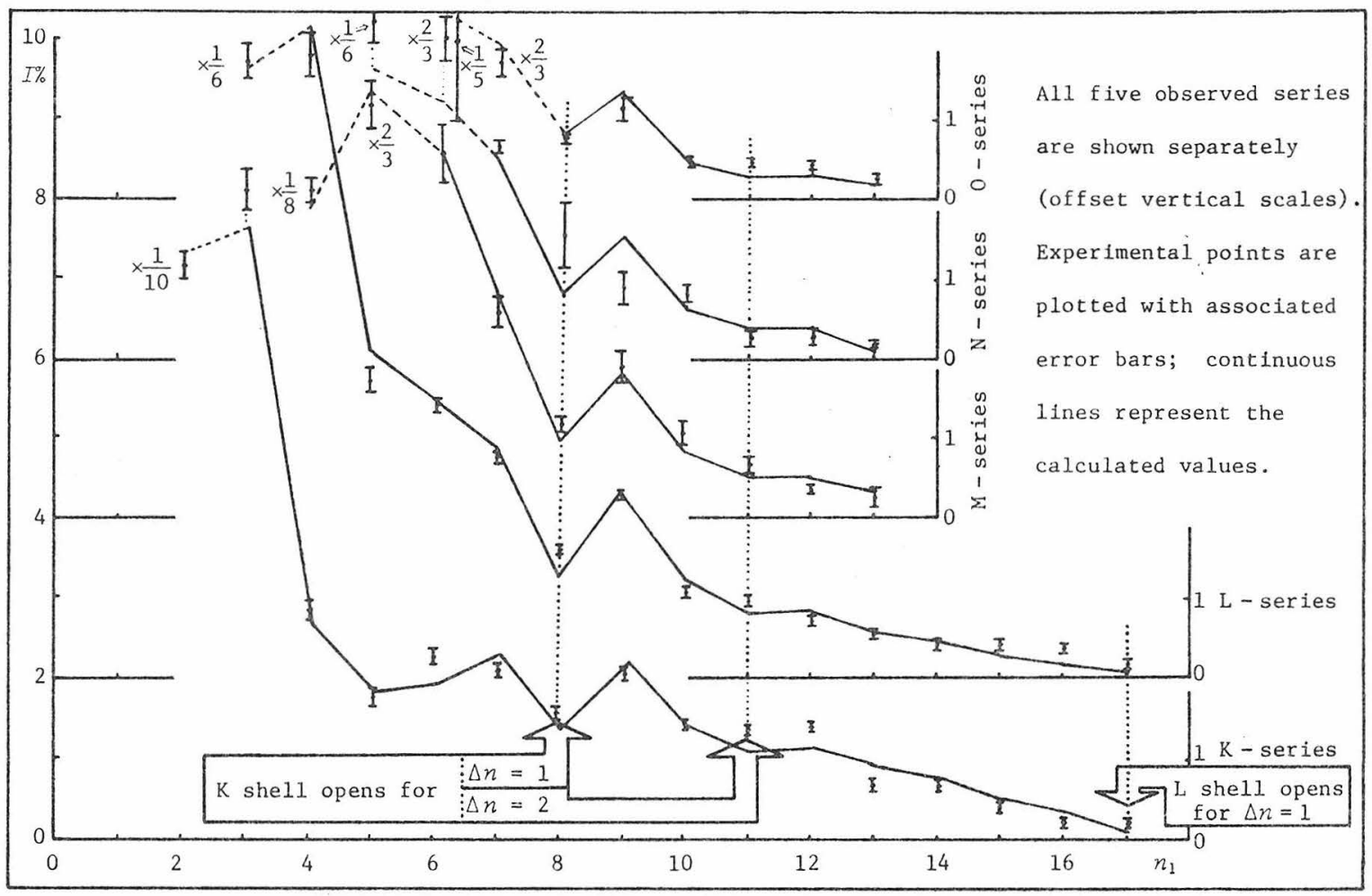

Figure 4.3

Some useful quantities of the cascade.

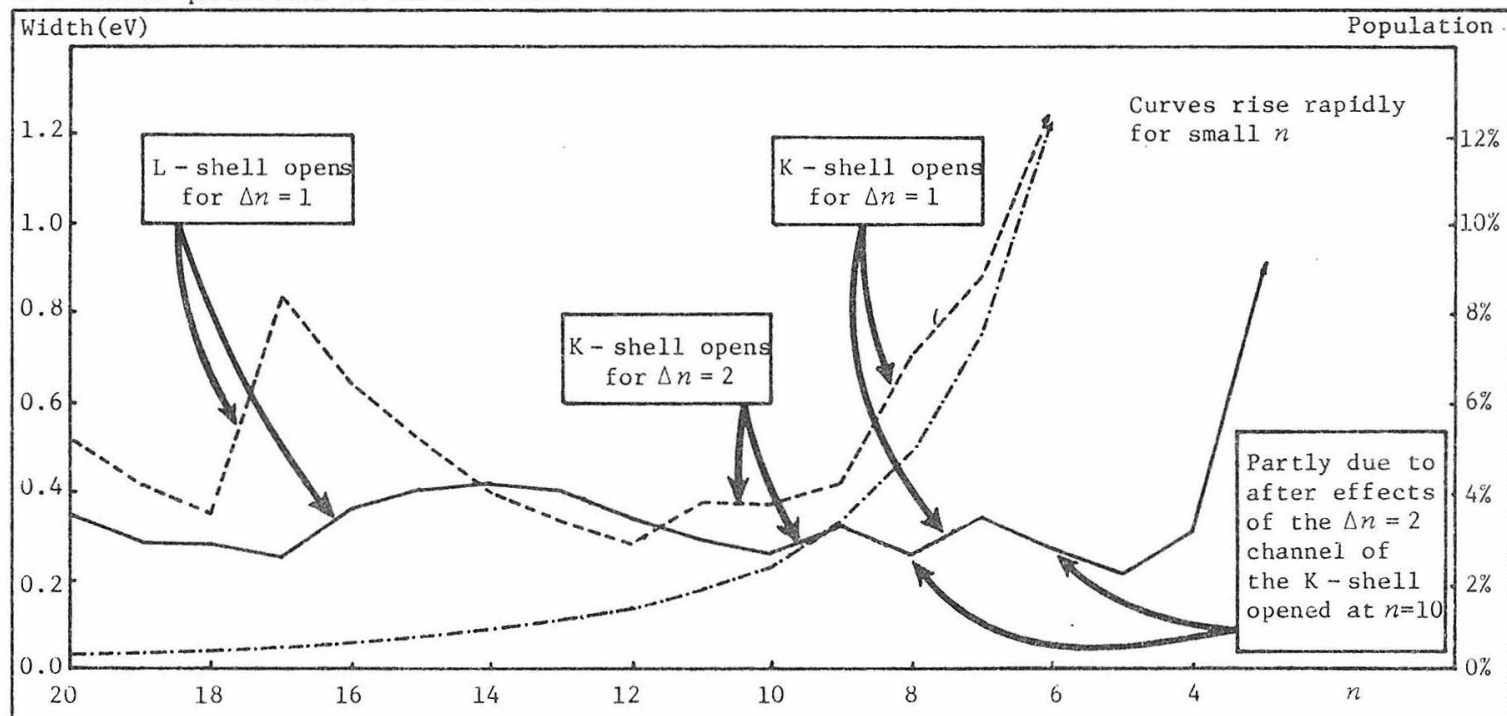

Analysis of the np states in iron. The solid curve represents the population of states with the cascade starting at $n_{1}=20$ (scale to the right). The dot-and-dash curve shows the radiative width and the dashed curve shows the total width of the states. Some of the features of these curves are noted. 
TABLE 4.2

Detailed comparison of observed and fitted $x$-ray intensities for fron.

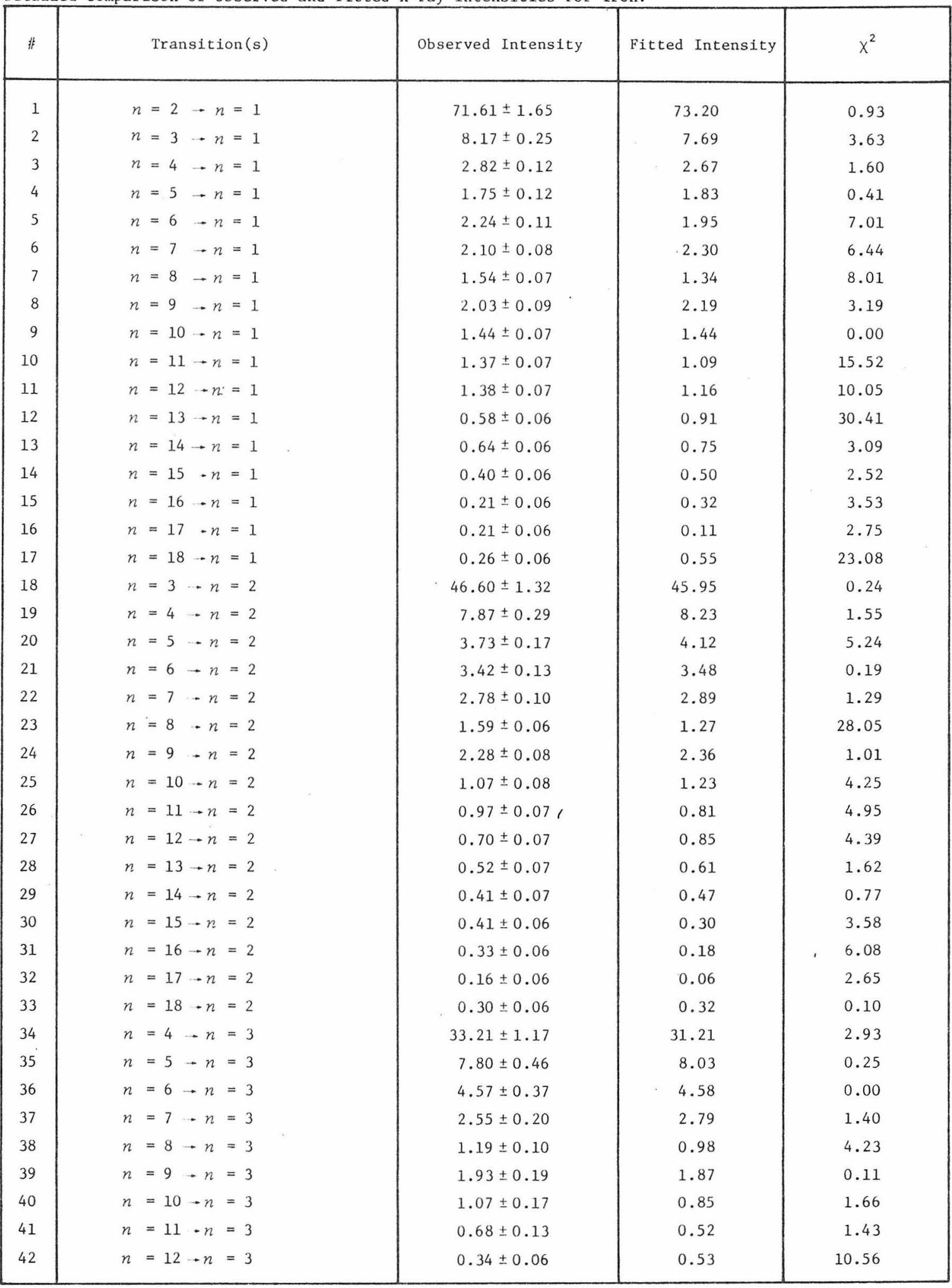

Continued next page 
TABLE 4.2 (continued)

\begin{tabular}{|c|c|c|c|c|}
\hline 非 & Transition(s) & Observed Intensity & Fitted Intensity & $x^{2}$ \\
\hline 43 & $n=13 \rightarrow n=3$ & $0.23 \pm 0.15$ & 0.37 & 0.84 \\
\hline 44 & $n=5-n=4$ & $25.66 \pm 1.75$ & 21.67 & 5.19 \\
\hline 45 & $n=6 \rightarrow n=4$ & $5.63 \pm 0.85$ & 6.13 & 0.35 \\
\hline 46 & $n=7 \rightarrow n=4$ & $2.63 \pm 0.14$ & 2.72 & 0.46 \\
\hline 47 & $n=8 \cdot n=4$ & $1.54 \pm 0.43$ & 0.81 & 2.90 \\
\hline 48 & $n=9 \quad-n=4$ & $0.90 \pm 0.22$ & 1.52 & 7.97 \\
\hline 49 & $n=10 \cdot n=4$ & $0.82 \pm 0.13$ & 0.63 & 2.13 \\
\hline 50 & $n=11 \rightarrow n=4$ & $0.24 \pm 0.10$ & 0.37 & 1.76 \\
\hline 51 & $n=12 \rightarrow n=4$ & $0.26 \pm 0.10$ & 0.37 & 1.28 \\
\hline 52 & $n=13 \rightarrow n=4$ & $0.15 \pm 0.03$ & 0.25 & 10.85 \\
\hline 53 & $n=6 \rightarrow n=5$ & $9.90 \pm 5.00$ & 11.56 & 0.11 \\
\hline 54 & $n=7 \rightarrow n=5$ & $2.62 \pm 0.28$ & 2.96 & 1.46 \\
\hline 55 & $n=8-n=5$ & $0.73 \pm 0.06$ & 0.72 & 0.03 \\
\hline 56 & $n=9-n=5$ & $1.14 \pm 0.16$ & 1.32 & 1.27 \\
\hline 57 & $n=10 \rightarrow n=5$ & $0.48 \pm 0.06$ & 0.50 & 0.14 \\
\hline 58 & $n=11-n=5$ & $0.45 \pm 0.06$ & 0.29 & 7.39 \\
\hline 59 & $n=12-n=5$ & $0.41 \pm 0.06$ & 0.28 & 4.61 \\
\hline 60 & $n=13-n=5$ & $0.24 \pm 0.08$ & 0.18 & 0.51 \\
\hline \multicolumn{4}{|c|}{ Total $x^{2}$ for 60 transitions fitted: } & 259.94 \\
\hline
\end{tabular}

Figure 4.4

Fitted angular momentum distribution for thallium at the beginning of the cascade.

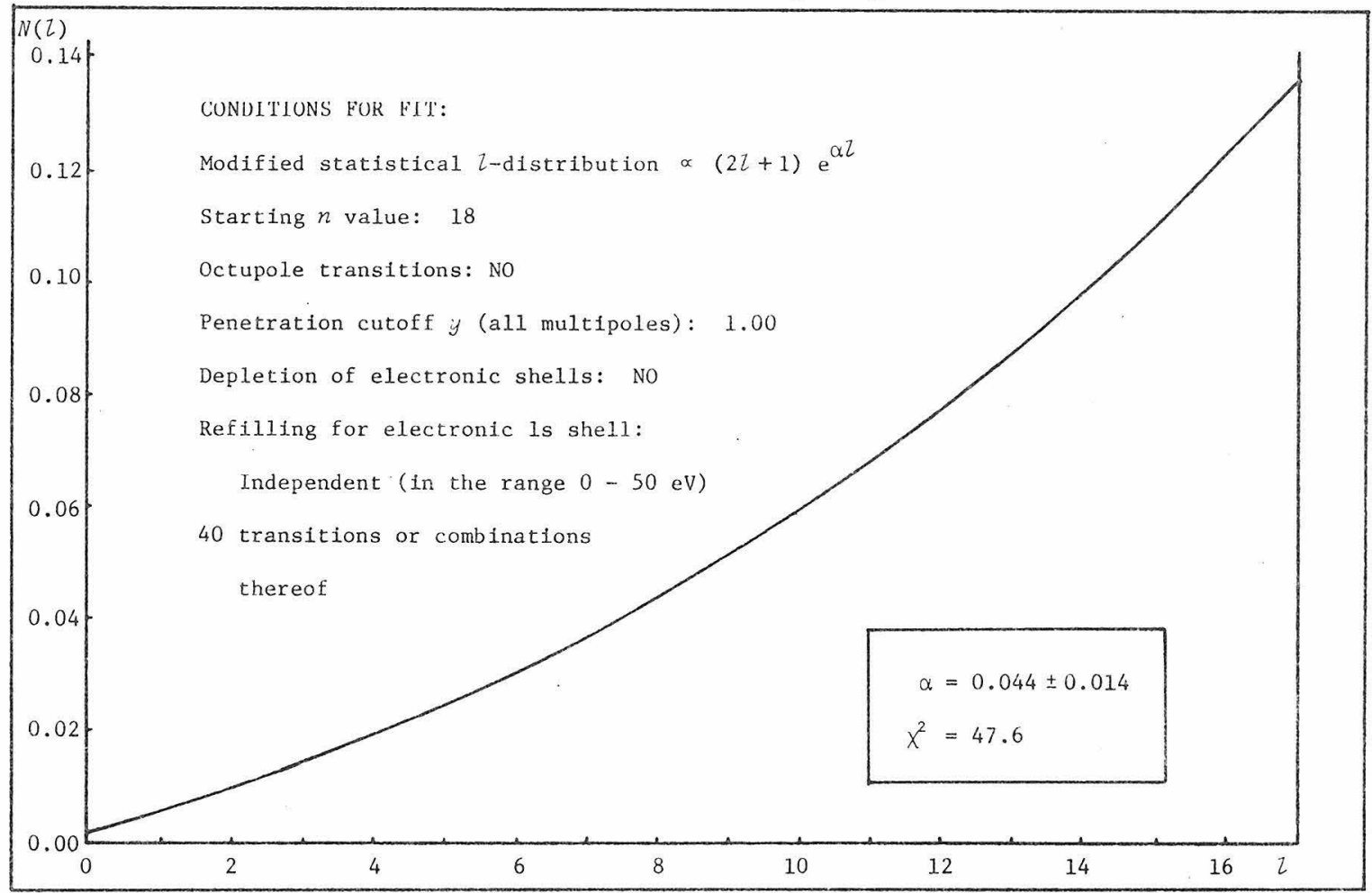


TABLE 4.3

Detailed comparison of observed and fitted x-ray intensities for thallium.

\begin{tabular}{|c|c|c|c|c|}
\hline$\#$ & Transition(s) & $\begin{array}{l}\text { Observed } \\
\text { Intensity }\end{array}$ & $\begin{array}{l}\text { Fitted } \\
\text { Intensity }\end{array}$ & $x^{2}$ \\
\hline 1 & $n=7 \rightarrow n=6$ and $n=11 \rightarrow n=8$ & $45.50 \pm 7.00$ & 50.93 & 0.53 \\
\hline 2 & $n=9 \cdot n=7$ & $8.00 \pm 1.20$ & 8.66 & 0.31 \\
\hline 3 & $n=12 \rightarrow n=8$ & $1.30 \pm 0.20$ & 1.59 & 2.06 \\
\hline 4 & $n=13-n=8$ & $0.95 \pm 0.15$ & 0.87 & 0.31 \\
\hline 5 & $n=10 \cdot n=7$ & $3.70 \pm 0.30$ & 3.27 & 2.04 \\
\hline 6 & $n=8-n=6$ and $n=11 \rightarrow n=7$ & $9.00 \pm 1.40$ & 9.65 & 0.21 \\
\hline 7 & $n=6-n=5$ & $60.00 \pm 8.00$ & 58.04 & 0.06 \\
\hline 8 & $n=12-n=7$ & $0.90 \pm 0.15$ & 1.02 & 0.66 \\
\hline 9 & $n=13 \rightarrow n=7$ & $0.70 \pm 0.09$ & 0.63 & 0.59 \\
\hline 10 & $n=9-n=6$ & $3.15 \pm 0.34$ & 3.17 & 0.01 \\
\hline 11 & $n=10 \rightarrow n=6$ & $1.90 \pm 0.30$ & 1.56 & 1.27 \\
\hline 12 & $n=11 \rightarrow n=6$ & $1.00 \pm 0.10$ & 0.99 & 0.01 \\
\hline 13 & $n=7 \rightarrow n=5$ & $10.40 \pm 0.90$ & 9.31 & 1.47 \\
\hline 14 & $n=12-n=6$ & $0.57 \pm 0.16$ & 0.66 & 0.33 \\
\hline 15 & $5 g_{7 / 2}-4 f_{7 / 2}$ & $1.62 \pm 0.26$ & 1.02 & 5.37 \\
\hline 16 & $5 g_{\frac{9}{2}}^{2}-4 f_{\frac{7}{2}}^{2}$ & $36.60 \pm 5.70$ & 35.62 & 0.03 \\
\hline 17 & $5 g_{\frac{7}{2}}-4 f_{\frac{5}{2}}, 5 f_{\frac{7}{2}}-4 d_{\frac{5}{2}}$, and $5 f_{\frac{5}{2}}-4 d_{\frac{5}{2}}$ & $30.20 \pm 4.50$ & 39.54 & 0.02 \\
\hline 18 & $5 \mathrm{f}_{\frac{5}{2}} \rightarrow 4 \mathrm{~d}_{\frac{7}{2}}$ & $1.17 \pm 0.26$ & 1.38 & 0.65 \\
\hline 19 & $n=8 \cdot n=5$ & $2.80 \pm 0.30$ & 2.63 & 0.32 \\
\hline 20 & $n=9 \rightarrow n=5$ & $2.60 \pm 0.70$ & 1.31 & 3.38 \\
\hline 21 & $9 f_{\frac{7}{2}}-5 d_{5 / 2}$ and $9 g_{7 / 2}-5 f_{\frac{5}{2}}$ & $1.50 \pm 0.50$ & 0.18 & 6.98 \\
\hline 22 & $6 \mathrm{~g}_{\frac{9}{2}} \rightarrow 4 \mathrm{f}_{7 / 2}$ & $3.90 \pm 0.80$ & 4.00 & 0.02 \\
\hline 23 & $6 \mathrm{~g}_{\frac{7}{2}} \rightarrow 4 \mathrm{f}_{\frac{5}{2}}, 6 \mathrm{f}_{\frac{7}{2}} \rightarrow 4 \mathrm{~d}_{\frac{5}{2}}$, and $6 \mathrm{f}_{\frac{5}{2}} \rightarrow 4 \mathrm{~d}_{\frac{7}{2}}$ & $3.80 \pm 0.80$ & 3.91 & 0.02 \\
\hline 24 & $7 g_{g / 2}-4 f_{\frac{7}{2}}$ and $\quad 7 g_{\frac{g}{2}} \quad 4 f_{\frac{7}{2}}$ & $1.20 \pm 0.30$ & 1.09 & 0.13 \\
\hline 25 & $7 g_{7 / 2}-4 f_{5 / 2}$ & $1.30 \pm 0.30$ & 0.82 & 2.58 \\
\hline 26 & $4 d_{5} / 2 \rightarrow 3 p_{3 / 2}$ & $1.00 \pm 0.30$ & 0.87 & 0.18 \\
\hline 27 & $4 \mathrm{~d}_{3 / 2}-3 \mathrm{p}_{\frac{1}{2}}, 4 \mathrm{~d}_{\frac{3}{2}}-3 \mathrm{p}_{\frac{3}{2}}$, and $4 \mathrm{f}_{\frac{5}{2}}+3 \mathrm{~d}_{5 / 2}$ & $2.50 \pm 0.50$ & 2.72 & 0.19 \\
\hline 28 & $4 \mathrm{f}_{7},-3 \mathrm{~d}_{5} / 2$ & $38.70 \pm 7.80$ & 42.71 & 0.26 \\
\hline 29 & $4 \mathrm{f}_{5 / 2} \rightarrow 3 \mathrm{~d}_{3 / 2}$ & $29.20 \pm 6.00$ & 29.90 & 0.01 \\
\hline 30 & $2 \mathrm{~s} \frac{\mathrm{l}}{2}-2 \mathrm{p}_{\frac{1}{2}}$ & $0.55 \pm 0.22$ & 0.78 & 1.13 \\
\hline 31 & $5 g \frac{9}{2}-3 d_{5 / 2}$ (quadrupole) & $0.80 \pm 0.20$ & 0.64 & 0.66 \\
\hline 32 & $5 \mathrm{f}_{7 / 2} \rightarrow 3 \mathrm{~d}_{5 / 2}$ and $5 \mathrm{f}_{\frac{5}{2}} \rightarrow 3 \mathrm{~d}_{\frac{5}{2}}$ & $3.80 \pm 0.80$ & 3.64 & 0.04 \\
\hline 33 & $5 f_{\frac{5}{2}}-3 d_{\frac{3}{2}}$ & $2.10 \pm 0.40$ & 2.42 & 0.66 \\
\hline 34 & $5 g_{\frac{7}{2}} \rightarrow 3 d_{3 / 2}$ (quadrupole) & $0.40 \pm 0.10$ & 0.46 & 0.35 \\
\hline 35 & $3 p_{\frac{1}{2}}-2 s \frac{1}{2}$ & $0.71 \pm 0.18$ & 0.44 & 2.27 \\
\hline 36 & $3 p_{\frac{3}{2}}-2 s \frac{1}{2}$ & $0.54 \pm 0.19$ & 0.88 & 3.17 \\
\hline 37 & $4 d_{\frac{3}{2}} \rightarrow 2 p_{3} / 2$ & $0.30 \pm 0.20$ & 0.28 & 0.01 \\
\hline 38 & $4 \mathrm{f}_{\frac{5}{2}} \rightarrow 2 \mathrm{p}_{3 / 2}\left(\right.$ quadrupole) and $4 \mathrm{~d}_{5 / 2}+2 \mathrm{p}_{3 / 2}$ & $1.50 \pm 0.60$ & 2.77 & 4.45 \\
\hline 39 & $4 \mathrm{f}_{7} / 2-2 \mathrm{p}_{3} / 2$ (quadrupole) & $1.10 \pm 0.30$ & 1.26 & 0.27 \\
\hline 40 & $4 \mathrm{f}_{\frac{5}{2}}-2 \mathrm{p}_{1 / 2} \quad$ (quadrupole) & $0.30 \pm 0.20$ & 0.73 & 4.67 \\
\hline & Total $x^{2}$ for $40 \mathrm{tr}$ & & & 47.66 \\
\hline
\end{tabular}


errors together with our predictions and some other useful information; note that a substantial contribution to the total $X^{2}$ comes from a small number of badly predicted lines. Figure 4.2 shows the same transitions (fine structure components added) plotted as a function of the initial $n_{1}$ for each series. Although we are two standard deviations off the measured values on the average, we correctly reproduce all qualitative features of the data. The three arrows point to the quantum numbers where major Auger transition channels open; the result of this is that the total width of these states is increased due to the additional Auger rates, and therefore radiation decreases in proportion. This phenomenon accounts for most of the highs and lows of the intensities within each series. Another inceresting feature is the effect of the refilling rate of the $K$ shell electrons. It primarily affects the intensity of the transitions originating from the $n_{1}=7$ level for the following reason. The $\mathrm{K}$ shell opens for $\Delta n=1$ at $n_{1}=8$ and till then the population of the $K$ shell is almost as much as the initial one; now the $8 \Rightarrow 7$ Auger transition is so strong, that just about one full electron is ejected. If there is no refilling, the Auger rate from the $n_{1}=7$ level is half of what it would have been with infinitely fast refilling, and the relative role of the radiation (which is typically as strong as the Auger rates at that level) is changed accordingly. Similar effects happen to a lesser extent at around $n_{1}=17$ for the $L$ shell. So in effect we have two ways of changing the population of the muonic states, one by specifying a different number in the beginning which means that a different number will trickle through via the cascade. On the other hand one may change the refilling rate, which affects only a few selected regions of states. Other parameters affect the branching ratios to a lesser extent (if those parameters are kept within rational limits).

Another informative set of quantities is shown in figure 4.3. As the different Auger channels open, the population of the respectively affected muonic states increases; this increase is reflected in the decrease of the radiation width as explained above and in the case of $\Delta n=2$ for $K$ shell it carries over to the lower states modulo 2, viz., this decrease of the radiative width occurs every other $n_{1}$. Finally at the very low quantum numbers radiation becomes most important and the Z-distribution becomes quite steep.

Judging from the successful results above we can say that the fact that we cannot get a $x^{2} / D F$ in the neighborhood of unity probably should not be ascribed to the data. Rather it appears that we cannot fit perfectly data that are typically $5 \%$ accurate with a theory that is not that accurate. Note that the inaccuracies of the theory have not been included in the calculation of the $x^{2}$, since they are not randomly distributed and they can not be firmly estimated. Nevertheless, this does not preclude the use of the program to fit experiments with similar features, since sensible fits can be derived and parameters can be determined with reasonable degree of certainty. This will become apparent in the analysis of the next experiment.

4.3. Thallium Results [48]. This is an older experiment (1971) by a German-Swiss group at the muon channel at CERN. The energy interval scanned ranged fron 130 to $3500 \mathrm{keV}$. Only $40 \mathrm{sets}$ of transitions were observed (none in the $\mathrm{K}$ series which starts at higher energies). 
4.3.1. Features of the Data. In this case there are few transitions with low intensities and the typical accuracy is $10 \%$ for the majority of lines. A large number of these transitions are between states of high $n_{1}$ and $n_{2}$ (in these cases the fine structure components overlap and cannot be separated). Since there is no $K$ series in the data (the $K$ series is hard to analyze due to the hyperfine splitting and has been reported elsewhere) and the $\mathrm{L}$ series is short, the $Z$-distribution cannot be tested as closely as in the case of iron. In addition the high 2 makes Auger rates less important and that increases the reliability of the calculation. Finally, there is no possibility of the internal consistency check of section 4.2.2.

4.3.2. Details of the X-Ray Intensity Fits. The modified statistical distribution $\left(\propto(2 Z+1) e^{\alpha} \tau\right.$ ) seemed appropriate and adequate for this case. It turns out that for thallium the initial 2 -distribution is quite steeper than statistical, as shown in figure 4.4. The effective charges were derived as before and a separate fit was run for them; the $X^{2} / D F$ does not depend much on the effective charges and in this case the refilling rate for the $\mathrm{K}$ shell is almost irrelevant if kept smaller than the atomic value of $50 \mathrm{eV}$. The rest of the conditions together with the actual numbers are in table 4.3, alongside the data and errors. The $x^{2} / D F$ is about 1.2 , which means that we can fit the results with reasonable accuracy and the reliability of the calculation is comparable to (or better than) the typical experimental accuracies. Note that the fit is slightly better when fast refilling is assumed for the L-shell.

4.4. Other experiments. Muonic transitions have been observed for several years. Many other experiments exist (vide, for example, [62]), but the problem is that only a limited number of primarily low quantum number transitions have been recorded. Under these circumstances, reliable fits of the initial l-distribution and extrapolations to high levels are just about impossible. Future experiments with many precise transitions will provide tests for the theory, used either as a prediction guide or an analysis tool. Another side use of the program is the calculation of the polarization; there are several experimental results ( $f f$. sect. 1.4) which can be checked.

4.5. Conclusions on the Angular Momentum Distribution. As we have seen a distribution close to statistical, as predicted naively from the slow down processes or as calculated more accurately (fig. 1.7), is not compatible with the data in either case, especially for iron, whose initial l-distribution is almost "flat", i.e., independent of $Z$. Thallium is about three standard deviations off an exact statistical distribution. The present theory is not adequate in accomnodating the experimental observations and it is left as a challenge for future work on the capture mechanisms. More experiments of the quality of iron [60] would be desirable in order to facilitate the development of the theory. 
CHAPTER 5: DESCRIPTION OF THE CASCADE PROGRAM

To make the results of our work usable, we have written a general purpose computer program, which incorporates several newly established findings. This program is an upgraded version of the program CASCADE [15] and a manuscript, consisting of a brief out line of the theory and a detailed description of the input and output options, has been accepted for publication in the Computer Physics Communications Journal [63]. A computer readable source and a sample test run is available. This chapter gives an overview of the structure of the program, without going into the technical details; for a complete description, consult the writeup [64]. In figure 5.1 we have reproduced the hierarchy of the subroutines used, to give a schematic indication of the level of complexity involved.

5.1. General Features. The most important consideration for such a program is speed versus accuracy, for a program that requires too much time is not very practical, and conversely, if it is not sufficiently accurate, it is not of much use, anyway. Having this in mind, we incorporated most of the new results of the previous chapters; on the other hand, disregarded other possible corrections in favor of practical considerations (computing speed and programming simplicity). Following is a recapitulation of the basic approximations made in the program.

1) The wave functions for both the electrons and the muon have been taken as the simplest possible, viz: nonrelativistic hydrogen like. As a means of correcting the electronic wave functions,

Figure 5.1

Hierarchical arrangement of the subroutines in the program.

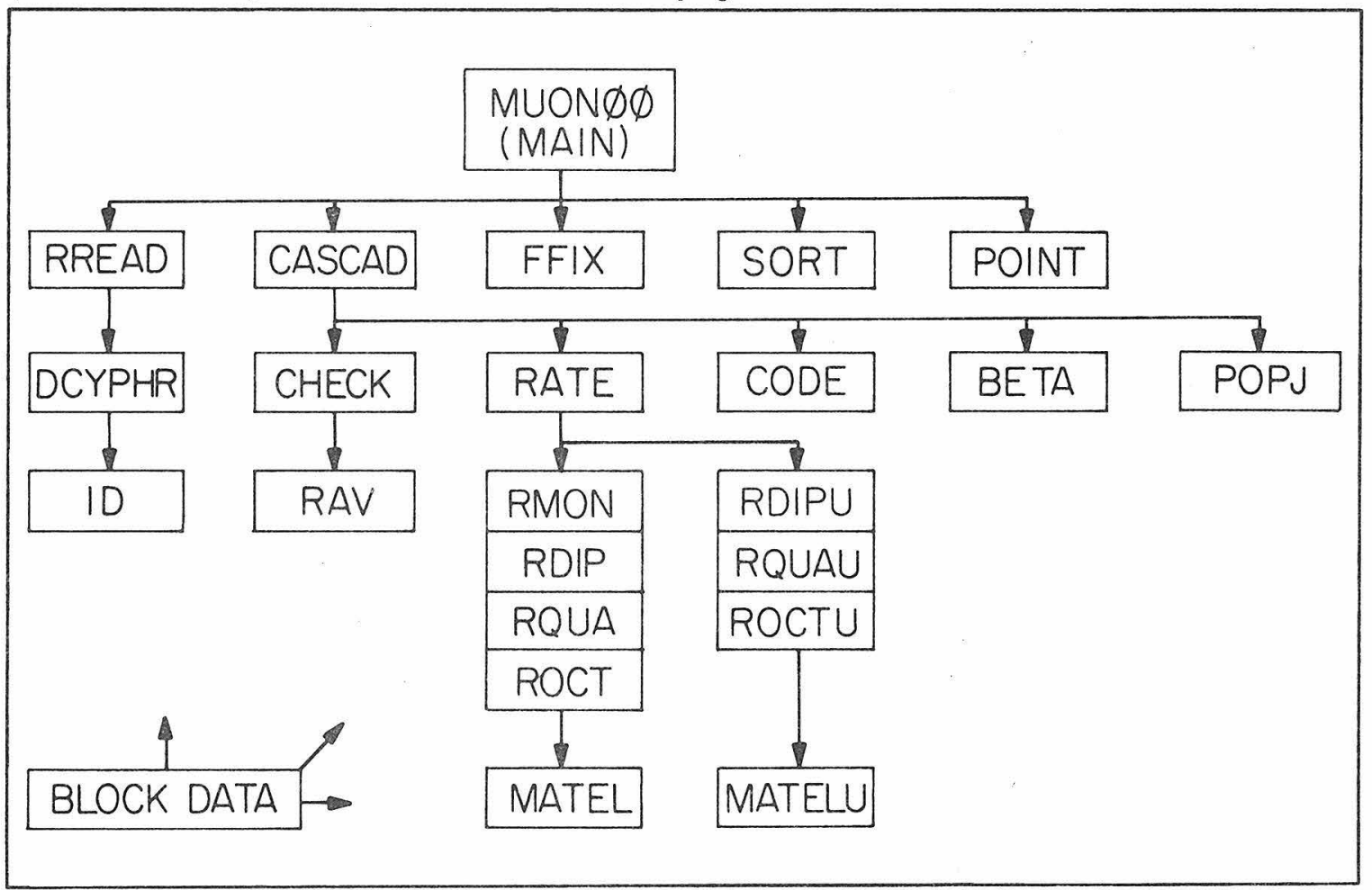


an effective charge $Z^{*}$ is adopted for each shell, to simulate the effect of the distortion of the atomic potential. A more exact calculation would involve Hartree-Fock wave functions, which to a high degree of accuracy could be written as linear combinations of products of powers and exponentials of the radial coordinate (radial part) [28] and, therefore, the integrals of the matrix elements could be performed in the same way as in the case of hydrogen like wave functions. When we consider the continuum electron states, the problem becomes quite complicated, if we want to have an accurate description (especially near the threshold), since there is a continuous dependence on the momentum of the electron. The present calculation is reliable away from the thresholds, but to have a very exact pieture, it would require great sacrifice of computational speed. Considering the muon, the induced error is small, since the high lying states are quite hydrogen like, whereas for the low lying states the radiation rates are so overwhelming, and considering the strong energy dependence of these rates ( $\propto E^{2 L+1}$ ), the branching ratios of the decay schemes are not much influenced by the detailed form of these wave functions.

2) Multipolarities up to octupole and electronic shells up to $M$ are considered. For the range of muon energies that the program is intended $\left(n_{1} \leq 20\right)$, these limitations are adequate, since the $N$ and higher shell thresholds correspond to much higher $n_{1}$ values, and we have seen that the octupole transitions already do not contribute significantly to the rates. Once more, near thresholds (in the case of the $\mathrm{L}$ shell threshold for $\Delta n=1$, for example) higher multipoles could be significant. An important remark is that the inclusion of $E 2$ and to lesser extent $E 3$ transitions changes the rates to such an extent that, coupled with the larger $\Delta l$ involved, the fits for an initial $Z$-distribution are substantially different.

3) The penetration as explained in Appendix A is treated approximately, but in a reliable way.

4) Auger transitions to bound electronic states have been ignored.

5) As we have shown, the relativistic retardation and electronic screening effects, although simple enough to calculate, do not affect the rates in an appreciable manner and, therefore, they have not been programmed.

6) The population of the components of the muon fine structure doublets is treated statistically, which is sound as long as the radial parts of the wave functions are the same. In practice this is quite adequate and it is corroborated by experimental findings $[60\}$.

7) The problem of electron refilling (the rate with which an atomic level is refilled after the electron has been ejected via an Auger transition) is treated correctly for the $\mathrm{K}$ shell using an externally controlled refilling rate [53], but it is rather crudely done for the higher shells, being taken either as instant (infinite rate) or none (zero rate) according to the choice of the user.

8) In the latest version of the program we have made provisions for the user to assign initial populations to the full $(n, l)$ spectrum of muonic states. This extention of the original $l$-distribution at the starting $n_{1}$, should fulfill any possible needs to that effect.

Other phenomena disregarded are indeed negligible, such as electron-positron pair production by 
the muon, or higher order perturbation expansion terms. Since the program is expected to be run by a diverse group of users, standard FORTRAN IV features were implemented.

5.2. Input and Output. The primary object of the program is to compute the $x$-ray intensities of the muonic transitions and thereby allow the user to fit for some undetermined parameters by comparing the theoretical predictions with the experimentally observed intensities; conversely, given the required input parameters, one can predict what an experimental run should yield, and thereby use the results as a pre-experiment guideline. In any case, the user must be able to specify what he wants the program to do and to be able to retrieve the results. In the following section we will discuss more closely the kinds of specifications the user can input. The output includes a summary of quantities of interest resulting from the cascade and a catalogue of $x$-ray intensities arranged in order of increasing transition energy. The user can punch any transitions that would be of particular interest, for further processing.

5.3. Selection of Input Options. The format of the input has been simplified and the user can override the internally set default parameters, if it is so desired, to adapt the program to the particular case. The interested reader should consult ref. [64] for a comprehensive explanation of all options. Here we give a summary of the categories of parameters that are available.

1) Specifications of the particular atom. Includes atomic weight, charge and effective charges, binding energies, population of electronic shells and refilling, and width of the ls state.

2) Data for the muonic states. Muon mass and energies of the muonic states are included; also selection of the initial distribution and population.

3) Choice of shells and multipolarities involved. Within the limitations explained earlier, any subshell and/or multipolarity can be considered or deleted.

4) Specifications for the x-ray catalogue. Includes high and low energy limits for the transition, intensity cutoff, transitions to be punched, and conversion of energy to channel number.

5) Parameters specifying the treatment of penetration. There are several ways of deciding if and where penetration is included in the computation.

6) Various bookkeeping options including commands to skip printing portions of the output, commands to calculate a case and to exit from the program.

Appendix $B$ shows excerpts from the input of a test run accompanying the writeup of ref. [64], and portions of the output from the same run.

5.4. Timing. The timing of the program will depend very strongly on two factors. a) The starting principal quantum number of the cascade plays a very significant role, since the timing depends on $n_{1}$ as a power between 4 and 5 thereof. b) The degree of inclusion of penetration is equally crucial; it takes a lot of time to calculate its contribution, since for each sub-rate calculated, at least one (and up to three) separate multipole like muonic matrix element has to be evaluated (which is a lengthy double sumation for high $n_{1}$ eccentric states). For the case illustrated the timing was just under 1 minute including compilation, loading and system time. The time would have been several 
minutes if penetration were included in its maximum allowed degree, with small changes in the outcome of the x-ray catalogue. To further elucidate the timing, we include a short table of typical runs with the associated times in table 5.1 below.

TABLE 5.1

Sample run times for test runs of the program.

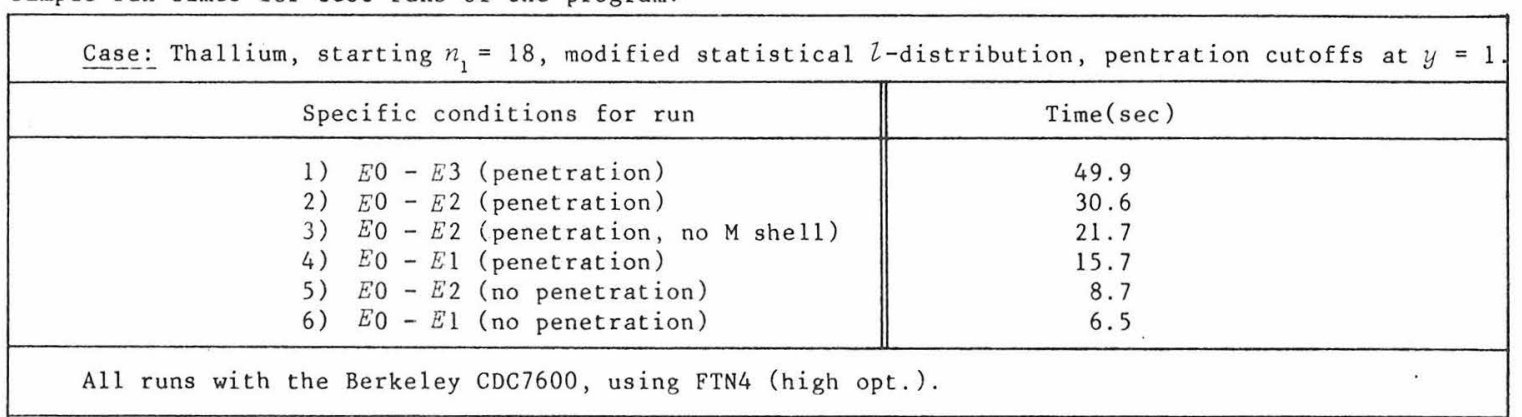

5.5. Practical Considerations and Limitations. Although this reworked version of the MUONIC ATOM CASCADE program takes into account many more phenomena than its predecessor, it is limited in part by the approximations stated in subsection 5.1 and in part by programming and execution limitations. To fully utilize the capabilities of the program with the minimum cost (if that is of importance), our experience has shown that a few shortcuts could be taken. Some points of interest for fitting of experimentally observed $x$-ray intensities follow.

1) Start the cascade a few $n_{1}^{\prime}$ s above the first transition to be fitted; $n_{1}=20$ is not necessary if, for example, the highest transition is from $n_{1}=13$. For a final fit, $n_{1}=20$ could be of interest.

2) One usually fits for the initial 2 -distribution; three options are available for that, plus the added option to fill the whole $(n, Z)$ spectrum.

3) In addition, the values of $Z^{*}$ and width of $K$ electron refilling could be adjusted slightly for a better fit, especially for low $Z$ atoms.

4) For most cases multipoles up to $E 2$ and all subshells are important, especially for the high part of the cascade.

5) Although penetration changes some rates dramatically, it can be eliminated in a variety of ways without serious consequences; some of the ways are

a) The leading term of penetration is adequate in most cases, the following terms contributing progressively less in the correction.

b) For $y \ll 1$ the penetration correction is small in all cases.

c) For small muon quantum numbers, Auger rates are small compared to radiative rates, and so penetration does not alter the results.

d) For the $3 d$ electrons the penetration can be skipped, since it is small in all cases.

6) Once the basic setup of a case is established, only the parameters to be changed have to be specified in multi-fit runs. 
CHAPTER 6: CONCLUSIONS

In the course of our research of the past few years we have studied the field of muonic atoms from their formation to their disintegration and the interaction of the muon with the surrounding electrons and the nucleus. In this thesis we have provided the missing link required to make a serious comparison with the experimental data. Although we have not formulated a theory that explains the data in a perfect way, we have pushed the problem one level higher, by taking into account several effects previously disregarded. Just as important as the primary objective of a cascade project are the intermediate results discovered. We have put the finger on several open questions and answered some; moreover we have identified some issues still unresolved, so that the next generation can try to resolve them in an effort to bring us closer to an accurate description of reality. As it stands now, we have a good knowledge of our achievements and limitations and the degree to which we can predict and/or explain the observables. We are confident that the researcher interested in pursuing the subject of muons in atoms further can find the information presented here and in our other publications useful as a starting reference. We hope that our basic stipulations will withstand the scrutiny of time. Our encouragement is passed to those who undertake the task of carrying the torch of knowledge to the future. 


\section{-51-}

APPENDIX A: MATHEMATICAL DETAILS AND COLlECtion OF FORMULAS

A.1. Multipole Radiation. In a radiative transition from state 1 to state 2 of multipolarity $E(L)$ (only electric type transitions considered here), the rate is given by [65]

$$
\Gamma_{\mathrm{R}}^{\mathrm{L}}=\frac{2(L+1)}{L[(2 L+1) ! !]^{2}} \alpha \omega\left(\frac{w \alpha}{z c}\right)^{2 L}\left|\int_{0}^{\infty} R_{2}^{*}(r)\left(\frac{r z}{a_{\mu}}\right)^{L} R_{1}(r) r^{2} \mathrm{~d} r\right|^{2} S\left(j_{1}, L, j_{2}\right)
$$

where $\omega$ is the transition frequency. We use for the dimensionless multipole integral

$$
I_{12}^{\mathrm{L}}=\left|\int_{0}^{\infty} R_{2}^{*}(r)\left(\frac{r^{\prime} z}{\alpha_{\mu}}\right)^{L} R_{1}(r) r^{2} \mathrm{~d} r\right|^{2} .
$$

The "statistical" factor $S\left(j_{1}, L, j_{2}\right)$ is given by

$$
S\left(j_{1}, L, j_{2}\right)=\frac{4 \pi}{2 L+1} \sum_{m_{1}} \sum_{M} \sum_{m_{2}}\left|\iint_{4 \pi} \Theta_{Z_{2} j_{2}}^{m_{2} *} Y_{L M}^{*} \Theta_{Z_{1} j_{1}}^{m_{1}} \mathrm{~d} \Omega\right|^{2},
$$

which works out to simply

$$
S\left(j_{1}, L, j_{2}\right)=\left(2 j_{2}+1\right)(2 L+1)\left(\begin{array}{ccc}
j_{1} & L & j_{2} \\
-\frac{1}{2} & 0 & \frac{1}{2}
\end{array}\right)^{2}
$$

Since the radial parts of the wave functions of the spin-orbit doublets are, in our non relativistic treatment, identical, the matrix element A.2 depends only on $l_{1}, l_{2}$ and not on $j_{1}, j_{2}$. Thus we do not want to treat $j$ explicitly, but rather $l$ (we will eventually divide the total $l_{1} \Rightarrow l_{2}$ rate into its various $j$ states according to their statistical weights). In such a case we may use a new statistical factor

$$
S\left(l_{1}, L, l_{2}\right)=\sum_{j_{2}=l_{2}-\frac{1}{2}}^{\tau_{2}+\frac{1}{2}} S\left(j_{1}, L, j_{2}\right)=\left(2 z_{2}+1\right)(2 L+1)\left(\begin{array}{lll}
l_{1} & L & z_{2} \\
& & \\
0 & 0 & 0
\end{array}\right)^{2} .
$$

Table A.l shows the radiative rates for the three lowest multipolarities.

A.2. Relative Intensities of Spin Multiplets. Assuming that the initial states are populated proportionally to their statistical weight, $i . e ., \propto\left(2 j_{1}+1\right)$, the relative intensity of the transition $\left(l_{1}, j_{1}\right) \rightarrow\left(z_{2}, j_{2}\right)$ with respect to the total transition $l_{1} \Rightarrow l_{2}$ is given by

$$
\frac{\left(2 j_{1}+1\right) S\left(j_{1}, L, j_{2}\right)}{\left(2 l_{1}+1\right) S\left(l_{1}, L, l_{2}\right)}=2(2 L+1) \frac{\left(2 j_{1}+1\right)\left(2 j_{2}+1\right)}{\left(2 l_{1}+1\right)\left(2 l_{2}+1\right)} \frac{\left(\begin{array}{ccc}
j_{1} & L & j_{2} \\
-\frac{1}{2} & 0 & \frac{1}{2}
\end{array}\right)^{2}}{\left(\begin{array}{ccc}
Z_{1} & L & l_{2} \\
0 & 0 & 0
\end{array}\right)^{2}} .
$$

Table A.2 shows the unnormalized relative intensities up to octupole transitions, in reference to eq. A.6. Note that the same relations hold for the relative intensities of Auger transitions.

A.3. Multipole Auger Transitions (no Penetration). In an Auger transition of multipolarity $L$ described schematically by 
Initial

$\begin{array}{ll}\text { Muon } & n_{1}, l_{1}, j_{1} \\ \text { Electron } & n^{\prime}, l^{\prime}, j^{\prime} \text { (bound) }\end{array}$

\section{Final}

$n_{2}, l_{2}, j_{2}$.

$k, l, j$ (continuum)

the transition rate is given by [42]

$$
\Gamma_{\mathrm{A}}^{\mathrm{L}}=\left\{\left|\frac{e^{2}}{\hbar} \iint \psi_{\mu 2}^{*}\left(r_{1}\right) \psi_{\epsilon 2}^{*}\left(r_{2}\right) \frac{\mathrm{e}^{-i K r_{12}}}{r_{12}} \psi_{\mu 1}\left(r_{1}\right) \psi_{\mu_{1}}\left(r_{2}\right) \mathrm{d}^{3} r_{1} \mathrm{~d}^{3} r_{2}\right|^{2}\right\} \begin{aligned}
& \text { mult. } L \\
& \text { part }
\end{aligned}
$$

To obtain the various multipolarities, we expand $1 / x_{12}$ in spherical harmonics (using, for the time being, the long wave length approximation $e^{i K r_{12}} \approx 1$ ). The rate then becomes

$$
\begin{aligned}
\Gamma_{\mathrm{A}}^{L}= & \frac{e^{4}}{\hbar^{2}}\left|\int_{0}^{\infty} \int_{0}^{\infty} R_{\mu 2}^{*}\left(r_{1}\right) R_{e 2}^{*}\left(r_{2}\right) \frac{r_{<}^{L}}{r_{>}^{L+1}} R_{\mu 1}\left(r_{1}\right) R_{e 1}\left(r_{2}\right) r_{1}^{2} \mathrm{~d} r_{1} r_{2}^{2} \mathrm{~d} r_{2}\right|^{2} \\
& \times \mid \text { Angular Part }\left.\right|^{2} \times \quad \text { (Number of electrons present in subsheli) },
\end{aligned}
$$

where we have multiplied by the number of electrons present in a particular subshell. At the end we will have to add the contributions of all final electronic states $(\mathcal{l}, j)$, since they are normally not observed. The angular part is separable (if one treats $l$ only, disregarding the various $j$. states) into an electronic and a muonic part, each similar to that of radiation, that is

$$
\text { Angular Part }\left.\right|^{2}=\frac{\left(2 l_{2}+1\right)(2 l+1)}{(2 L+1)}\left(\begin{array}{lll}
l_{1} & L & l_{2} \\
0 & 0 & 0
\end{array}\right)^{2}\left(\begin{array}{lll}
l^{\prime} & L & l^{2} \\
& & \\
0 & 0 & 0
\end{array}\right)^{2}
$$

Table A. 3 shows the angular parts for $L=0,1,2$, and 3 . The case of $L=0$ is given in anticipation of the penetration, which permits the otherwise forbidden monopole transitions. Note that in the formulation of [42] the continuum wave functions are normalized as follows

$$
\psi_{e 2}\left(r_{2}\right)=\sqrt{\frac{m_{e}}{\hbar}} 2^{2+1} \kappa^{Z+\frac{1}{2}} e^{i k r_{2}} r_{2}^{2} F_{1} F_{1}\left(z+1-i y ; 2 l+2 ;-2 i k r_{2}\right)|\Gamma(z+1-i y)| e^{\frac{1}{2} \pi y} \frac{y_{l m}\left(\Omega_{2}\right)}{(2 l+1) !}
$$

As a first approximation, we will assume that the muon moves completely inside the electron orbit, and therefore $r_{<} \equiv r_{1}$ and $r_{>} \equiv r_{2}$. In such a case, the two integrals of (A.9) decouple. The muonic part is the multipole matrix element $I_{12}$ (the same one as in radiation). The electronic part is more complicated, but it amounts in performing integrations of the general form

$$
\int_{0}^{\infty} r_{2}^{M} \mathrm{e}^{-\xi r_{2}} F_{1}\left(a ; b ; c r_{2}\right)=\frac{\Gamma(M+1)}{\xi^{M+1}} 2_{1} F_{1}(a, M+1 ; b ; c / \xi),
$$

and combinations thereof. The procedure for evaluating such integrals is described in ref. [42]; they yield Jacobi (hypergeometric) polynomials, which can be evaluated exactly in terms of elementary functions. For the more involved cases we used the algebraic manipulating computer language REDUCE-2 [66]. Tables A.4, A.5, and A.6 show the compilation of all these rates classified by multipolarity and subshell. 
A.4. Retardation and Electronic Screening Effects in Auger Transitions. The exponential factor $\exp \left(i K r_{12}\right)$ in eq. A.8 represents the effect of retardation. To take it into account we use the expansion [29]

$$
\left.\frac{e^{i K r_{12}}}{r_{12}}=4 \pi i K \sum_{L=0}^{\infty} j_{L}\left(K r_{<}\right) h_{L}^{\left(1 \xi_{K}\right.}\right) \sum_{M=-L}^{L} Y_{L M}^{*}\left(\Omega_{1}\right) y_{L M}\left(\Omega_{2}\right) .
$$

Using as before $r_{<} \equiv r_{1}, r_{>} \equiv r_{2}$ and assuming $K r_{1} \ll 1$ one can safely use the leading term in the spherical Bessel function expansion

$$
j_{L}\left(K r_{1}\right) \approx \frac{\left(K r_{1}\right)^{L}}{(2 L+1) ! !}
$$

The muonic part of the transition matrix element is unchanged, while the operator in the electronic part is modidified to

$$
\frac{1}{r_{2}^{L+1}} \Rightarrow \frac{1}{r_{2}^{L+1}}\left[1+\frac{1}{2(2 L-1)} K^{2} r_{2}^{2}\right]
$$

Another possible modification is to use a Yukawa type potential, viz.

$$
\frac{1}{r_{12}} \rightarrow \frac{\mathrm{e}^{-\lambda r_{12}}}{r_{12}}
$$

to reflect the adiabatic screening of the muon charge by the electron cloud [67]. The screening parameter $\lambda$ is related to the density of the electron Fermi gas $\rho$ by

$$
\lambda=\left\{\frac{6 \pi \rho e^{2}}{\varepsilon_{F}}\right\}^{\frac{1}{2}} .
$$

For both modifications the calculational techniques for evaluating the electronic integrals are similar to the ones used for the original unmodified integrals. The resulting modifications for a limited selection of cases is shown in table A.7; the importance of these results is discussed in chapter 2.

A.5. Penetration Effects in Auger Transitions. To evaluate the radial part of the matrix element (A.9) exactly, we have the choice of performing the electronic or the muonic integration first. Both possibilities have been investigated and each has its own merits. We will first discuss briefly the "exact" method (but otherwise inefficient).

A.5.1. Muonic Integrals Done First. The first (inside) integral is always incomplete, since the functional form of the integrand changes at $r_{1}=r_{2}$. In this case, we expand the Laguerre polynomials appearing in the muonic wave functions in power series (finite). The resulting integrated functions are incomplete gamma functions, which in turn are expanded in finite power series. Then the complete integrals over the electronic wave functions involve products of powers, exponentials and confluent hypergeometric functions (Kummer functions). According to the general procedure of ref. [42] the integrations yield Gauss hypergeometric functions, of relatively complicated indices and argument; they, in turn, can be expanded in power series. The result is that in order to evaluate one matrix element it is necessary to perform four interlaced summations, two due to the Laguerre polynomials and one each due to the resulting incomplete gamma functions and for the expansion of the final 
hypergeometric functions. In principle, this method is exact, but in practice it takes too long to evaluate and it is subject to severe numerical instabilities, because of the extreme cancellation taking place. Having failed to produce a usable algorithm, we resort to performing the integrals in the reverse order.

A.5.2. Electronic Integrals Done First. Rewriting the integrals of (A.9) in a manner exemplifying the contribution of penetration, and using the orthogonality properties of the radial parts of the electronic wave functions we obtain for the radial double integral of (A.9) the following formula

$$
\begin{gathered}
\int_{0}^{\infty} R_{\mu 2}^{*}\left(r_{1}\right) r_{1}^{L} R_{\mu_{1}}\left(r_{1}\right) r_{1}^{2} \mathrm{~d} r_{1} \int_{0}^{\infty} R_{e_{2}}^{*}\left(r_{2}\right) \frac{1}{r_{2}^{L+1}} R_{e_{1}}\left(r_{2}\right) r_{2}^{2} \mathrm{~d} r_{2} \\
-\int_{0}^{\infty} R_{\mu 2}^{*}\left(r_{1}\right) R_{\mu 1}\left(r_{1}\right) r_{1}^{2} \mathrm{~d} r_{1}\left\{\int_{0}^{r} R_{e_{2}}^{*}\left(r_{2}\right)\left[\frac{r_{1}^{L}}{r_{2}^{L+1}}-\frac{r_{2}^{L}}{r_{1}^{L+1}}\right] R_{e_{1}}\left(r_{2}\right) r_{2}^{2} \mathrm{~d} r_{2}\right\},
\end{gathered}
$$

where the first line is the part without penetration evaluated previously in sect. A. 3 and. the second line is the contribution from penetration. To evaluate the penetration we note that if we can express the function in the braces in a convenient form, the complete muonic integrals could be done without the complications of sect. A.5.1. Note also that we deal with a limited number of electronic states and multipolarities (except for the continuous momentum of the free electron), so that we could make a table of these functions, which could be used with the muonic states. Since $R_{21}\left(r_{2}\right)$ involves only powers and exponentials of $r_{2}$, we could study the case of

$$
R_{e 1}\left(r_{2}\right) \propto \sum_{M=z^{\prime}}^{n^{\prime}-1} C_{n^{\prime} \tau^{\prime} M} r^{M} \exp \left\{-\frac{r_{2} z}{n^{\prime} a_{0}}\right\} \text {, }
$$

where $C_{n^{\prime} Z^{\prime} M}$ are proportional to the Laguerre coefficients of the electronic state ( $n^{\prime}, Z^{\prime}$ ), being defined (normalized wave functions) as [42]

$$
C_{n^{\prime} Z^{\prime} M}=\left\{\frac{\left(n^{\prime}-Z^{\prime}-1\right) !\left(n^{\prime}+Z^{\prime}\right) !}{2 n}\right\}^{\frac{1}{2}}\left(\frac{2 Z}{n^{\prime} a_{0}}\right)^{M+\frac{3}{2}}(-1)^{M-Z^{\prime}} \frac{1}{\left(n^{\prime}-1-M\right) !\left(l^{\prime}+1+M\right) !\left(M-Z^{\prime}\right) !} .
$$

It is therefore sufficient to study one term of the type of (A.19), the general one being formed by linear combinations thereof. Noting the functional form (A.ll) of the continuum wave function and using the definition of the parameter $y$

$$
y=\frac{2^{*} \alpha}{\sqrt{2 \hbar \kappa / m_{e} c^{2}+\hbar^{2} \kappa^{2} / m_{e}^{2} c^{4}}},
$$

we can retain only the variable dependence of the function in braces (A.18) and in light of (A.19) we want to study the function (from here on $r$ is measured in units of $a_{0} / Z$ )

$$
g\left(r_{1}\right)=\int_{0}^{r_{1}} r_{1}^{M} \mathrm{e}^{-r_{2} / r^{\prime}}\left[\frac{r_{1}^{L}}{r_{2}^{L+1}}-\frac{r_{2}^{L}}{r_{1}^{L+1}}\right] r_{1}^{z^{\prime}} \mathrm{e}^{-i k r_{2}}{ }_{1} F_{1}\left(z^{\prime}+1+i y ; 2 z^{\prime}+2 ; 2 i k r_{1}\right) .
$$

To single out the y dependence of this function (and therefore end up with a "universal" expression 
depending only on the integers $M, n^{\prime}, L$, and $Z^{\prime}$ ), we expand the confluent hypergeometric function in terms of Bessel functions [68]

$$
\begin{aligned}
& { }_{1} F_{1}(a ; c ; z)=\Gamma(c) e^{\frac{1}{2} z}\left(\frac{1}{2} c z-a z\right)^{\frac{1}{2}-\frac{1}{2} c} \sum_{j=0}^{\infty} A_{j}\left(\frac{1}{2} z\right)^{\frac{1}{2} j}(c-2 a)^{-\frac{1}{2} j} J_{c+j-1}(\sqrt{2 c z-4 a z}), \\
& \text { where } A_{0}=1, A_{1}=0, A_{2}=\frac{1}{2} c \text { and } A_{j}(j>2) \text { are derived from the recursion: } \\
& (n+1) A_{n+1}=(n+c-1) A_{n-1}+(2 a-c) A_{n-2}
\end{aligned}
$$

After working out the arithmetic and assuming that the series is rapidly converging, we obtain for the first few terms in the expansion of

$$
g\left(r_{1}\right)=\frac{\left(2 l^{\prime}+1\right) !}{2^{Z^{\prime}+\frac{1}{2}}} \sum_{j=0}^{\infty}\left\{\begin{array}{cc}
1 & ; j=0 \\
0 & ; j=1 \\
-\frac{l^{\prime}+1}{y^{2}} & ; j=2 \\
\frac{1}{3} \frac{1}{y^{2}} & ; j=3
\end{array} \int_{0}^{r_{1}^{1}} r_{2}^{\frac{1}{2}(j-1)+M} \mathrm{e}^{-r_{2} / n^{\prime}} J_{2 l^{\prime}+1+j}\left(2 \sqrt{2 r_{2}}\right)\left[\frac{r_{1}^{L}}{r_{2}^{L+1}}-\frac{r_{2}^{L}}{r_{1}^{L+1}}\right] \mathrm{d} r_{2} \cdot\right.
$$

Our strategy is to fit the integral in (A.24) by a convenient function. Since the muonic integral involves powers and exponentials, a candidate would be a combination of terms of the form

$$
\text { Integral } \approx \beta r_{1}^{N} \mathrm{e}^{-\alpha r_{1}},
$$

where $N$ is an integer and the coefficients $\beta$ and $\alpha$ depend on the indices $\tau^{\prime}, M, L, n^{\prime}$, and $j$. $I f$ we consider the case of small $r_{1}$ (and therefore $r_{2}$ ) we can expand the Bessel function in power series and get the leading behavior of the integral. It turns out that for such a case

$$
N=l^{\prime}+M+j+2 \geq 2 .
$$
Using this fact and only one term of the type (A.25) we were able to fit the first three nonzero terms in (A.24) for all cases of interest, and obtain quantities $\beta$ and $\alpha$. The fit was concentrated in a range of $r_{1}$ where the muonic wave functions are substantially different from zero. Typically the fits are $0.5 \%$ accurate (maximum deviation) for muonic wave functions up to $n=20$. The fitted values $\beta$ are very close to the exact values of the limit of small $r_{1}$ and $\alpha$ is in the 0.003 region, for the modified muonic matrix elements in the second line of (A.18), in units such that the maximum contribution to the muonic matrix element is from the region $r_{1} \sim n^{2}, n \sim n_{1}, n_{2}$.

Having evaluated $g\left(r_{1}\right)$, the outside integral of the penetration in (A.18) is a modified multipole matrix element, the operator being a combination of an integer power and an exponential. The numerical methods of evaluating such a matrix element are very analogous to those of the genuine multipole matrix element. Finally we have to assemble the term by combining the various parts of (A.19), subtract it from the integral without penetration, and proceed as usual. There are 162 pairs of fitted numbers $\beta$ and $\alpha$, a table too long and cumbersome to report here; the values of the fitted coefficients can be obtained from the data of the cascade program [64]. The full impact of this modification is discussed in detail in chapter 2 . 
Figure A.1

Comparison of the fitted and exact penetration functions.

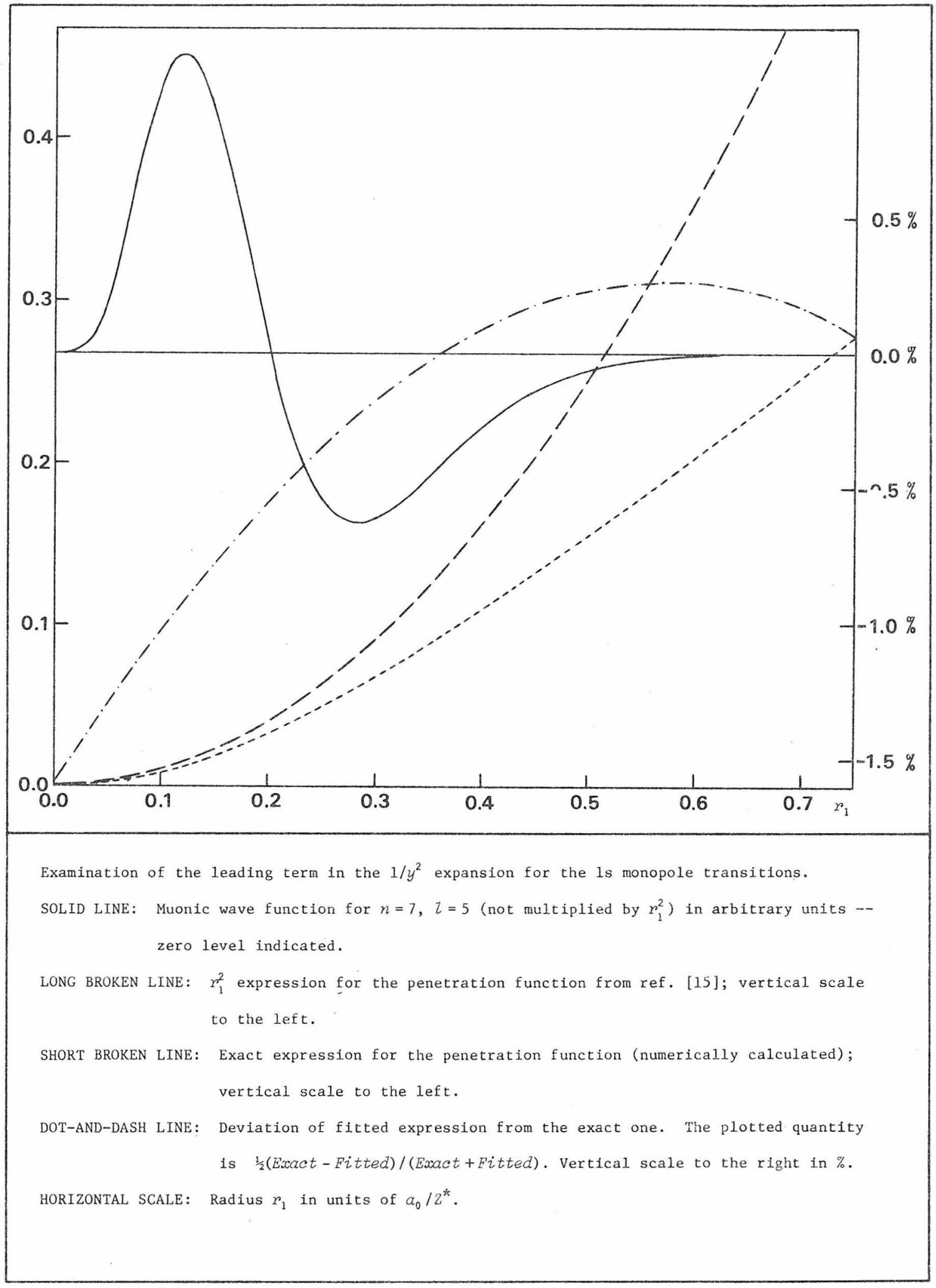


TABLE A.1

Multipole radiative rates.

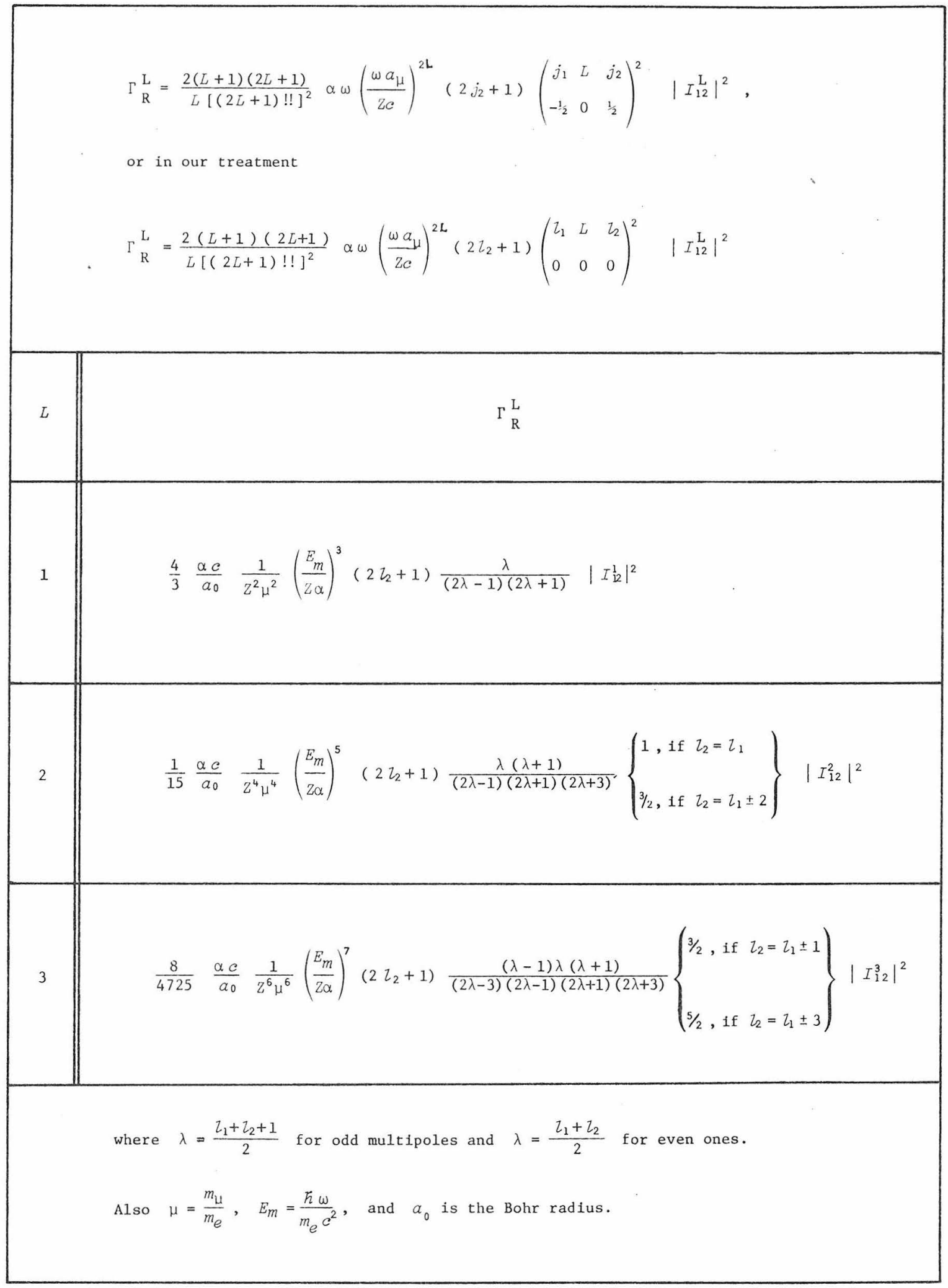


TABLE A.2

Relative intensities of radiative and Auger transitions.

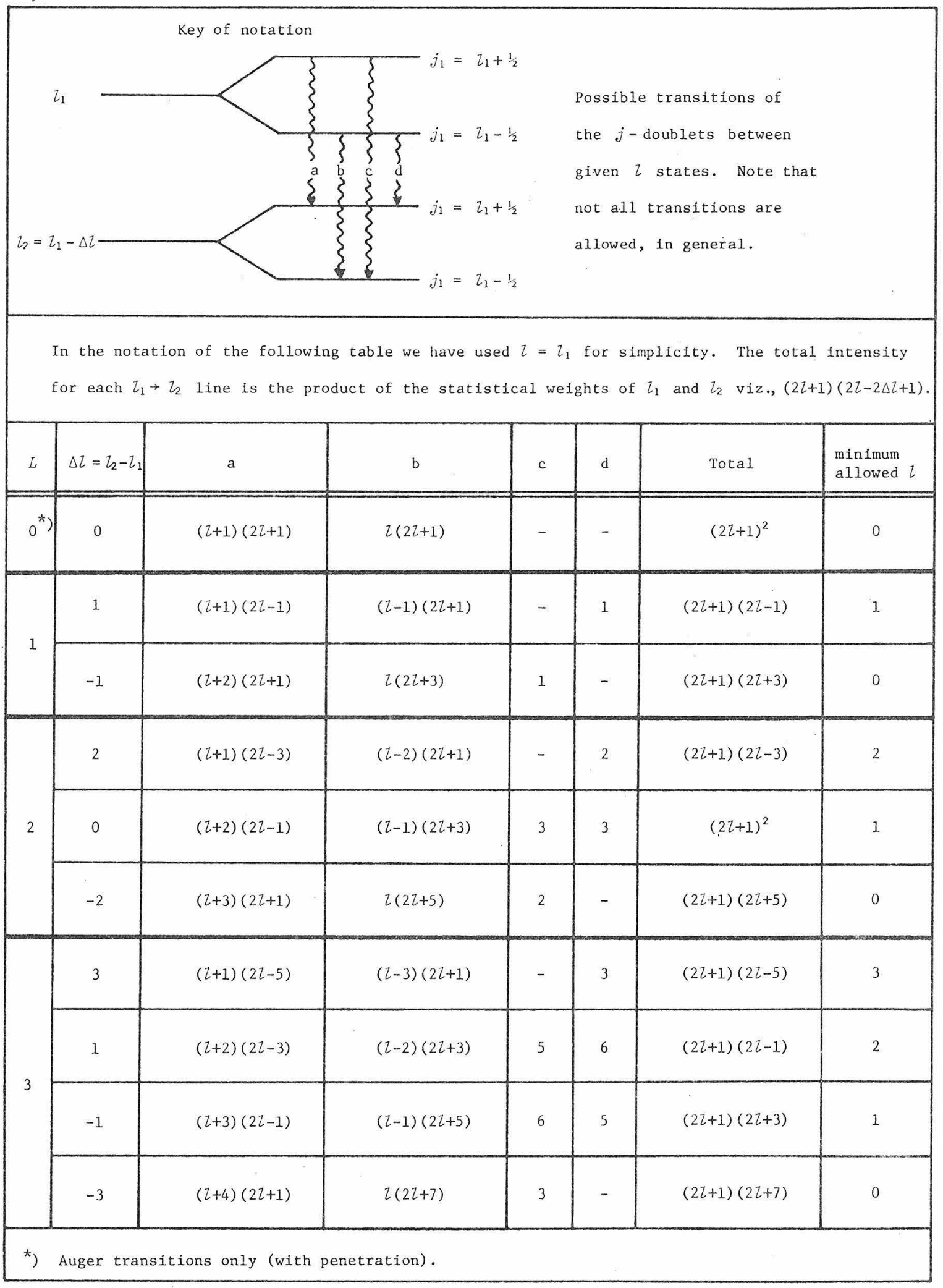


TABLE A. 3

Angular parts of multipole Auger transitions.

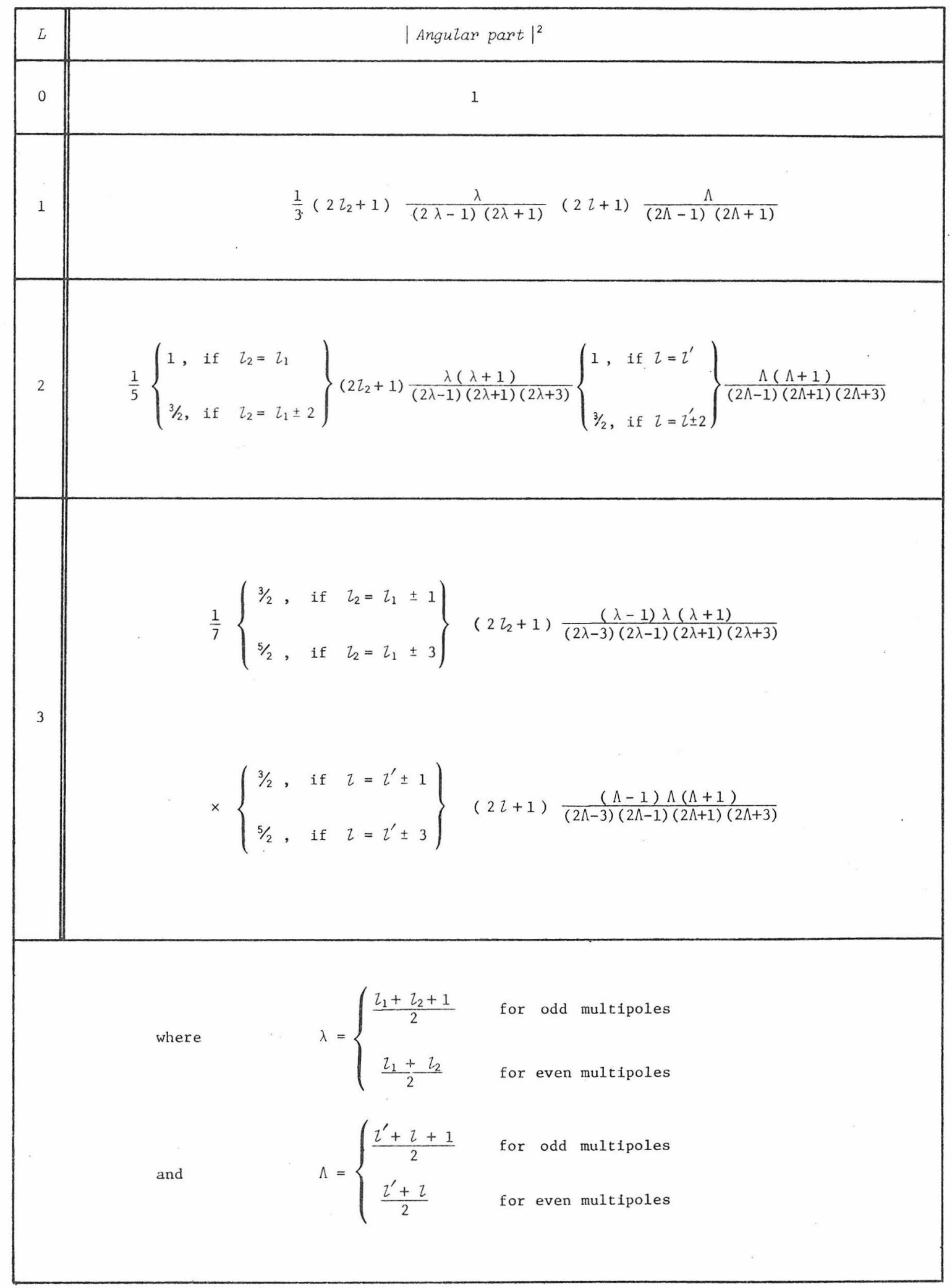


TABLE A. 4

Dipole Auger rates (no penetration)

\begin{tabular}{|c|c|}
\hline \multirow{2}{*}{\multicolumn{2}{|c|}{$\begin{array}{l}\text { In this and the following two tables, the rates are shown for fully occupied electronic } s \\
\text { Also we use the notation } P_{n}=\exp \left\{y\left[2 \tan ^{-1}\left(\frac{y}{n}\right)-\pi\right]\right\} \text {. } \\
\qquad \mathrm{r}_{\mathrm{A}}^{\mathrm{L}=1}=\frac{2}{3} \frac{\pi \alpha c}{\alpha_{0}}\left(2 \zeta_{2}+1\right) \frac{\lambda}{(2 \lambda-1)(2 \lambda+1)} \frac{\mathrm{e}^{\pi y}}{\sinh (\pi y)}\left(\frac{z^{*}}{2}\right)^{2}\left|I_{12}^{1}\right|^{2} \times \text { (Multiplier) }\end{array}$}} \\
\hline & \\
\hline $\begin{array}{c}\text { Electron } \\
\text { state }\end{array}$ & Multiplier \\
\hline 1s & $16 \frac{y^{2}}{1+y^{2}} P_{1}^{2}$ \\
\hline $2 \mathrm{~s}$ & $32 \frac{y^{2}\left(1+y^{2}\right)}{\left(4+y^{2}\right)^{2}} P_{2}^{2}$ \\
\hline $2 \mathrm{p}$ & $8 \frac{y^{4}\left(12+11 y^{2}\right)}{\left(4+y^{2}\right)^{3}} P_{2}^{2}$ \\
\hline $\begin{array}{l}L-\text { shel1 } \\
\text { tota1 }\end{array}$ & $8 \frac{y^{2}\left(4+3 y^{2}\right)\left(4+5 y^{2}\right)}{\left(4+y^{2}\right)^{3}} P_{2}^{2}$ \\
\hline $3 \mathrm{~s}$ & $\frac{16}{3} \frac{y^{2}\left(1+y^{2}\right)\left(27+7 y^{2}\right)^{2}}{\left(9+y^{2}\right)^{4}} P_{3}^{2}$ \\
\hline $3 p$ & $\frac{128}{3} \frac{y^{4}\left(81+96 y^{2}+19 y^{4}\right)}{\left(9+y^{2}\right)^{4}} P_{3}^{2}$ \\
\hline 3d & $\frac{256}{3} \frac{y^{6}\left(1+y^{2}\right)\left(45+11 y^{2}\right)}{\left(9+y^{2}\right)^{5}} P_{3}^{2}$ \\
\hline $\begin{array}{c}\text { M- shell } \\
\text { total }\end{array}$ & $\frac{16}{3} \frac{y^{2}\left(81+78 y^{2}+13 y^{4}\right)\left(81+126 y^{2}+29 y^{4}\right)}{\left(9+y^{2}\right)^{5}} P_{3}^{2}$ \\
\hline
\end{tabular}


Quadrupole Auger rates (no penetration)

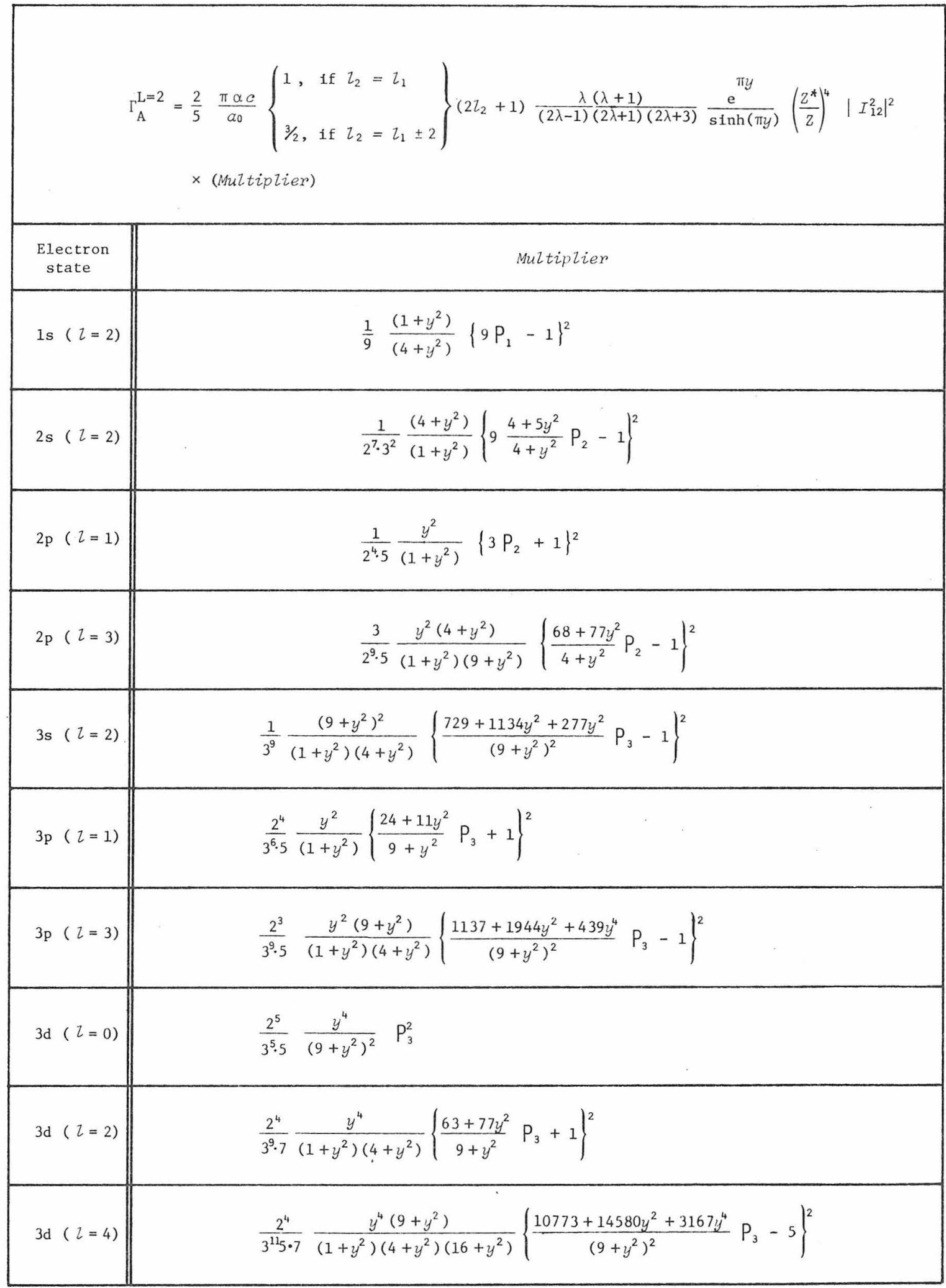


Octupole Auger rates (no penetration)

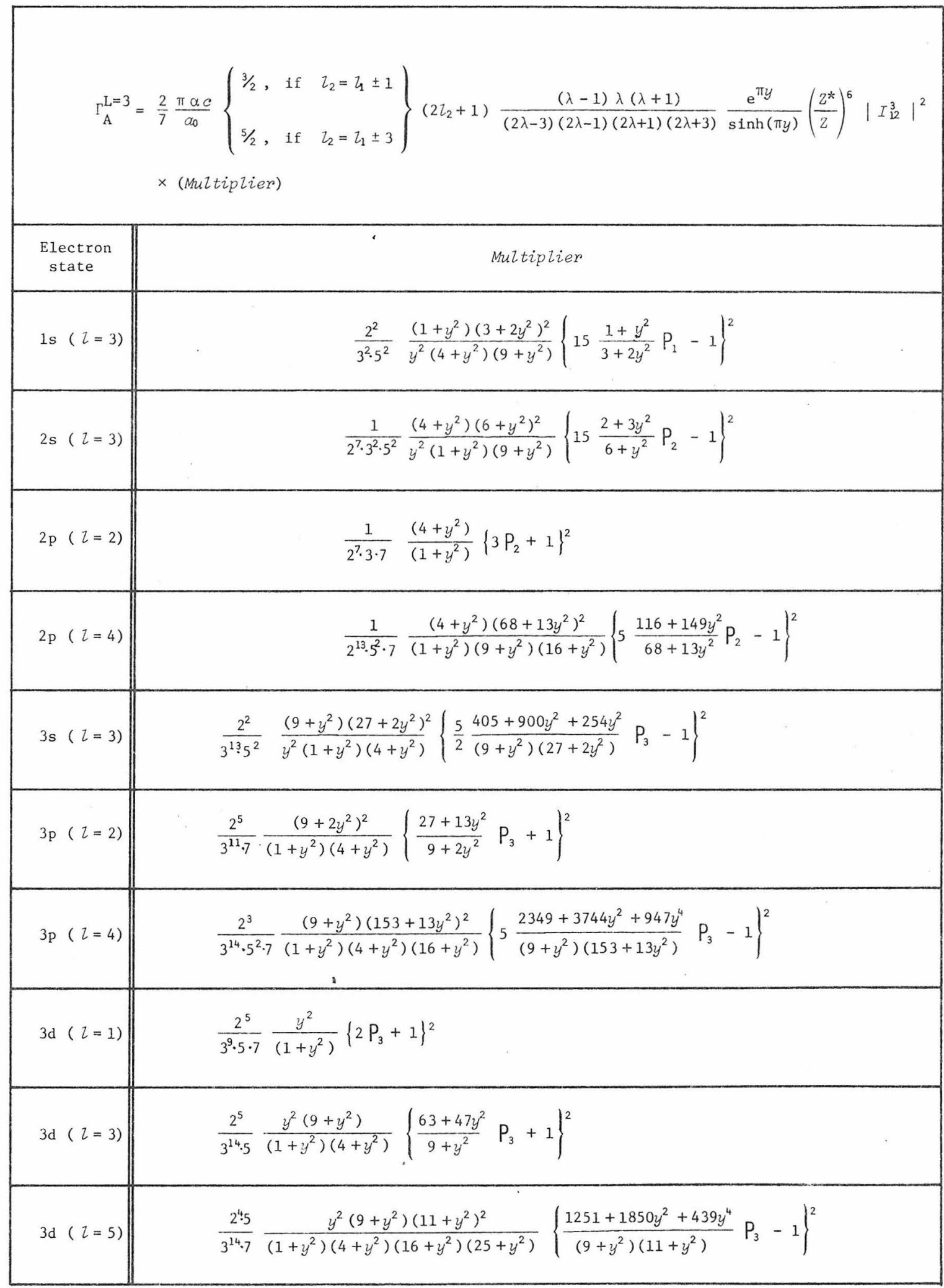


TABLE A. 7

Selected results for retardation and electronic screening.

Only the portion of the rate formula which is affected is given; the rest of the factors stay intact.

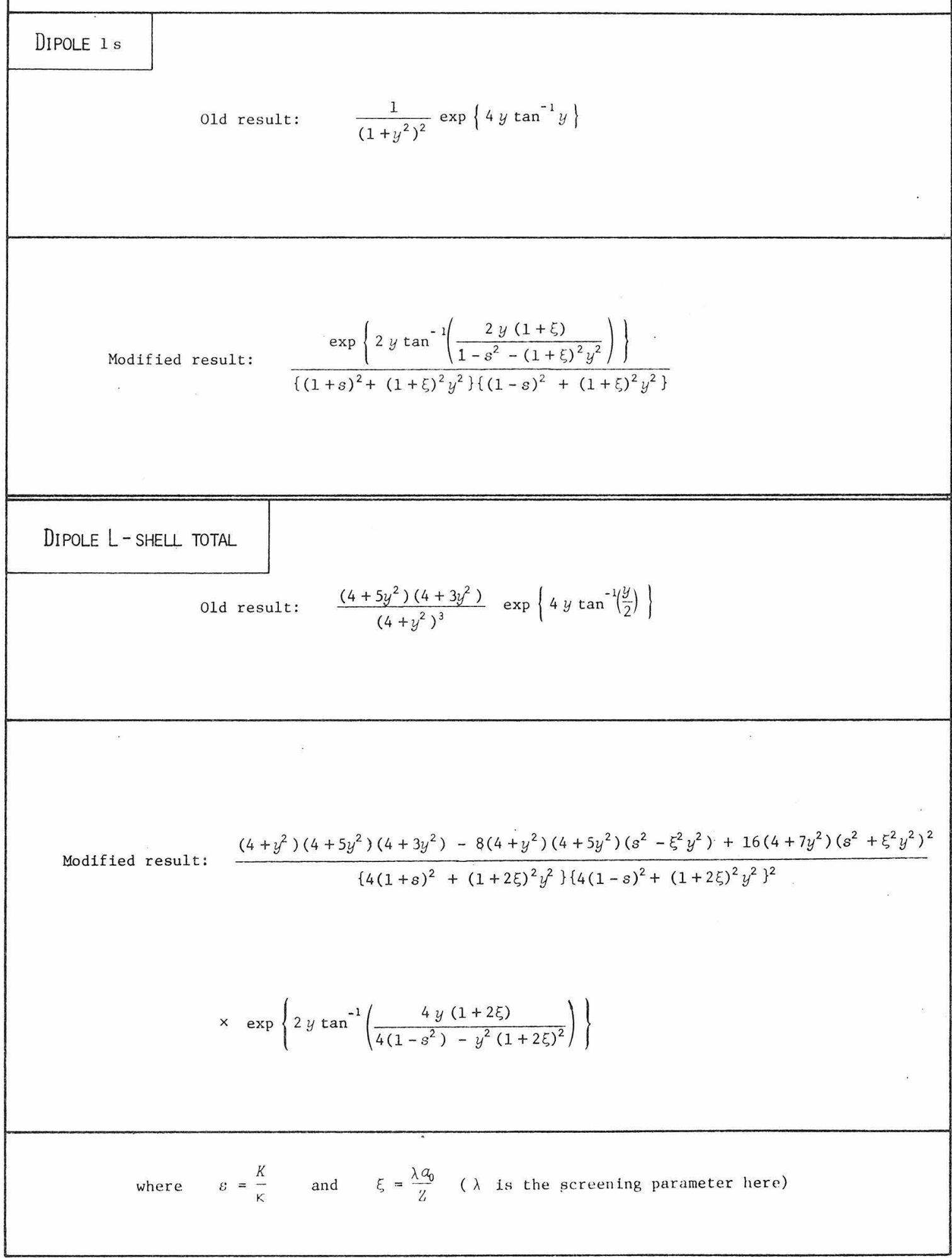


APPENDIX B: SAMPLE INPUT AND OUTPUT OF THE MUONIC ATOM CASCADE PROGRAM

To illustrate some key features of the program and the diversity of option available to the user, we have reproduced portions of our test run. The first page illustrates the input, while the next three show key pages of the output.

EXPLANATIONS OF THE PAGES REPRODUCED.

PAGE B.1: This is part of the sample input; some cards involving the transitions to be punched have been removed.

PAGE B.2: This is a page that shows at a glance the values and defaults of all parameters used in the program; it could be used as a title sheet, if so desired.

PAGE B.3: This page is of interest if one wants to see why some features exist in the cascade; it summarizes important quantities, as populations and widths.

PAGE B.4: This is the final page of the table of intensities; the transitions are identified by quantum numbers and energy, and a channel number is given if requested. 
$-65$

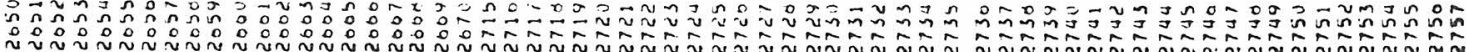

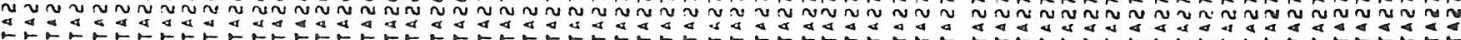

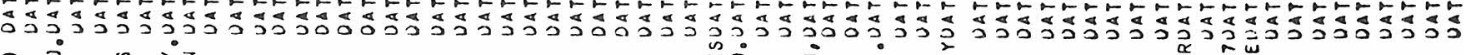

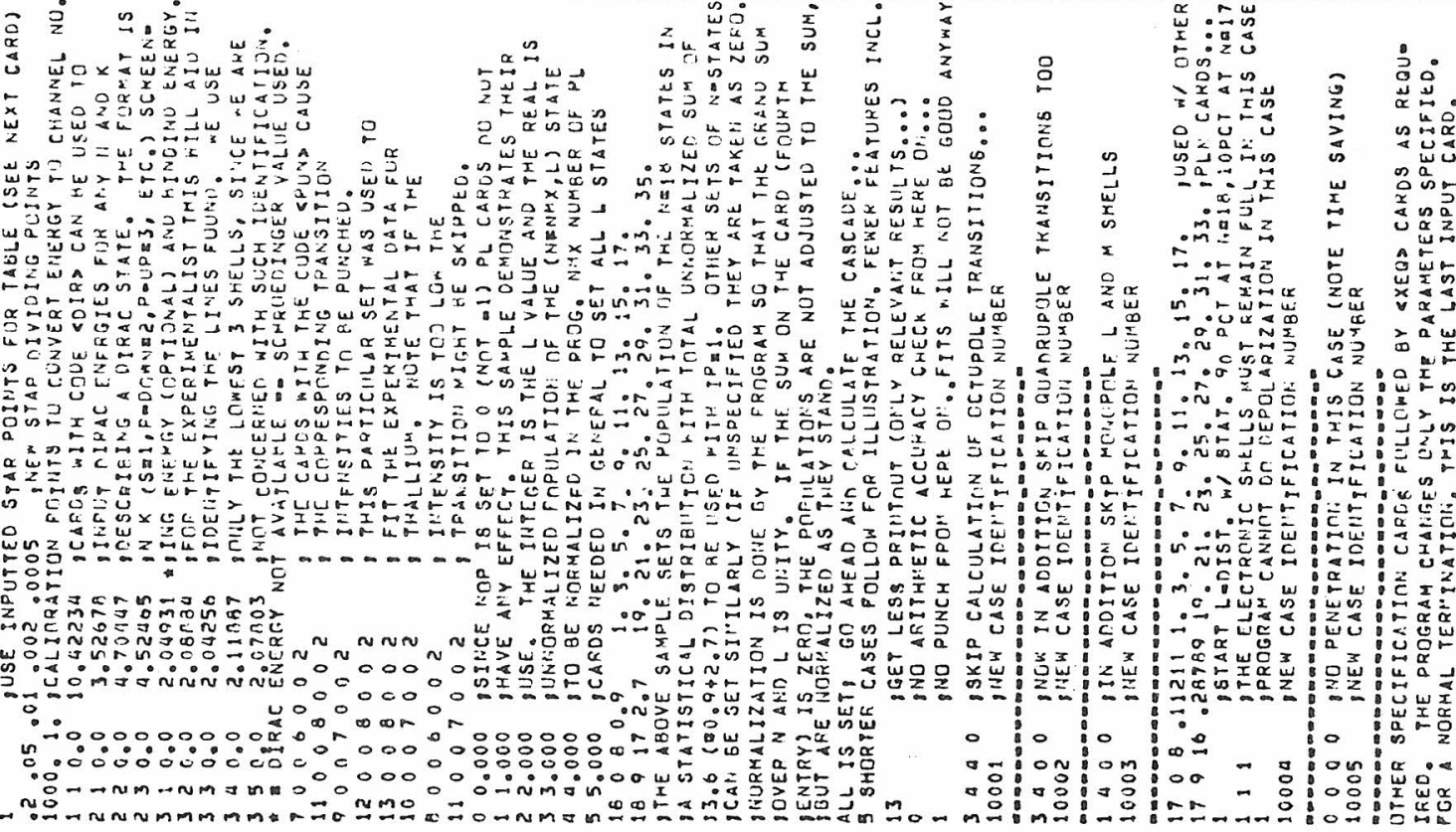

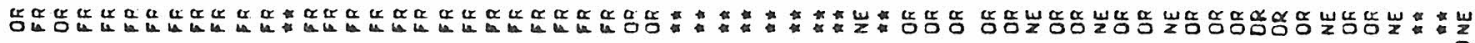

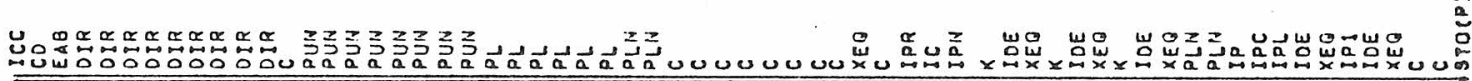

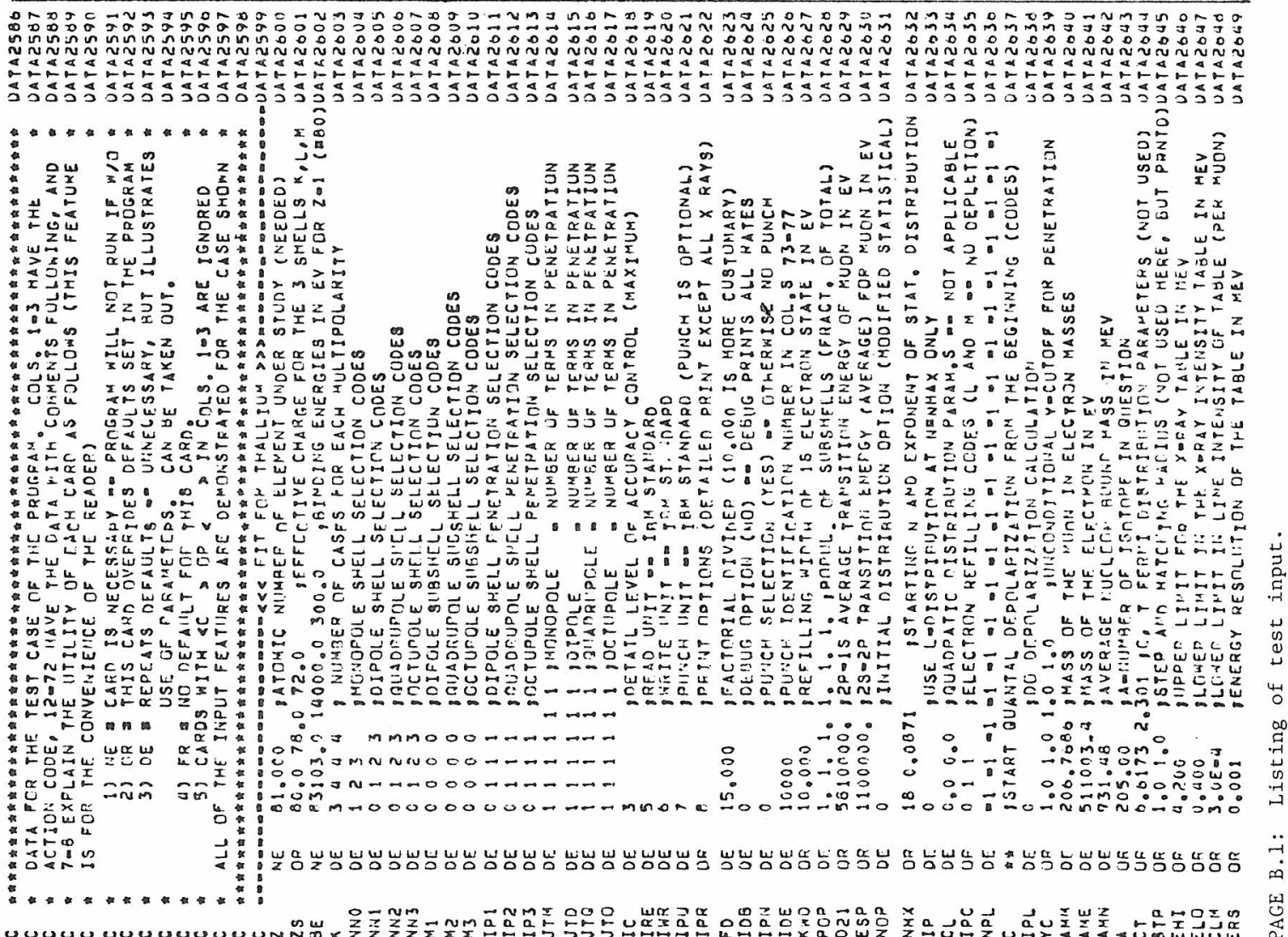


PAGE B.2: Summary of input parameters.

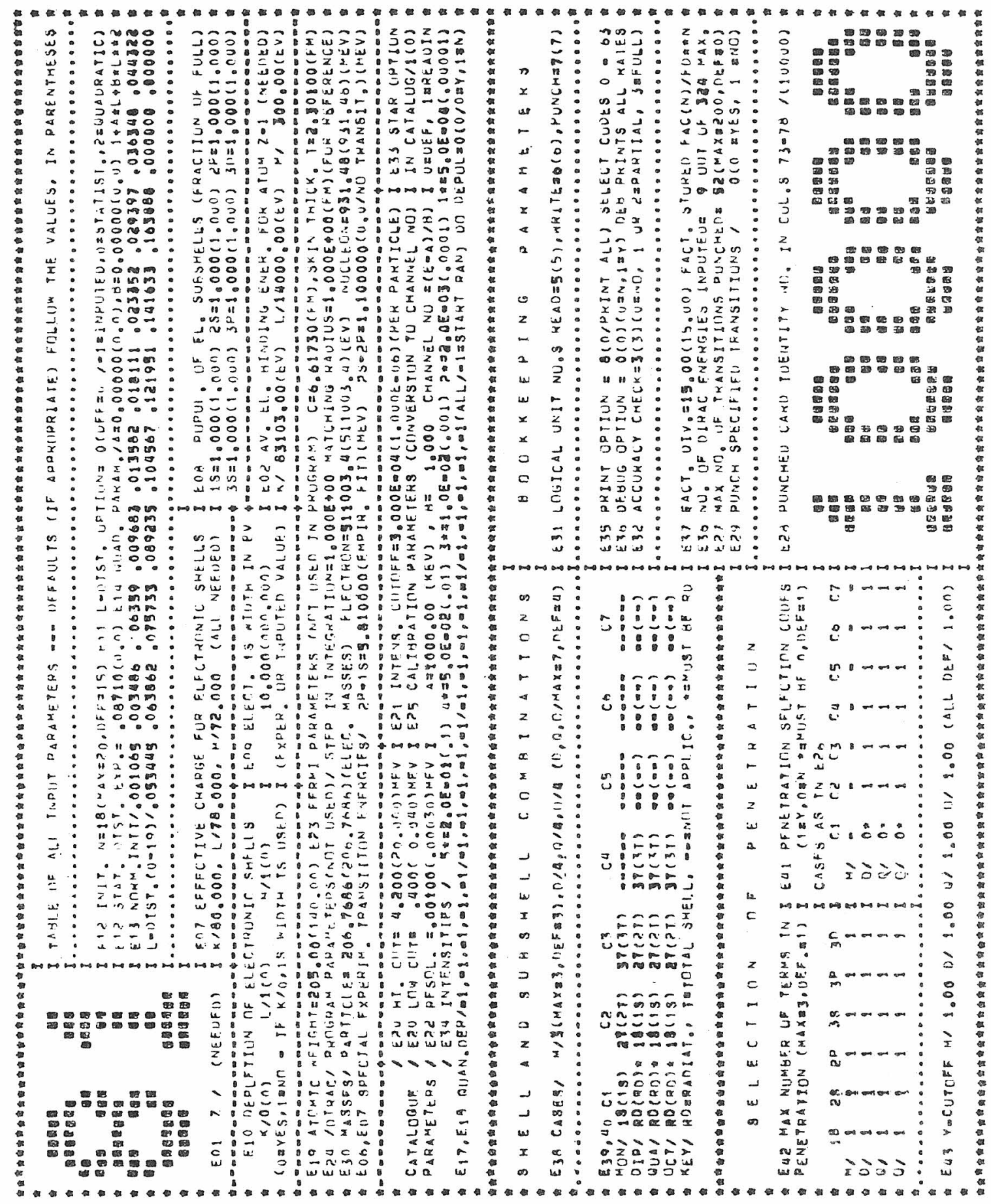


PAGE B.3: Summary of the results at the end of the cascade (last page).

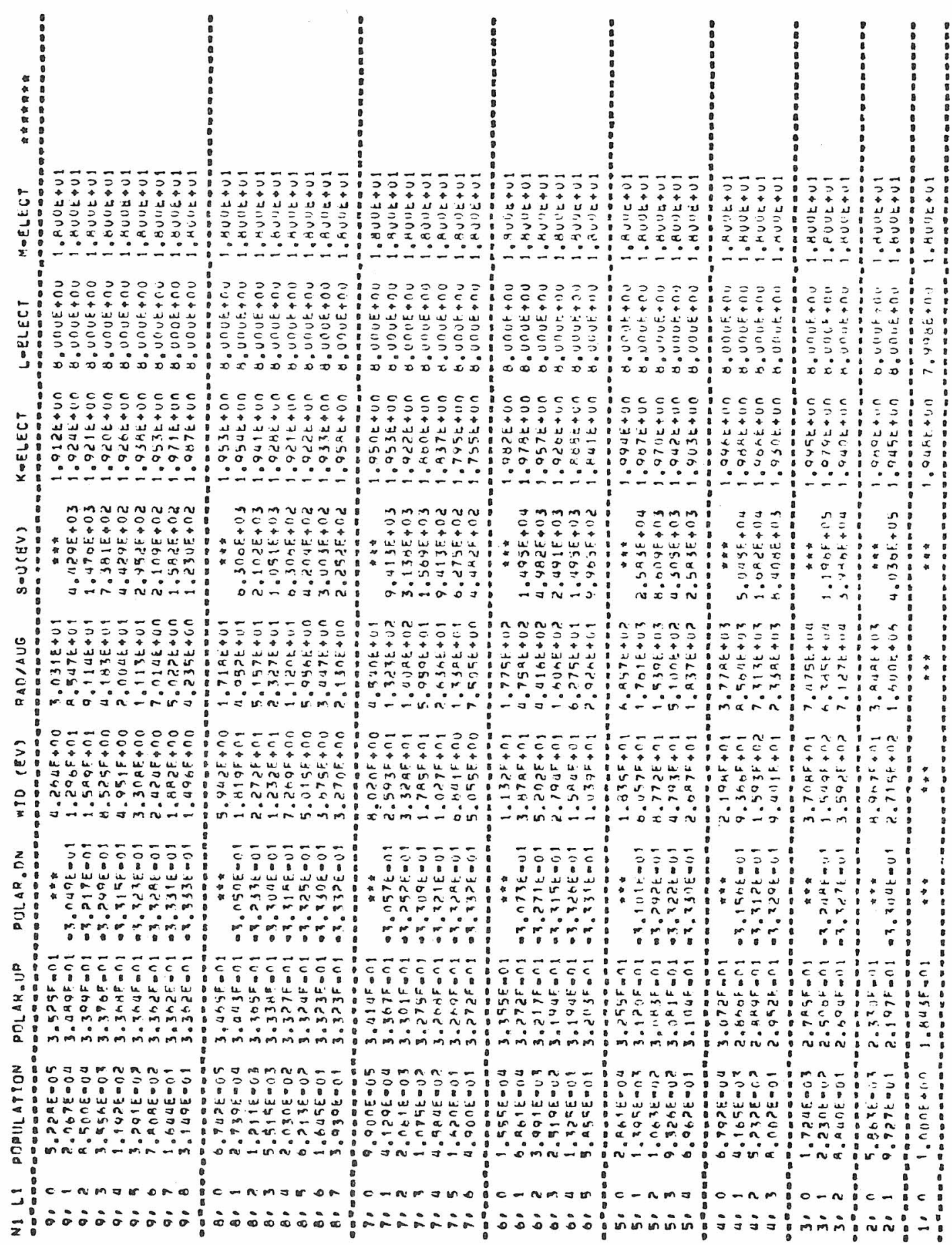


PAGE B.4: Catalogue of $x$-ray intensities (last page).

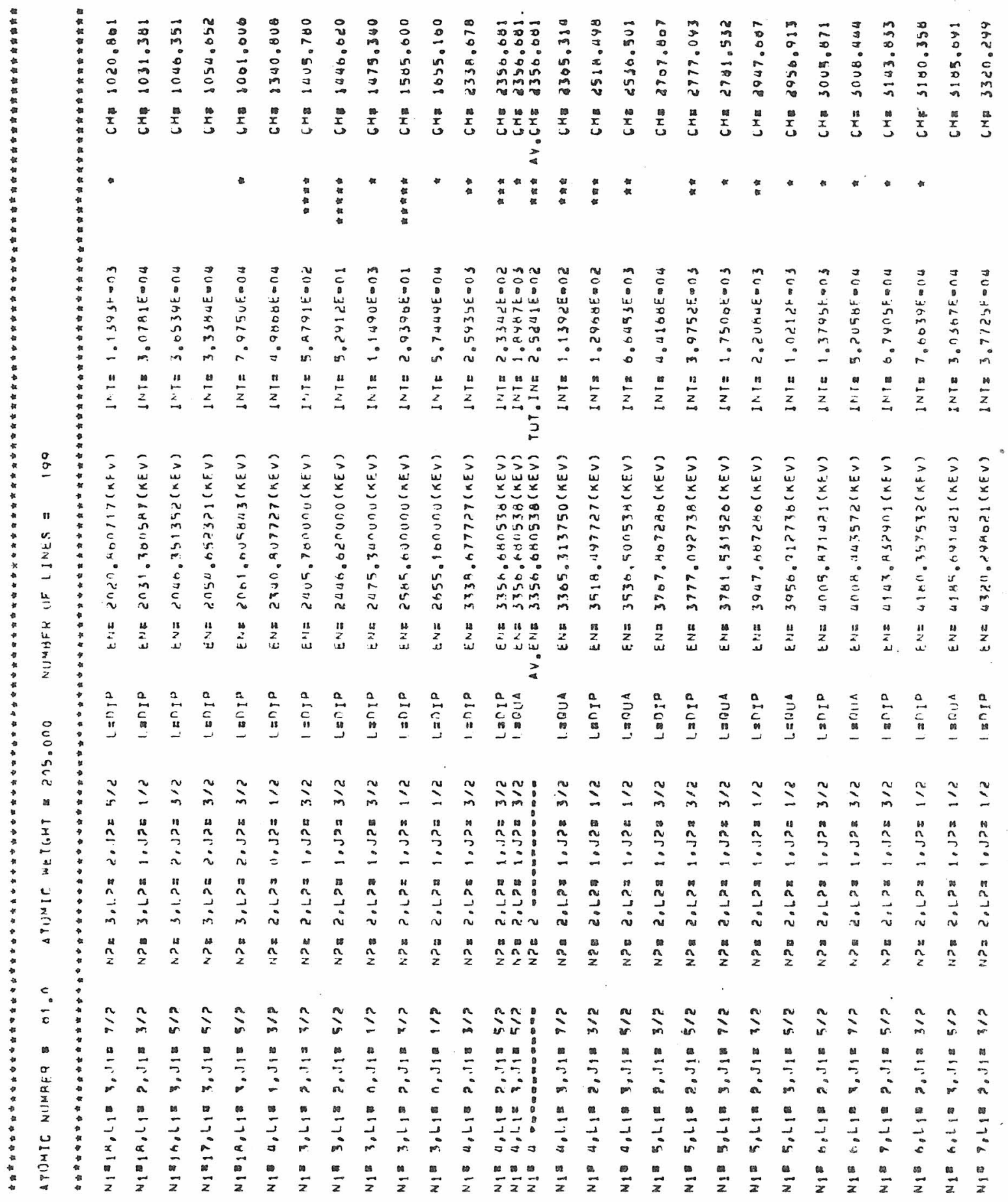




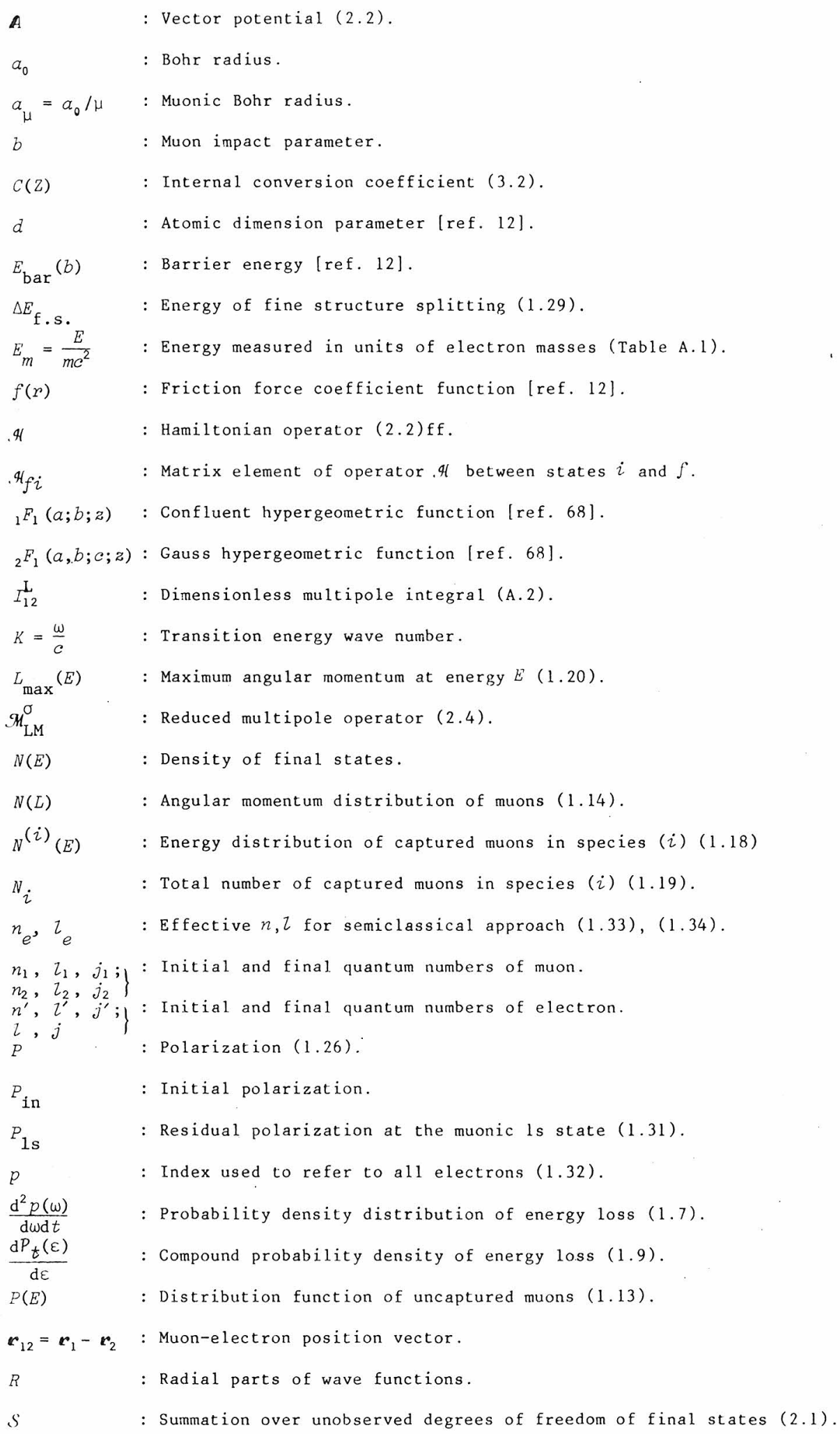




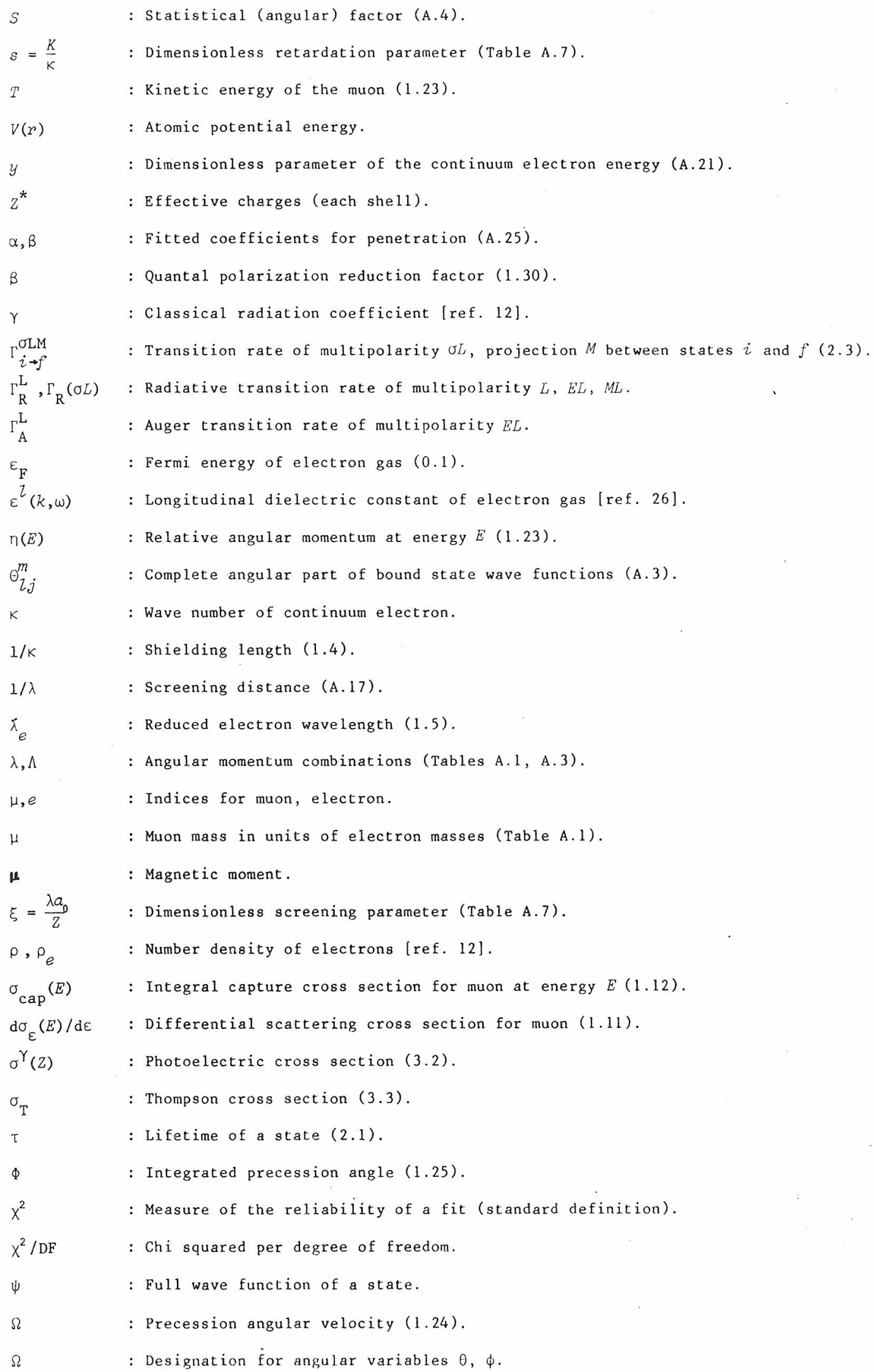




\section{$-71-$ \\ REFERENCES}

[1] U. Fano, Ann. Rev. of Nucl. Science 13 (1963) 1.

[2] C. S. Wu and L. Wilets, Ann. Rev. of Nucl. Science 19 (1969) 527.

[3] S. S. Gershtein and L. I. Ponomarev, in Mron Physics III V. W. Hughes \& C. S. Wu ed., Academic Press N. Y. 1975 p. $142 \mathrm{ff}$.

[4] J. Hüfner, F. Scheck, and C. S. Wu, ibid. vol. I p. $202 \mathrm{ff}$.

[5] V. S. Evseev, ibid. vol. III p. $236 \mathrm{ff}$.

[6] N. Bohr, Phil. Mag. 25 (1913) 10.

[7] H. A. Bethe, Ann. Physik 5 (1930) 325

[8] F. Bloch, ibid. 16 (1933) 285.

[9]. M. Leon and R. Seki, Phys. Rev. Lett. 32 (1974) 132

[10] M. Leon and R. Seki, Nucl. Phys. A282 (1977) 445.

[11] M. Leon and J. H. Miller, Nucl. Phys. A282 (1977) 461.

[12] P. Voge1, P. K. Haff, V. Akylas, and A. Winther, Nucl. Phys. A254 (1975) 445.

[13] P. Vogel, A. Winther, and V. Akylas, Phys. Lett. 70B (1977) 39.

[14] P. Vogel and V. R. Akylas, Nucl. Phys. A276 (1977) 466.

[15] J. Hïfner, Z. Phys. 195 (1966) 365.

[16] N. Barash-Schmidt et al., Particle Properties, CERN, April 1976.

[17] H. Primakoff, Rev. Mod. Phys. 31 (1959) 802.

[18] E. Fermi and E. Teller, Phys. Rev. 72 (1947) 399.

[19] V. G. Zinov, A. D. Konin, and A. J. Mukhin, Sov. J. Nucl. Phys. 2 (1965) 613.

[20] L. F. Mausner, R. A. Naumann, J. A. Monard, and S. N. Kaplan, Phys. Lett. 56B (1975) 145

[21] H. Daniel, Phys. Rev. Lett. 35 (1975) 1649.

[22] J. D. Knight et al., Phys. Rev. Al3 (1976) 43.

[23] R. Bergmann et al., Z. Physik A280 (1977) 27.

[24] J. Lindhard and M. Scharff, Mat. Fys. Medd. Dan. Vid. Selsk. 27 (1953) No. 15.

[25] J. Lindhard, ibid. 28 (1954) No. 8

[26] J. Lindhard and A. Winther, ibid. 34 (1964) No. 4.

[27] P. Gombàs, Die Statistische Theorie des Atoms, Springer Verlag, Vienna, 1949.

[28] E. Clementi and C. Roetti, Atomic Data and Nuclear Data Tables 14 (1974) 177.

[29] J. D. Jackson, CZassical Electrodynamics, 2nd ed., Wiley, New York, 1975.

[30] V. R. Akylas and P. Vogel, Hyperfine Interactions 3 (1977) 77.

[31] T. Yamazaki et al., Phys. Rev. Lett. 39 (1977) 1462.

[32] T. Yamazaki et al., Physica Scripta 11 (1975) 33.

[33] H. Überall, Phys. Rev. 114 (1959) 1640.

[34] E. Libkin, Phys. Rev. 119 (1960) 815.

[35] A. P. Bukhvostov, Sov. J. Nuc1. Phys. 9 (1969) 65

[36] I. M. Shmushkevich, Nuc1. Phys. 11 (1959) 419. 
[37] R. A. Mann and M. E. Rose, Phys. Rev. 121 (1961) 293.

[38] G. Ya. Korenmann, Yad. Fiz. 21 (1974) 772.

[39] K. Nagamine and T. Yamazaki, Nucl. Phys. A219 (1974) 104.

[40] L. M. Simons, Parity Mixtures in Muonic Atoms, preprint CERN, April 1975.

[41] G. R. Burbidge, PhD thesis, London (1951) unpublished.

[42] G. R. Burbidge and A. H. de Borde, Phys. Rev. 89 (1953) 189.

[43] A. H: de Borde, Proc. Phys. Soc. A67 (1954) 57.

[44] Y. Eisenberg and D. Kessler, Nuovo Cimento XIX (1961) 1195.

[45] M. E. Rose, Multipole Fiezds, John Wiley and Sons, N. Y: 1955.

[46] G. W. F. Drake, Phys. Rev. A3 (1977) 909.

[47] P. Martinetal., Phys. Rev. Lett. 25 (1970) 1406.

[48] H. Backe et al., Nuc1. Phys. Al89 (1972) 472.

[49] C. M. Lederer et al., Table of Isotopes, 6th ed., John Wiley and Sons, N. Y. 1967.

[50] R. S. Hager and E. C. Seltzer, Nucl. Data A4 $1 \& 2$ (1968) 1. Recent calculations of Baud et al. (ADNT) were not available during the preparation of the thesis.

[51] E. L. Church and P. Morrison, Phys. Rev. 983 (1955) 718.

[52] U. Raff et al., Nucl. Phys. A223 (1974) 429.

[53] P. Vogel, Phys. Rev. A $\underline{8}$ (1973) 2292.

[54] F. Herman and S. Skillman, Atomic Structure Calculations, Prentice-Ha11, N. J., 1963.

[55] M. H. Hebb and G. E. Uhlenbeck, Physica V 7 (1938) 605.

[56] M. H. Hebb and E. Nelson, Phys. Rev. 58 (1949) 486.

[57] R. A. Ferrel1, Phys. Rev. Lett. 4 (1960) 425.

[58] W. J. Veigele, ADNDT $\underline{5}$ (1973) 51.

[59] W. H. McMaster et al., Compilation of X-Ray Cross Sections, UCRL-50174 (1969).

[60] F. J. Hartmann et al., Phys. Rev. Lett. 37 (1976) 331.

[61] J. H. Scofield, ADNDT 14 (1974) 121 .

[62] A. Brandão-d'oliveira et al., Phys. Rev. A 135 (1976) 1772.

[63] Computer Physics Communications, CPC Program Library, Queen's University of Belfast, N. Ireland.

[64] Program MUONIC ATOM CASCADE, Jdentification Code: AAMA.

[65] S. A. Moszkowski in Alpha-, Beta- and Gamma-Ray Spectroscopy, K. Siegbahn, ed., North-Holland, Amsterdam, 1965 , p. $863 \mathrm{ff}$.

[66] A. C. Hearn, REDUCE-2, University of Utah, 2nd ed., March 1973.

[67] C. Kittel, Introduction to Solid State Physics, 3d ed., John Wiley and Sons, N. Y. 1967.

[68] W. Magnus et al., Formizas and Theorems for the Special Functions of Mathematical Analysis, Springer-Verlag, N.Y. 1966. 